\title{
Constitutive Modeling of Metastable Austenitic Stainless Steel
}

E.S. Perdahcioğlu 
This work is part of the research program of the 'Stichting voor Fundamenteel Onderzoek der Materie (FOM)', which is financially supported by the 'Nederlandse Organisatie voor Wetenschappelijk Onderzoek (NWO)'. This research was carried out under projectnumber 02EMM30-2 in the framework of the Strategic Research program of the Materials Innovation Institute (M2i).

Samenstelling van de promotiecommissie:

voorzitter en secretaris:

Prof. dr. F. Eising

Universiteit Twente

promotor:

Prof. dr. ir. J. Huétink

Universiteit Twente

assistent promotor:

Dr. ir. H.J.M. Geijselaers

Universiteit Twente

leden:

Prof. dr. ir. R. Akkerman

Universiteit Twente

Prof. dr. ing. W. Bleck

RWTH Aachen University

Dr. A.J. Böttger

Technische Universiteit Delft

Prof. dr. ir. M.G.D. Geers

Technische Universiteit Eindhoven

Prof. dr. rer. -nat S. Luding

Universiteit Twente

Dr. ir. A.S.J. Suiker

Technische Universiteit Delft

ISBN 978-90-365-2769-9

1st printing December 2008

Keywords: TRIP, martensite, phase transformation, constitutive modeling

This thesis was prepared with LATEX by the author and printed by PrintPartners Ipskamp, Enschede, from an electronic document.

Copyright (c) 2008 by E.S. Perdahcioğlu, Enschede, The Netherlands

All rights reserved. No part of this publication may be reproduced, stored in a retrieval system, or transmitted in any form or by any means, electronic, mechanical, photocopying, recording or otherwise, without prior written permission of the copyright holder. 


\section{CONSTITUTIVE MODELING OF METASTABLE AUSTENITIC STAINLESS STEEL}

\section{PROEFSCHRIFT}

ter verkrijging van

de graad van doctor aan de Universiteit Twente, op gezag van de rector magnificus, prof.dr. W.H.M. Zijm, volgens besluit van het College voor Promoties in het openbaar te verdedigen op woensdag 17 december 2008 om 13.15 uur

door

Emin Semih Perdahcıŏglu

geboren op 29 juli 1977

te Bursa, Turkije 
Dit proefschrift is goedgekeurd door de promotor:

Prof. dr. ir. J. Huétink

en de assistent promotor:

Dr. ir. H.J.M. Geijselaers 


\section{Summary}

Metastable austenitic stainless steels combine high formability and high strength, which are generally opposing properties in materials. This property is a consequence of the martensitic phase transformation that takes place during deformation. This transformation is purely mechanically induced although temperature has a very strong influence on the kinetics of the process. As the temperature decreases, the transformation rate increases since martensite becomes more stable relative to austenite.

These materials are currently used in industry, for instance in household appliances, although the manufacturing processes for these products are difficult to optimize. This is due to the absence of material models that can adequately describe the complex mechanical behavior of these steels. Although it is possible to find phenomenological constitutive models for this class of materials in the literature, obtaining parameters for these models requires extensive mechanical testing. The main goal of this study is to develop a constitutive model that incorporates a physically based description of the phase transformation phenomenon.

To understand the physics of the mechanically induced transformation, first, mechanical tests have been carried out. The experiments were aimed at determining the effects of stress state and plastic strain on the transformation behavior. The results pronounced the effect of stress over that of plastic strain suggesting that the transformation could be explained by a stress-based model.

The crystallography of martensitic transformations has been studied and with a simple model in mesoscale the mechanical driving force concept was exploited. Based on the results of the model, a physical explanation for the transformation was proposed and verified with experiments. A stress based transformation criterion was proposed which was demonstrated to agree very well with experimental results. Furthermore, a continuum level expression for the martensite volume fraction as a function of the applied stress was proposed.

Computing the mechanical behavior of the material during transformation requires taking into account the individual behavior of the austenite and martensite phases as well as their mutual interaction. To serve this purpose, a mean-field homogenization model was utilized. Different algorithms found in the literature were tested and two new algorithms were proposed. These were demonstrated to be reliable as well as computationally efficient. 
A constitutive model was proposed that is based on a combination of the transformation model and the homogenization model. In addition to these, improvements were made to incorporate the effects of evolving volume fractions of the phases as well as the transformation plasticity phenomenon. The results of the algorithm were compared to the experimental results and a good agreement was obtained. The results prove that the stress state and temperature dependent transformation behavior can be described by the stress-driven transformation model accurately, with only a small number of physical parameters. 


\section{Samenvatting}

Metastabiel austenitisch roestvast staal combineert hoge vervormbaarheid met hoge sterkte, wat over het algemeen tegengestelde eigenschappen zijn. Dit gedrag is een direct gevolg van de austeniet-martensiet fasetransformatie die plaats vindt gedurende deformatie. Deze transformatie wordt zuiver mechanisch geactiveerd, hoewel de temperatuur een sterke invloed heeft op de kinetica van het proces. Met afnemende temperatuur neemt de transformatiesnelheid toe omdat martensiet relatief stabieler wordt ten op zichte van austeniet.

Deze staalsoort wordt al toegepast in de industrie, bijvoorbeeld in huishoudelijke toepassingen, hoewel het fabricageproces voor deze producten moeilijk te optimaliseren is. De oorzaak hiervan ligt in het gebrek aan materiaalmodellen die het complexe mechanische gedrag van dit staal kunnen beschrijven. Hoewel fenomenologische constitutieve modellen gevonden kunnen worden in de literatuur, vereist het bepalen van de materiaalparameters voor deze modellen een uitgebreide testprocedure. Het hoofddoel van dit onderzoek is het ontwikkelen van een constitutief model dat een fysisch gebaseerde beschrijving van de fasetransformatie bevat.

Om de fysica achter de mechanisch geactiveerde transformatie te achterhalen zijn ten eerste mechanische testen uitgevoerd. De experimenten zijn bedoeld om de effecten van de spanningstoestand en de plastische rek op de transformatie te bepalen. Uit de resultaten blijkt dat de invloed van spanning groter is dan van de plastische rek. Dit gaf aanleiding om de transformatie te beschrijven met een model gebaseerd op spanningen.

De kristallografie van de martensietvorming is bestudeerd en een simpel model in de meso-schaal beschrijft het concept van de mechanische activeringskracht. Gebaseerd op de resultaten van het model is een fysische verklaring van de transformatie voorgesteld, welke is geverifieerd met experimenten. Een transformatiecriterium gebaseerd op spanning is geïntroduceerd en de resultaten hiervan komen goed overeen met de experimenten. Bovendien is voor het macroniveau een uitdrukking voorgesteld voor de martensietfractie als functie van de aangebrachte spanning.

Het berekenen van het mechanisch gedrag van het materiaal gedurende transformatie vereist dat zowel met het individuele gedrag van de austeniet- en martensietfase, als wel hun onderlinge relatie, rekening gehouden moet worden. Daarom wordt een homogenisatie gebruikt die de spanningen en rekken over verschillende velden middelt. Verschillende algoritmen uit de literatuur zijn getest en twee nieuwe algoritmen wordt 
voorgesteld. Er wordt aangetoond dat deze algoritmen zowel betrouwbaar als efficiënt zijn.

Gebaseerd op de combinatie van het transformatie- en homogenisatiemodel wordt een constitutief model voorgesteld. Bovendien zijn verbeteringen aangebracht om zowel het transformatie-plasticiteitverschijnsel als wel de effecten van veranderende volumefracties van de verschillende fases op te nemen. De resultaten van dit algoritme en de experimenten komen goed overeen. Het spanningsgestuurde transformatiemodel laat zien dat het transformatiegedrag, afhankelijk van de spanningstoestand en de temperatuur, nauwkeurig beschreven kan worden. Slecht een klein aantal fysische parameters is nodig. 


\section{Contents}

Summary v v v v v v

Samenvatting vii

1 Introduction $\quad 1$

1.1 About this thesis . . . . . . . . . . . . . . . 2

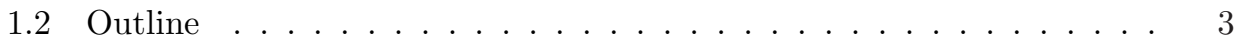

1.3 Notation . . . . . . . . . . . . . . . . . . . . 4

2 Mechanically induced martensitic transformation 5

2.1 Martensitic transformation . . . . . . . . . . . . . 5

2.2 Crystallography of martensitic transformation . . . . . . . . . . . . . . 9

2.3 Mechanically induced transformation . . . . . . . . . . . . . . 14

2.3.1 Athermal transformation under stress . . . . . . . . . . . . 15

2.3.2 Deformation-induced transformation . . . . . . . . . . . . 17

2.4 TRansformation Induced Plasticity (TRIP) . . . . . . . . . . . . . . 23

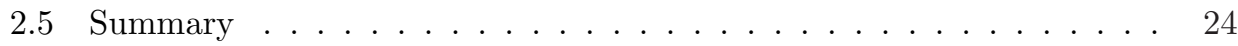

3 Experiments $\quad 27$

3.1 Material . . . . . . . . . . . . . . . . . . . . . 27

3.2 Magnetic sensor . . . . . . . . . . . . . . . . . . . 28

3.3 Effect of stress on transformation kinetics . . . . . . . . . . . . . . . 28

3.3.1 Biaxial tests . . . . . . . . . . . . . . . . . 29

3.3 .2 Stress state and strain . . . . . . . . . . . . . . . . . . 30

3.3.3 Tests .............................. 31

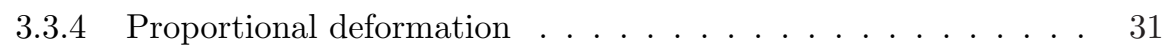

3.3.5 Non-proportional deformation . . . . . . . . . . . . . . 34

3.3.6 Reproducibility . . . . . . . . . . . . . . . . 36

3.4 Effect of strain on transformation kinetics . . . . . . . . . . . . . . . 37

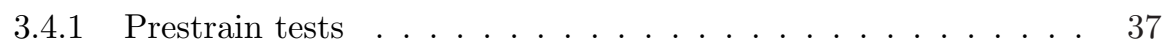

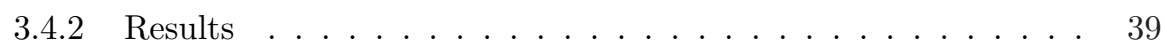

3.5 Summary and conclusions . . . . . . . . . . . . . . . . . . . 42 
4 Transformation model $\quad 43$

4.1 Introduction . . . . . . . . . . . . . . . . . . . . . . . . . . . . . . . . . 43

4.2 Mechanical Driving Force . . . . . . . . . . . . . . . . . . 44

4.3 Transformation criterion . . . . . . . . . . . . . 46

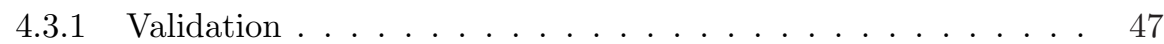

4.4 Evolution of martensite fraction . . . . . . . . . . . . . . . . . . . . . . . . . . . 49

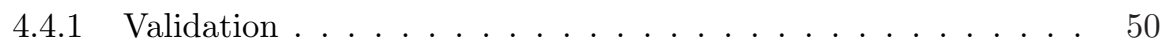

4.4.2 Phenomenological extension . . . . . . . . . . . . 53

4.5 Transformation plasticity . . . . . . . . . . . . . . . 54

4.6 Summary and conclusions . . . . . . . . . . . . . 56

5 Homogenization of elastic-plastic composites $\quad \mathbf{5 7}$

5.1 General concepts in homogenization . . . . . . . . . . . . . 58

5.1 .1 Scale transition . . . . . . . . . . . . . . . 59

5.1 .2 Hill's lemma . . . . . . . . . . . . . . . . 59

5.2 Homogenization of elastic composites . . . . . . . . . . . . . . 59

5.2.1 Strain and Stress concentration . . . . . . . . . . . . . 60

5.2.2 Homogenized elastic response . . . . . . . . . . . . . . . 61

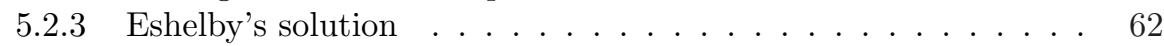

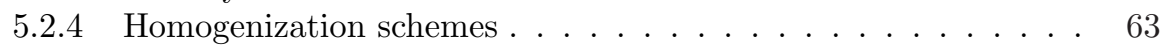

5.2.5 Comparison of models . . . . . . . . . . . . . . 68

5.3 Homogenization of elastic-plastic composites . . . . . . . . . . . . . 70

5.3.1 Large deformations . . . . . . . . . . . . . . . . . . 71

5.3.2 Linearization of elastic-plastic response . . . . . . . . . . . 72

5.3.3 Isotropic projection of the tangent moduli . . . . . . . . . . . . 73

5.3 .4 Solution algorithm . . . . . . . . . . . . . . . . 74

5.3 .5 Results and discussion . . . . . . . . . . . . . . . . . . . . 74

5.4 Summary and conclusions . . . . . . . . . . . . . . . 82

6 Constitutive model $\quad \mathbf{8 5}$

6.1 General concepts in constitutive modeling . . . . . . . . . . . . . . 85

6.2 Decomposition of strain . . . . . . . . . . . . . . . . 87

6.3 Single phase constitutive model . . . . . . . . . . . . . . . . 88

6.3.1 Hypoelastic-plastic constitutive model . . . . . . . . . . . . 89

6.3 .2 J2-Flow plasticity . . . . . . . . . . . . . . . . . . . . . . . 90

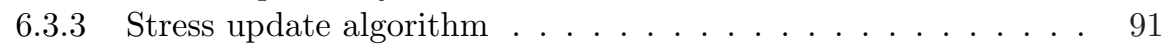

6.4 Transformation . . . . . . . . . . . . . . . . . 93

6.5 Stress integration and material tangent . . . . . . . . . . . . 96

6.6 Results . . . . . . . . . . . . . . . . . . . . 97

6.7 Summary and conclusions . . . . . . . . . . . . . . . . 101

7 Application 103

7.1 Bending simulation . . . . . . . . . . . . . . . . . 103

7.2 Results . . . . . . . . . . . . . . . . . . . . . . 104

7.3 Summary and conclusions . . . . . . . . . . . . . . . . 109

8 Conclusions and Recommendations $\quad 111$ 
A Expressions for the Eshelby tensor 115

B Fixed Point Iteration and Broyden methods 117

C Calibration of the magnetic sensor 119

Nomenclature 121

Acknowledgments 125

References 127 



\section{Introduction}

'As a blacksmith plunges an axe or hatchet into cold water to temper it -for it is this that gives strength to the iron- and it makes a great hiss as he does so, even thus did the Cyclops' eye hiss round the beam of olive wood, and his hideous yells made the cave ring again.', from The Odyssey by Homer, 800 B.C. [35].

For millennia, it has been known that steel gains strength and hardness upon quenching. The physics of this phenomenon on the other hand, was only discovered in the late $19^{\text {th }}$ century with the help of optical microscopes that allowed researchers to observe the microstructure of metals [79]. From these observations it is known that metals are comprised of different phases, defined as portions of the alloy that have uniform physical and chemical characteristics. The stability of a phase at a given temperature is dictated by thermodynamics. During cooling and heating, due to the change in the relative stabilities of phases, phase transformations take place. These transformations require atoms to rearrange in the crystal structure to form the new phase. If the rearrangement of atoms is inhibited by external factors, the transformation can be suppressed which results in a phase existing in a metastable state.

One of the phases of steel found at temperatures above the eutectoid point is austenite. The phenomenon that takes place upon quenching is the phase transformation between the phases austenite and martensite. In metastable austenitic steels, due to the presence of alloying elements such as Nickel, austenite is retained upon cooling to room temperature.

Steel is the most commonly used metal in industry, with the applications ranging from car parts to medical tools, buildings to household appliances. Steel is very attractive because of the low price and the variety of mechanical properties it offers. By modification of the alloying elements and the heat treatment, it is possible to have on the one hand a very soft and ductile material and on the other, a very hard and brittle one. A steel with high strength is very attractive, since it allows in many circumstances less material to be used for the same application, in other words: weight 
reduction. The negative side however is that a material with higher strength implies a material that is more difficult to form into a product.

Metastable austenitic stainless steels are exceptional materials in this sense because although they have high strength, they offer high formability. This property comes from the fact that the steel undergoes a phase transformation during the forming operation. In this case, the transformation is not driven by a change in temperature; it is caused by the deformation process. Although the mechanical properties make the material very attractive, it requires a lot of knowledge and expertise to control the transformation to have the desired product properties after forming.

As the materials and product designs get more complex, the role of numerical simulations in the manufacturing process becomes more important. There have been significant improvements in the numerical methods used for simulating forming operations, such as the Finite Element method, in recent years. However, the accuracy of these methods is usually limited by the models that describe the material behavior. These models are referred to as constitutive models, and define the response of a material to a given deformation. Constitutive modeling can be simply described as casting knowledge of a material into a mathematical model.

\subsection{About this thesis}

The main objective of this thesis is to develop a constitutive model for metastable austenitic stainless steels. Material models for these steels can currently be found in the literature, for instance in $[74,80]$. However, experience has shown that in practice these models are not very efficient. They require excessive experimental data in simulating a certain forming operation which causes serious problems when a new process has to be designed or the material properties have to be changed. The missing ingredient in these models is believed to be an accurate model to describe the phase transformation.

Therefore in this research, considerable effort has been put in investigating experimentally and theoretically, the mechanically induced martensitic transformation phenomenon. In literature, a lot of research has been found on this matter in which, to explain the phenomenon, different theories have been proposed. However, there are apparent contradictions in the proposed theories; hence, one of the focal points of this study is to investigate the validity of these theories. The results of this study are used to develop a transformation model which takes into account the effects of temperature, plastic strain and stress state. These factors become critical in the simulation of forming operations; for example, the effect of stress state is pronounced in sheet-metal forming since different portions on the blank undergo different stressstates throughout the process.

Moreover, to have a constitutive model one needs to define (at least) the relationship between the prescribed deformation and the stress response. This is a challenging task for the case of austenitic stainless steels since the microstructure, and hence the strength of the material, evolves with deformation. Although the mechanical behavior 
of the constituent phases is known, the properties of the mixture have to be modeled by considering the mixture of phases and their mutual interaction. Consequently, a considerable part of the research has been focused on modeling the mechanical behavior of elastic-plastic composite materials.

\subsection{Outline}

A background on martensitic transformation is given in Chapter 2 along with the history and the current state of research on the mechanically induced martensitic transformation phenomenon. Experimental findings and the currently accepted theories are discussed after which a thorough analysis of the crsytallography of the martensitic transformation is presented.

In Chapter 3 the experiments that were performed on the material are discussed. Two types of tests were carried out which help to understand the effects of stress-state and plastic strain on the transformation. The procedures and the results of the tests are presented to be followed by a discussion on the implications of the results.

Having gathered experimental knowledge on the material, a transformation model was developed which is presented in Chapter 4. The model is based on the concept of a mechanical driving force and therefore, the chapter starts with a definition and computation methods of this entity. Then, a simple mesoscale model is formulated which was constructed to describe the distribution of the mechanical driving force in a polycrystalline material. Finally, the proposed phenomenological extension of this model to macroscale is introduced.

Chapter 5 deals with the computation of the stress-strain response of a material with two elastic-plastic phases. The mean-field homogenization method is described and the Eshelby theories that are employed in the procedures are summarized. This is followed by a discussion on different algorithms that are used in the homogenization study such as the Self-Consistent and Mori-Tanaka schemes. The results of the model are compared to Direct Finite Element method results found in the literature. Finally, the behavior the austenite-martensite composite for a static mixture of the phases with different volume fractions is presented.

In Chapter 6 the constructed constitutive model, that merges the transformation and the homogenization algorithms, is introduced. First, the rate form of the transformation function that has been introduced in Chapter 4 is obtained. Then, the effects of the evolving volume fraction of the phases are discussed. The details of the stress-update algorithm are given and the derivations of the material tangent are supplied. Finally, results of the algorithm are compared to experiments at an integration point level.

In Chapter 7, the results of the constitutive model in the structure level are shown. The model is implemented in the in-house implicit FE code DiekA, and simulations were performed to investigate the predictions of the model under different conditions. 


\subsection{Notation}

In the thesis, $1^{\text {st }}$ order tensors and vectors are shown in bold face and lowercase letters (a), $2^{\text {nd }}$ order tensors and matrices in bold face and uppercase letters $(\mathbf{A})$ and $4^{\text {th }}$ order tensors with caligraphic letters $(\mathcal{A})$. The components of tensor with respect to a specific basis is denoted with square brackets $([\mathbf{A}])$. The single contraction of tensors is represented by a $\operatorname{dot}\left(\mathbf{A} \cdot \mathbf{b}=A_{i j} b_{j}\right)$, double contraction by a colon $\left(\mathbf{A}: \mathbf{B}=A_{i j} B_{i j}\right)$ and quadruple contraction with two colons $\left(\mathcal{A}:: \mathcal{B}=A_{i j k l} B_{i j k l}\right)$. The dyadic (tensor) product is represented with the $\otimes \operatorname{sign}\left(\mathbf{C}=\mathbf{a} \otimes \mathbf{b}, C_{i j}=a_{i} b_{j}\right)$. 


\section{Mechanically induced martensitic transformation}

This chapter focuses on the general aspects of mechanically induced martensitic transformations, history and the currently accepted theories. Although mechanically induced, this type of transformation is essentially not very different from the other types of martensitic transformation. Consequently, in the first section the martensitic transformation theory in general will be discussed and after that the crystallography of martensitic transformations will be explained. The chapter will continue with a discussion on mechanical effects on the transformation, the experimental evidence and the theories proposed to unearth the physics behind the phenomenon.

\subsection{Martensitic transformation}

One of the most important properties of steel is its hardenability. It has been known for millennia that rapidly cooling steel from temperatures above $1000{ }^{\circ} \mathrm{C}$, i.e. water quenching, will increase its hardness significantly [35]. On the other hand, if cooling is performed slowly, i.e. inside the furnace, the resulting steel will not be as hard. Hence, it is clear that time plays an important role in the phase transformations of steel.

Figure 2.1 shows the equilibrium Fe-C phase diagram ${ }^{1}$. On this diagram every phase transformation that occurs will be reversible, meaning that the reactions take place continuously with respect to temperature and at any moment can be stopped and reversed. For this condition to be satisfied, heating and cooling must be carried out very slowly to provide the atoms with enough time to diffuse during the phase changes. Since this is an equilibrium condition, all the phases that are denoted on the diagram are equilibrium phases.

\footnotetext{
${ }^{1}$ Although $\mathrm{C}$ is more stable than $\mathrm{Fe}_{3} \mathrm{C}$, due to the extremely slow kinetics of the reaction, the diagram will be regarded as an equilibrium phase diagram.
} 


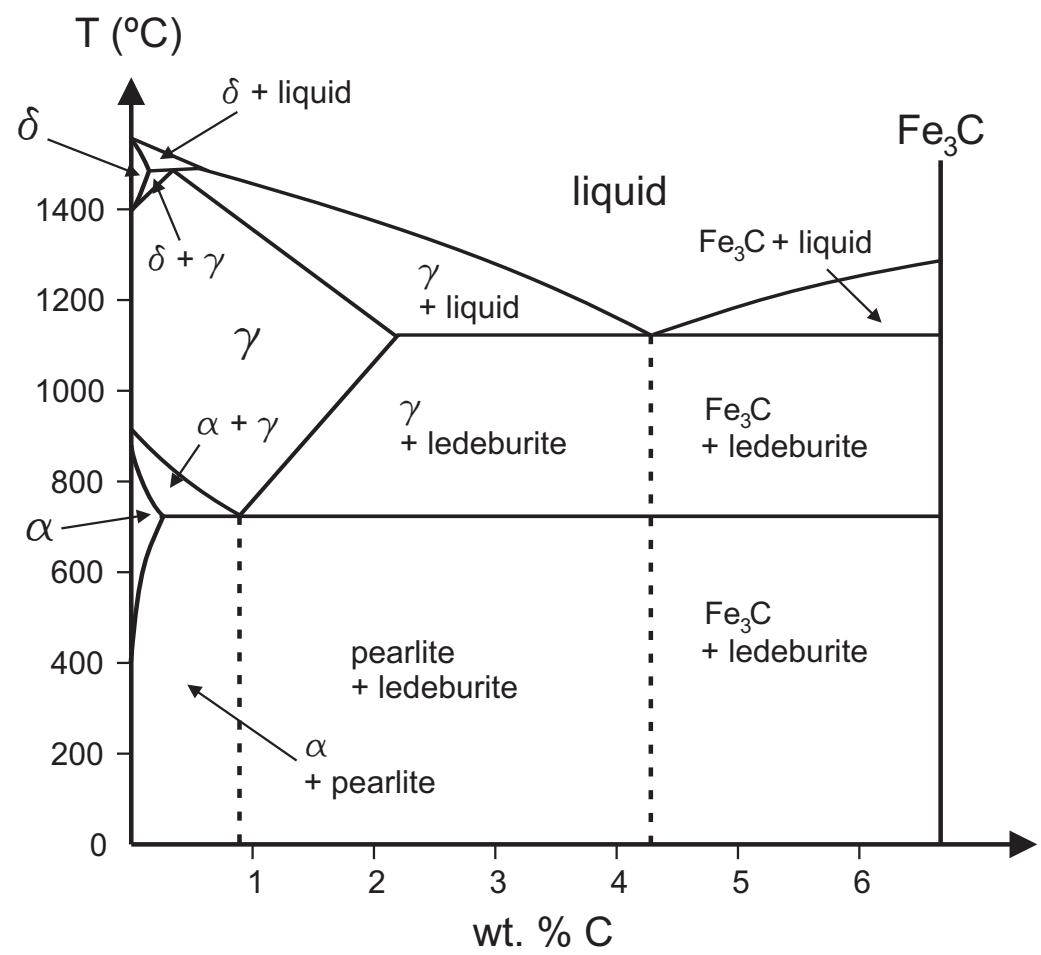

Figure 2.1: Iron - Carbon equilibrium phase diagram.

In practical circumstances however, equilibrium during cooling and heating can hardly be attained. Since the cooling rate is usually not as low as required for equilibrium, the atoms cannot diffuse to the full extent, hence resulting in the formation of non-equilibrium phases. Non-equilibrium phase transformations are generally not reversible, meaning that during the transformation it might not be possible to stop or reverse $\mathrm{it}^{2}$. The time dimension has to be introduced to describe the phase transformation and hence, an equilibrium phase diagram is no longer sufficient. Instead, Time-Temperature-Transformation (TTT) or ContinuousCooling-Transformation (CCT) diagrams are needed. The TTT diagram of a plaincarbon steel with eutectoid composition is schematically illustrated in Figure 2.2. Pearlite occurs after a diffusional transformation which transforms austenite to a mixture of ferrite and cementite. Diffusion is much less pronounced in the formation of bainite leading to the formation of cementite particles in fine aggregates of ferrite plates. The $C$ shape of the curves can be credited to the opposite relation of on the one hand chemical driving force and on the other diffusivity to temperature. At high temperatures diffusion is easy but the driving force is small, whereas at low temperatures diffusion becomes less easy although the driving force increases. At intermediate temperatures, since both diffusion and the driving force are favored,

\footnotetext{
${ }^{2}$ Reversibility in thermodynamics is defined as a reaction being continuous and differentiable in time or temperature.
} 


\section{$\mathrm{T}\left({ }^{\circ} \mathrm{C}\right)$}

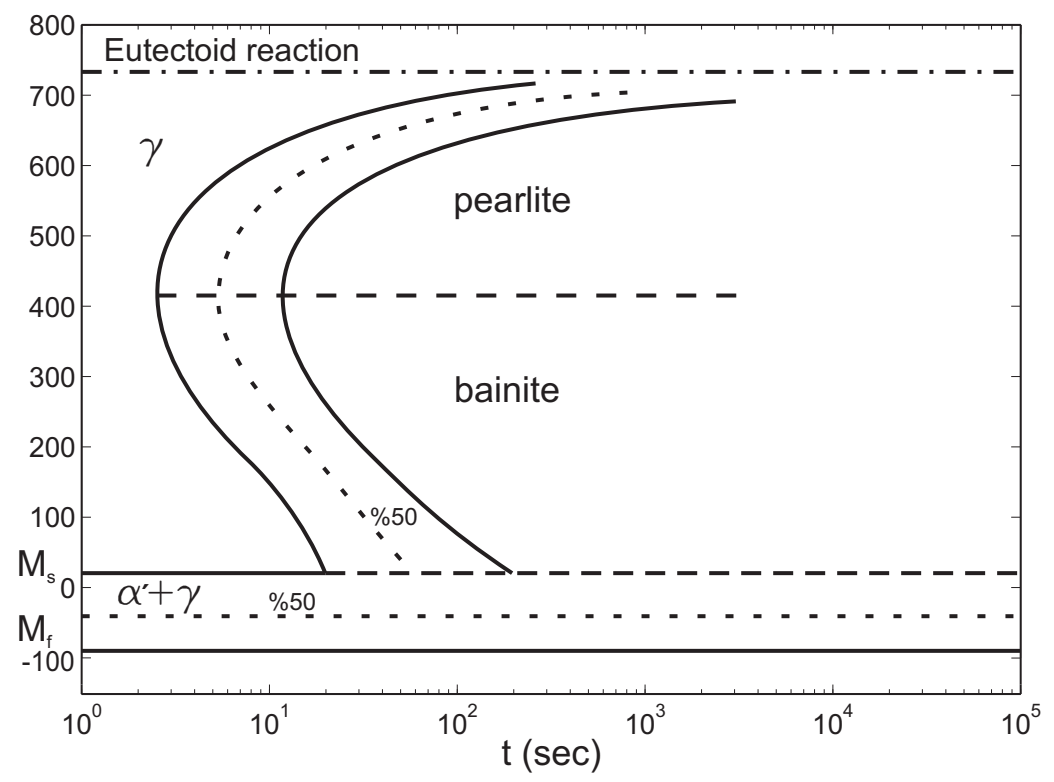

Figure 2.2: Schematic representation of the TTT (time-temperature-transformation) diagram of a plain-carbon steel.

transformation proceeds rapidly.

In most steels, martensitic transformation takes place between austenite, $\gamma$, and martensite, $\alpha^{\prime}$, phases. Steel, in its austenitic state, must be quenched below a certain temperature very rapidly for this reaction to start. The specific temperature below which the reaction takes place is called the martensite start, $M_{\mathrm{s}}$, temperature. The reason of this transformation is that for the given chemical composition of steel at low temperatures austenite is no longer the stable phase. The equilibrium stable phase is ferrite, $\alpha$, but in the absence of diffusion this transformation is not feasible. Therefore, the atoms find an escape route and form a structure that resembles ferrite by only shifting slightly from their lattice locations. For this reason martensitic transformations are alternatively referred to as diffusionless (or displacive) transformations [66]. The free energy curves of the phases austenite and martensite are illustrated schematically in Figure 2.3 [42]. It is seen that martensite is more stable than austenite at temperatures below $T_{0}$ however, the martensitic transformation is only observed below the $M_{\mathrm{s}}$ temperature. This is because the transformation requires additional energy for new surfaces to be created and the surrounding matrix to be elastically distorted. The latter is due to the difference in densities of the austenite and martensite phases. The combination of all opposing effects can be thought of as an energy barrier that needs to be overcome for the transformation to start.

The free energy difference is generally referred to as the chemical driving force, inherently describing the fact that this difference is the reason for the transformation 
and is a consequence of the chemical composition only. For Iron-Carbon alloys, the free energy difference at $M_{\mathrm{S}}$ is of the order of $1200 \mathrm{~J} / \mathrm{mole}$ [70].

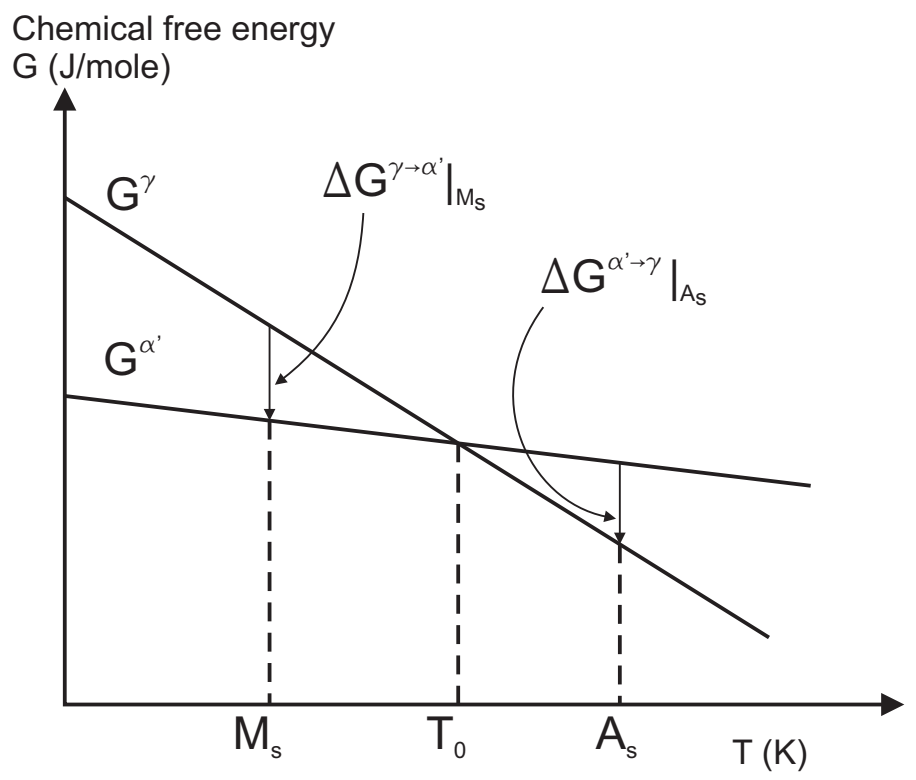

Figure 2.3: Schematic representation of the free energy curves of austenite $\gamma$ and martensite $\alpha^{\prime}$ versus temperature for an iron base alloy.

The most prominent difference between the two types of transformations is the way the atoms rearrange in the crystal lattice. In diffusional transformations atoms exchange locations between neighbors whereas in diffusionless ones they do not. Instead, they move in clusters and the average relative displacement of each atom is less than one lattice spacing. Due to the fact that there is no diffusion involved, the transformation can proceed very rapidly, of the order of speed of sound in solids, and the chemical composition of the martensite will be the same as the parent austenite.

It is widely accepted that martensite nucleates in the austenite matrix heterogeneously [53], meaning that there must be nucleation sites, or embryos, on which transformation can take place.

Martensite in steel has a lower density compared to austenite. Hence, the martensitic transformation is always accompanied by a volume change. This leads to shape distortions and residual stresses and even cracking after quenching. Furthermore, it has a $\mathrm{BCT}^{3}$ type crystal lattice compared to an $\mathrm{FCC}^{4}$ type austenitic lattice. The tetragonality is a consequence of the $\mathrm{C}$ atoms to be placed at biased locations in the crystal lattice. As the $\mathrm{C}$ concentration gets lower the tetragonality disappears making the lattice cubic.

\footnotetext{
${ }^{3}$ Body centered tetragonal

${ }^{4}$ Face centered cubic
} 
Quenching transforms the austenite phase into martensite to an extent determined by the temperature of quenching. It can be seen on the TTT diagram (see Figure 2.2) that the lower the quenching temperature, the more martensite forms. The remaining austenite in the crystal is referred to as retained austenite. Since the stable phase at the quenching temperature is martensite, the retained austenite phase is in a metastable state.

The type of transformation where only the temperature controls the amount transformed is called athermal transformation. The term is a result of the fact that time has no role in transformation kinetics and hence there is no thermal activation ${ }^{5}$.

Martensite is observed to form from austenite under three different types of transformation mechanisms: athermal (as mentioned earlier), isothermal and burst [75]. Isothermal transformation takes place at a constant temperature as a function of time (thermally activated). When the martensite fraction is plotted as a function of time, the resulting curves are of a sigmoid shape, i.e. the transformation rate is initially slow but after a few percent of transformation it increases by around one order of magnitude and then slowly decreases. This behavior is associated with a phenomenon called autocatalysis, meaning that transformation itself triggers more transformation.

In the burst mode, quenching to a certain temperature, an abrupt burst of up to around $50 \%$ martensite formation is observed which is followed by a subsequent athermal or isothermal mode of transformation.

\subsection{Crystallography of martensitic transformation}

In this section the crystallographic details of the martensitic transformation will be presented. The specific way that the atoms rearrange during transformation results in the formation of invariant planes, i.e. planes that are not distorted nor rotated during transformation. These planes are generally termed habit planes. On these planes a deformation takes place, a combination of dilatation and shear, which will be referred to as the transformation deformation. It has been shown by Wechsler, et al. (WLR) [90] as well as Bowles and Mackenzie [10-12] that it is possible to calculate the transformation deformation, the habit plane and shear directions if the lattice parameters of the phases are known. The WLR and Bowles-Mackenzie methods have been evaluated in more detail and compared by Bhadeshia [6] and Wayman [89], who found also that the two methods are essentially equivalent. Therefore in essence, these methods are different solution approaches to the same theory. Due to the fact that both methods rely on the observation that the transformation leaves an undistorted plane at the austenite-martensite interface, this theory is usually referred to as the phenomenological theory of martensitic transformation.

Martensite, as already mentioned has a $\mathrm{BCC}^{6}$ type crystal structure, whereas

\footnotetext{
${ }^{5}$ Thermal activation is a general term in materials science implying the effect of constant temperature on activating a mechanism, such as nucleation.

${ }^{6}$ The steel is assumed to have a low carbon concentration.
} 
austenite has an FCC type. The unit cells of these types of crystals are illustrated in Figure 2.4.
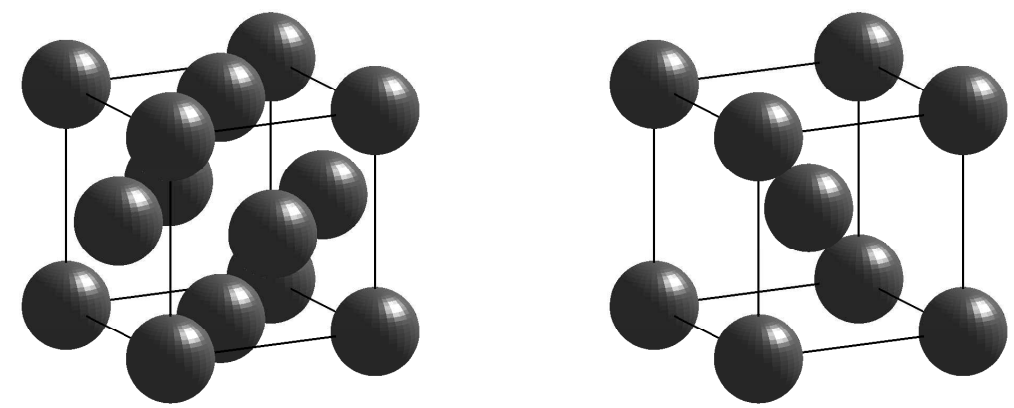

Figure 2.4: Illustration of the FCC (left) and BCC (right) type unit cells.

Transformation from austenite to martensite requires a redistribution of atoms in the FCC lattice to form the BCC lattice. One of the theories that describe this transition is given by Bain which proposes the transition to be the one that requires minimum displacement of the atoms. This distortion can be visualized with the atoms that already have a BCT type structure within the FCC lattice, as shown in Figure 2.5.
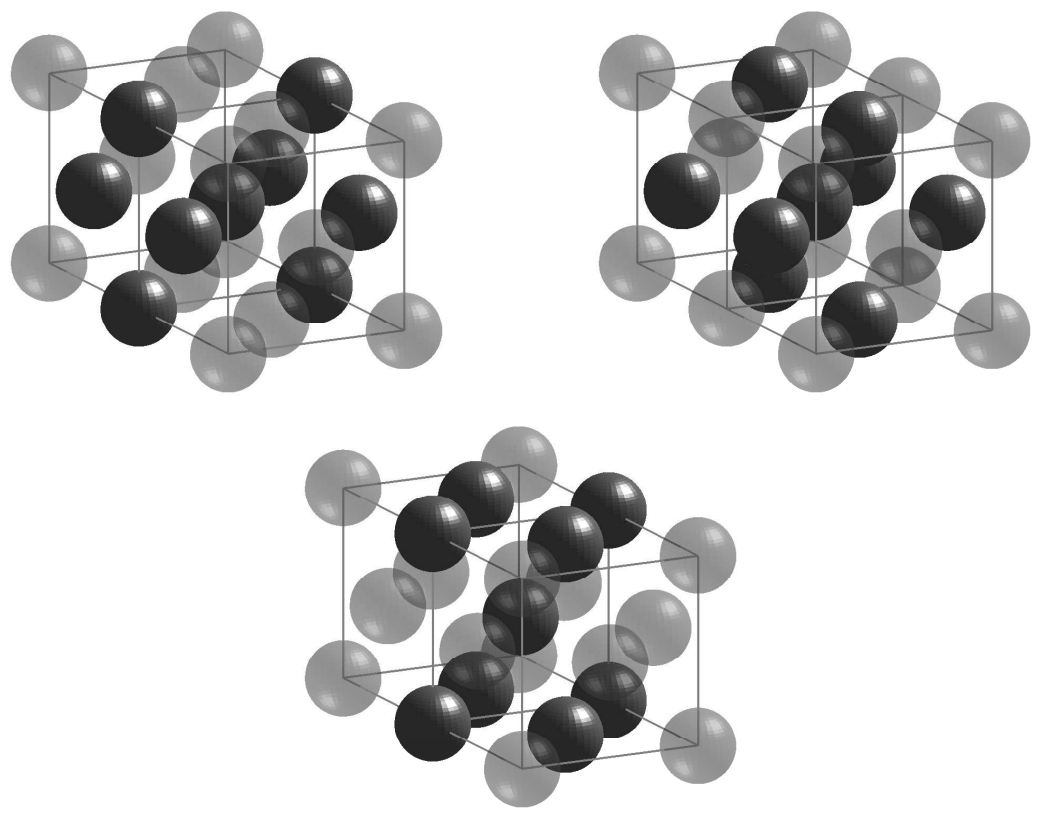

Figure 2.5: Visualization of the BCT type structure in the FCC lattice.

The transition therefore, requires the long axis to contract and the perpendicular axes to expand such that all axes have equal length and a form a cubic lattice. Of 
course, due to symmetry of the lattice, the long axis and the perpendicular ones are interchangeable. The deformation can be expressed by one of the following tensors, with components given with respect to the austenite lattice:

$$
\left[\mathbf{B}_{1}\right]=\left[\begin{array}{ccc}
\eta_{2} & 0 & 0 \\
0 & \eta_{1} & 0 \\
0 & 0 & \eta_{1}
\end{array}\right], \quad\left[\mathbf{B}_{2}\right]=\left[\begin{array}{ccc}
\eta_{1} & 0 & 0 \\
0 & \eta_{2} & 0 \\
0 & 0 & \eta_{1}
\end{array}\right], \quad\left[\mathbf{B}_{3}\right]=\left[\begin{array}{ccc}
\eta_{1} & 0 & 0 \\
0 & \eta_{1} & 0 \\
0 & 0 & \eta_{2}
\end{array}\right]
$$

where

$$
\eta_{1}=\frac{\sqrt{2} a}{a_{0}}, \quad \eta_{2}=\frac{a}{a_{0}}
$$

and $a_{0}$ is the lattice parameter of austenite, $\gamma$, and $a$ is that of martensite, $\alpha^{\prime}$.

However, the Bain distortion is not sufficient to describe the complete transformation. As a start, the deformation tensors given in Equation (2.1) leave the lattice incoherent, i.e. the atomic positions in the adjoining planes do not coincide. Coupled with a rotation, however, there exists a line which is neither distorted nor rotated, making the deformation an invariant-line strain. This can be visualized in Figure 2.6 where the deformation is shown on the $z=0$ plane. In 3-D space however, the invariant-lines form a cone due to rotational symmetry.
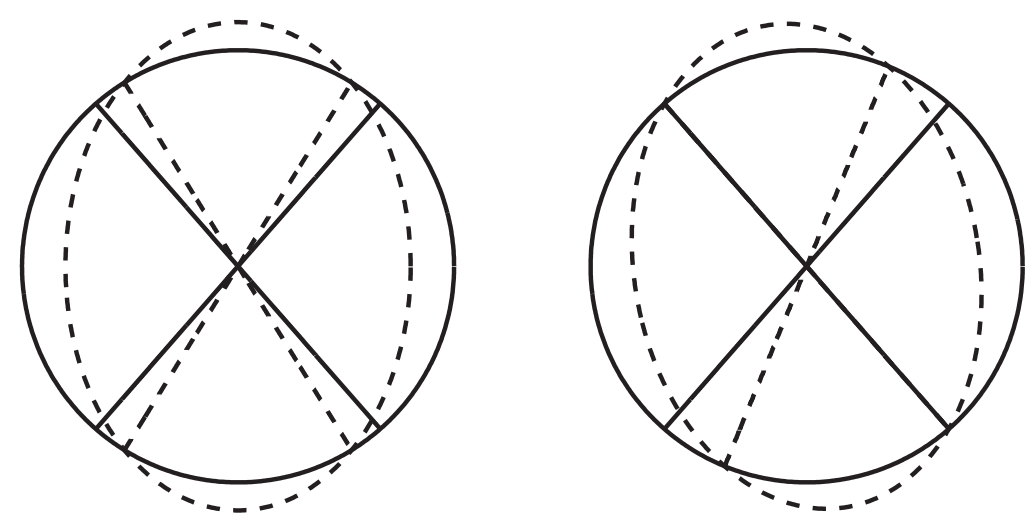

Figure 2.6: Illustration of the Bain distortion and the non-distorted lines (left). Bain distortion coupled with a rigid body rotation leaves an invariant line (right).

It is known that the deformation that arises during the transformation is an invariantplane strain (IPS) deformation, i.e. one that leaves an undistorted and unrotated plane. This suggests that the Bain distortion is not the only mechanism that is involved in the transformation process since it is an invariant-line strain. There are two possibilities for this second deformation, which has to be lattice invariant since the lattice correspondence was already achieved by the Bain distortion: twinning or slip. The total deformation tensor then becomes:

$$
\mathbf{F}=\mathbf{R} \cdot \mathbf{F}^{\prime}
$$


where

$$
\mathbf{F}^{\prime}=\mathbf{P}(g) \cdot \mathbf{B}
$$

in case of slip and:

$$
\mathbf{F}^{\prime}=\left[x \mathbf{B}_{1}+(1-x) \mathbf{R}_{\mathrm{T}} \cdot \mathbf{B}_{2}\right]
$$

in case of twinning. $\mathbf{P}$ is a simple shear deformation on the twinning plane by an amount $g, \mathbf{R}_{\mathrm{T}}$ is the rotation needed to ensure coherent twins and $x$ is the relative amount of twins.

The unknown parameters in Equations (2.4) and (2.5), $\mathbf{P}, \mathbf{R}_{\mathrm{T}}, g$ and $x$, can be determined by making use of geometrical analysis and continuum mechanics.

$\mathbf{R}_{\mathrm{T}}$ rotates a region that is deformed by $\mathbf{B}_{1}$ to have a coherent interface with the neighboring region, which is deformed by $\mathbf{B}_{2}$. This situation is sketched in Figure 2.7 , which shows two deformed regions in the projected $z=0$ plane $^{7}$. It is clear that the same deformation can be attained by two different mechanisms, twinning and slip, which also result in mathematically equivalent formulations.
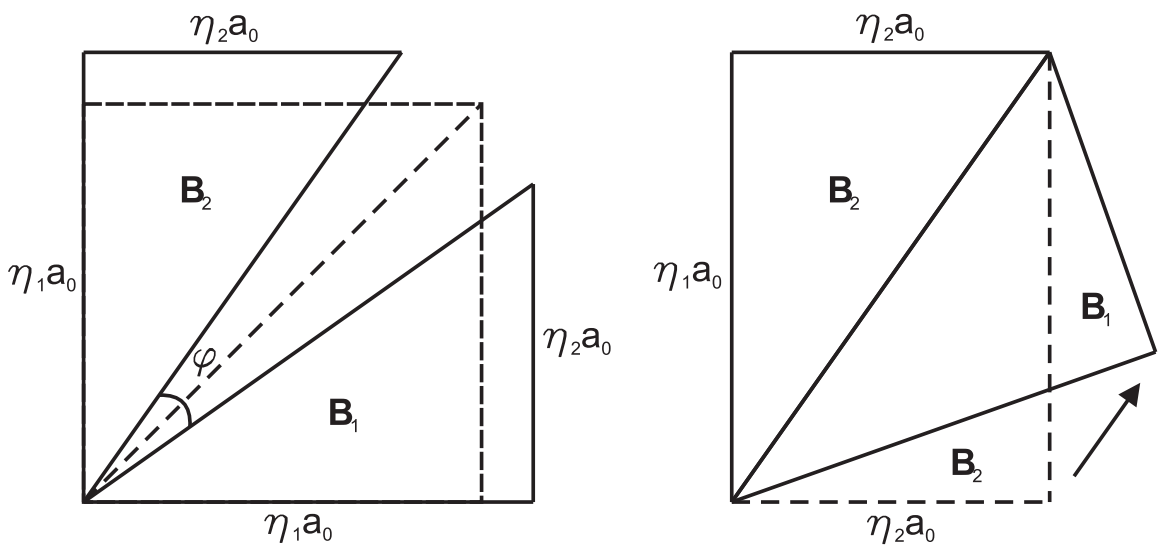

Figure 2.7: Twinning (left) and slip (right) as the lattice invariant strain that makes the total deformation an invariant-plane strain.

The IPS condition requires one of the principal stretches of deformation to be unity. Therefore, the corresponding principal strain and hence the determinant of the strain tensor will vanish:

$$
\begin{gathered}
\operatorname{det} \mathbf{E}=0, \\
\mathbf{E}=\frac{1}{2}\left(\mathbf{F}^{\mathrm{T}} \cdot \mathbf{F}-\mathbf{I}\right)
\end{gathered}
$$

where, $\mathbf{E}$ is the Green-Lagrange strain tensor.

$\mathbf{R}$ in Equations (2.4) and (2.5) does not affect the principal strains due to the fact that

$$
\mathbf{F}^{\mathrm{T}} \cdot \mathbf{F}=\mathbf{F}^{\prime \mathrm{T}} \cdot \mathbf{R}^{\mathrm{T}} \cdot \mathbf{R} \cdot \mathbf{F}^{\prime}
$$

\footnotetext{
${ }^{7}$ This is possible because the two regions have the same deformation on the perpendicular axis.
} 
and

$$
\mathbf{R}^{\mathrm{T}} \cdot \mathbf{R}=\mathbf{I}
$$

This makes it possible to solve Equation (2.6) for the unknowns $g$ or $x$ and calculate $\mathbf{F}^{\prime}$.

$\mathbf{F}^{\prime}$ is not symmetric, but making use of the polar decomposition theorem it can be written as the product of a rotation and a pure distortion:

$$
\mathbf{F}^{\prime}=\mathbf{Q} \cdot \mathbf{U}
$$

where $\mathbf{Q}$ is the rotation tensor.

Furthermore, the basis of $\mathbf{U}$ can be changed to the principal directions using the transformation tensor, $\Phi$ :

$$
\mathbf{U}=\boldsymbol{\Phi} \cdot \mathbf{U}_{\mathrm{d}} \cdot \boldsymbol{\Phi}^{\mathrm{T}}
$$

where $\mathbf{U}_{\mathrm{d}}$ represents $\mathbf{U}$ in the principal directions.

Any vector that lies on the invariant plane must preserve its magnitude after the deformation. Therefore:

$$
|\mathbf{F} \cdot \mathbf{r}|^{2}=|\mathbf{r}|^{2}
$$

which implies that:

$$
\left|\mathbf{U}_{\mathrm{d}} \cdot \mathbf{r}_{\mathrm{d}}\right|^{2}=\left|\mathbf{r}_{\mathrm{d}}\right|^{2}
$$

since rotation does not have any effect on the magnitude of a vector.

This can be written in component form as:

$$
\lambda_{1}^{2} x_{\mathrm{d}}^{2}+\lambda_{2}^{2} y_{\mathrm{d}}^{2}+\lambda_{3}^{2} z_{\mathrm{d}}^{2}=x_{\mathrm{d}}^{2}+y_{\mathrm{d}}^{2}+z_{\mathrm{d}}^{2}
$$

where $\lambda$ are the principal stretches. Since one of the principal stretches is unity, the following equation is obtained, which describes a plane in space:

$$
\begin{gathered}
\frac{z_{\mathrm{d}}}{y_{\mathrm{d}}}=-K, \\
K= \pm \sqrt{\frac{1-\lambda_{3}^{2}}{\lambda_{2}^{2}-1}}
\end{gathered}
$$

where $\lambda_{1}$ is assumed equal to unity. This plane has the normal $\mathbf{n}_{\mathrm{d}}$, with the following components in the principal directions:

$$
\left[\mathbf{n}_{\mathrm{d}}\right]=\frac{1}{\sqrt{1+K^{2}}}\left[\begin{array}{l}
0 \\
1 \\
K
\end{array}\right]
$$

In the basis of the austenite lattice it becomes

$$
\mathbf{n}=\boldsymbol{\Phi} \cdot \mathbf{n}_{\mathrm{d}}
$$


In the $x_{\mathrm{d}}$ direction there is no deformation since stretch in that direction is unity. This implies that the shear direction, $\mathbf{s}_{\mathrm{d}}^{\prime}$, must be perpendicular to $x_{\mathrm{d}}$. Since it is also perpendicular to $\mathbf{n}_{\mathrm{d}}$,

$$
\mathbf{s}_{\mathrm{d}}^{\prime}=\left[\begin{array}{l}
1 \\
0 \\
0
\end{array}\right] \times \mathbf{n}_{\mathrm{d}} .
$$

The magnitude of shear can be found by considering the extension of the normal during transformation to be a combination of shear and dilatation as,

$$
S^{2}=\left|\mathbf{U}_{\mathrm{d}} \cdot \mathbf{n}_{\mathrm{d}}\right|^{2}-\left(\operatorname{det} \mathbf{U}_{\mathrm{d}}\right)^{2}
$$

where, $S$ is the shear magnitude. Having established the magnitude and direction of the shear, the final deformation direction can be attained as follows:

$$
\begin{gathered}
\mathbf{s}_{\mathrm{d}}=S \mathbf{s}_{\mathrm{d}}^{\prime}+\left(\operatorname{det} \mathbf{F}_{\mathrm{d}}-1\right) \mathbf{n}_{\mathrm{d}}, \\
\mathbf{s}=\boldsymbol{\Phi} \cdot \mathbf{s}_{\mathrm{d}}
\end{gathered}
$$

The total deformation can now be established by

$$
\mathbf{F}=\mathbf{I}+\mathbf{s} \otimes \mathbf{n}
$$

The rotation tensor $\mathbf{R}$ can be found from:

$$
\mathbf{R}=\mathbf{F} \cdot \mathbf{F}^{\prime-1}
$$

which concludes determination of all the unknowns.

The above analysis yields 24 variants of the habit plane, shear direction, and transformation deformation. This is the number of the equivalent planes for a plane with non-zero and unequal indices. The multiplicity comes as a product of 3 Bain distortions, 2 solutions for $x$ or $g, 2$ possibilities for the twin plane and 2 solutions of Equation (2.14).

\subsection{Mechanically induced transformation}

It is known that the martensitic transformation can be affected by external mechanical factors, such as stress and strain. Previous research shows that the $M_{\mathrm{s}}$ temperature is shifted to higher temperatures when austenite is quenched under tensile stress. Additionally, it is observed that for steels with a metastable austenite phases present in the microstructure, martensitic transformation can be triggered by deformation at a constant temperature. There are several theories to explain these phenomena which will be summarized in the following sections. 


\subsubsection{Athermal transformation under stress}

The first theory to successfully describe the effect of stress on the $M_{\mathrm{s}}$ temperature was proposed by Patel and Cohen [70]. In that study the phenomenon was investigated experimentally and a theoretical basis to explain it was constructed. For a steel with the same composition, a linear relation between the magnitude of stress and the shift in $M_{\mathrm{s}}$ temperature was observed with the slope differing among different types of loading.

This linear relation can be rationalized considering several of the important features of martensitic transformation that were discussed in the previous sections. First of all, at a temperature higher than $M_{\mathrm{s}}$, thermodynamically there is not sufficient chemical driving force (free energy difference) to overcome the transformation energy barrier, $\left.\Delta G^{\gamma \rightarrow \alpha^{\prime}}\right|_{M_{\mathrm{s}}}$. This is schematically illustrated in Figure 2.8.

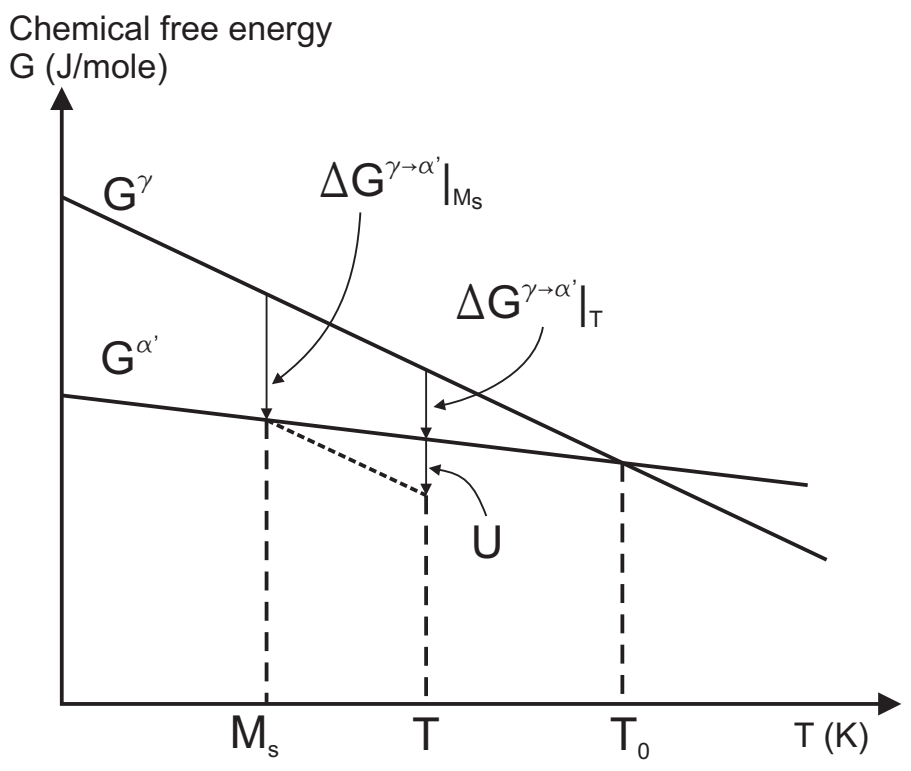

Figure 2.8: Schematic representation of the free energy difference at a temperature higher than $M_{\mathrm{s}}$ and the required additional amount of energy for transformation.

Thermodynamics suggests that the transformation can occur at higher temperatures than $M_{\mathrm{s}}$ if additional energy is supplied to the system. The additional energy can theoretically be of any kind provided that it aids the transformation, i.e. lowers the free energy of the system.

Because of the fact that transformation causes a deformation in the material, i.e. a combination of dilatation and shear, mechanical work is done provided that the transformation occurs under stress. Patel and Cohen interpreted this theory as

$$
U=\tau \gamma_{0}+\sigma \varepsilon_{0}
$$


where $\tau$ and $\sigma$ are the applied shear and normal stresses, $\gamma_{0}$ and $\epsilon_{0}$ are the transformation shear and dilatation strains, respectively. In tensorial notation,

$$
U=\sigma: \varepsilon^{\mathrm{tr}}
$$

where $\boldsymbol{\sigma}$ is the applied stress and $\varepsilon^{\text {tr }}$ is the transformation strain tensor.

They proposed that if

$$
\left.\Delta G^{\gamma \rightarrow \alpha \prime}\right|_{T}-U=\left.\Delta G^{\gamma \rightarrow \alpha \prime}\right|_{M_{\mathrm{s}}}
$$

holds then transformation would start.

For a given orientation of a martensitic variant and under uniaxial stress Equation (2.23) takes the following form:

$$
U=\frac{1}{2} \gamma_{0} \sigma_{1} \sin 2 \theta \cos \alpha \pm \frac{1}{2} \varepsilon_{0} \sigma_{1}(1+\cos 2 \theta)
$$

where $\theta$ is the angle between the uniaxial stress direction and the habit plane normal, $\mathbf{n}$, and $\alpha$ is the angle between the direction that would give the maximum shear on the plane, $\mathbf{s}_{\mathrm{m}}$ and the shear direction of the martensitic variant, $\mathbf{s}$, under consideration. The terms are explained schematically in Figure 2.9.

They calculated the maximum of $U$ for given $\gamma_{0}$ and $\epsilon_{0}$ values with respect to a uniaxial tensile stress state and showed that it is possible to calculate the change in $M_{\mathrm{s}}$ with respect to the applied stress quite accurately with this formula.

Olson and Cohen [67] generalized this behavior and based on the experimental results of Bolling and Richman [8], produced the schematic diagram shown in Figure 2.10. The diagram indicates the effect of applied tensile stress on the $M_{\mathrm{s}}$ including stress levels larger than the yield stress of austenite.

Two important points have to be mentioned. First, it is clear that stress raises the $M_{\mathrm{S}}$ temperature. Second, up to the yield point of austenite, the rise is linear with magnitude of stress. However, beyond the yield point the tendency deviates from linearity. The temperature at which this deviation occurs, i.e. the temperature at which the yield stress of austenite equals the stress that induces transformation, is denoted as $M_{\mathrm{s}}^{\sigma}$. It appears that in the plastic regime the stress required to start the transformation is less than would be predicted without plasticity. With further increase in the quench temperature, at a certain point it becomes impossible to initiate the transformation mechanically and this point is denoted as $M_{\mathrm{s}}^{\mathrm{d}}$.

There are several approaches to explain the phenomenon that beyond $M_{\mathrm{s}}^{\sigma}$ the stress required to induce the transformation drops below the stress-assisted transformation line. The first and the most widely accepted one is the Olson-Cohen theory [68] which states that in the plastic regime the number of available nucleation sites for martensitic transformation increases hence making it easier for martensite to form under stress. They propose that plastic strain increases the number of shear band intersections which act as martensitic embryos and hence, decrease the required energy, $U$, for transformation. The term strain-induced transformation is introduced in their study and this phenomenon is extended to describe deformation induced transformations as well. 


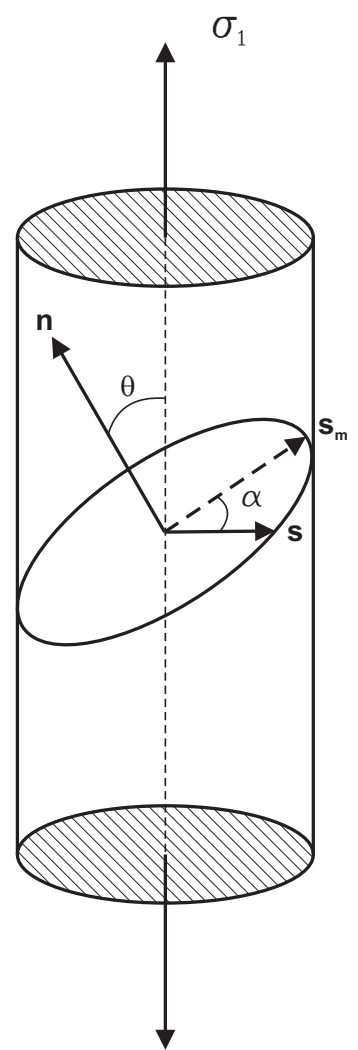

Figure 2.9: Mechanical driving force resolved on a martensitic variant with certain orientation with respect to the applied uniaxial stress.

The second approach remains loyal to the Patel-Cohen theory in that hereto stress is the main factor for supplying the mechanical energy for transformation. The addition is that when plastic deformation occurs in the material, due to the increase in the dislocation density, micro-stress fields occur due to the presence of dislocations which promote transformation locally.

Combining the two approaches one explanation for the occurrence of an $M_{\mathrm{s}}^{\mathrm{d}}$ temperature might be the combination of the softening of austenite and the increasing need for mechanical driving force with increasing temperature (see Figure 2.8). Consequently, $M_{\mathrm{s}}^{\mathrm{d}}$ would be the temperature at which the ultimate tensile stress of the austenite becomes less than the minimum stress required for inducing the transformation.

\subsubsection{Deformation-induced transformation}

It was demonstrated by Angel [1] that in some austenitic stainless steels it is possible to induce martensitic transformation isothermally (without the need for quenching) 


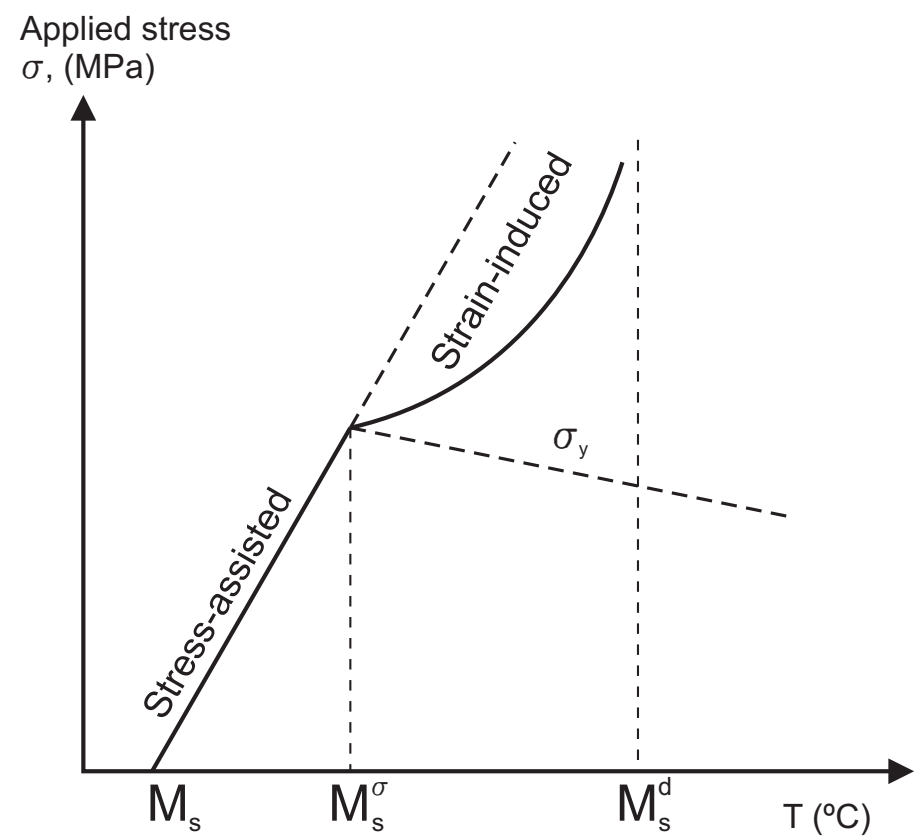

Figure 2.10: Schematic representation of the stress dependence of the $M_{\mathrm{s}}$ temperature in the stress-assisted and strain-induced regimes, after Olson and Cohen [67].

by the help of deformation. During a standard tensile test at temperatures around and below room temperature, he showed by resistivity measurements that the retained austenite in a stainless steel specimen transformed to martensite as shown in Figure 2.11 .

It is clear from the results that transformation is affected inversely by the ambient temperature, i.e. the higher the temperature the slower the kinetics. This is an expected result considering the thermodynamics of the phases as shown in Figure 2.3. Another important observation concerns the shape of the transformation curves: at all temperatures the curves are of sigmoid form, very similar to the isothermal transformation curves.

There are many phenomenological models $[74,81]$ that are based on observations that quite well simulate the deformation induced martensitic transformation. However, it must be mentioned that their contribution to the understanding of the underlying mechanisms of transformation is questionable.

One of the keystone studies that attempt to explain this phenomenon physically is by Olson and Cohen [68] and stems from the athermal transformation under stress tests that were described in the previous section. This theory suggests that during deformation in the plastic regime, additional nucleation sites are created for martensite. And the connection between plasticity and nucleation is provided by shear bands. Olson and Cohen proposed that when shear bands are created by 


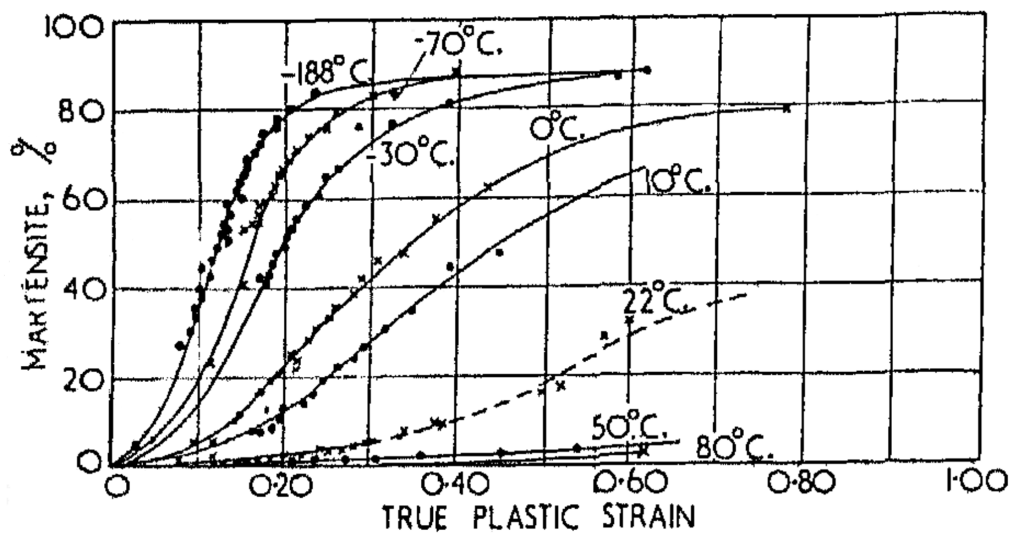

Figure 2.11: Amount of martensite formed by plastic strain at various temperatures, after Angel [1].

plastic deformation, at the intersection points of these bands martensitic embryos are created. Physically, these embryos can be stacking faults or epsilon martensite depending on the material and temperature under consideration. Since the embryos crystallographically form an intermediate step between austenite and martensite, they require less driving force to transform. There are recent micro-scale studies in which the effects of dislocation structures on martensitic embryo formation are modeled using for instance an Element-Free Galerkin method [51, 76]. Although the results theoretically promote the plastic strain induced transformation theory, the studies lack experimental verification.

The following are the equations for the evolution of the physical parameters and finally a phenomenological relation between the martensite volume fraction and accumulated plastic strain.

The evolution of the volume fraction of shear bands, $f^{\text {sb }}$, and hence the number of shear bands, $N_{\mathrm{v}}^{\mathrm{sb}}$, and their intersections, $N_{\mathrm{v}}^{\mathrm{I}}$, are given by the following equations:

$$
\begin{gathered}
\mathrm{d} f^{\mathrm{sb}}=\alpha\left(1-f^{\mathrm{sb}}\right) \mathrm{d} \varepsilon^{\mathrm{p}}, \\
N_{\mathrm{v}}^{\mathrm{sb}}=\frac{f^{\mathrm{sb}}}{\bar{v}^{\mathrm{sb}}}, \\
N_{\mathrm{v}}^{\mathrm{I}}=K\left(N_{\mathrm{v}}^{\mathrm{sb}}\right)^{n},
\end{gathered}
$$

where $K$ and $n$ are constants, $\bar{v}^{\text {sb }}$ is the average volume of a shear band, $\alpha$ is a temperature dependent parameter and $\varepsilon^{\mathrm{p}}$ is the equivalent plastic strain.

The evolution of the number of martensitic embryos is related to the evolution of the number of shear bands by a probability factor which physically represents whether or not the embryo has enough potency ${ }^{8}$ to transform. Once the number of embryos

\footnotetext{
${ }^{8}$ Minimum driving force for which a nucleation site will operate
} 
is known, the volume fraction of martensite can be calculated assuming a constant transformed volume of martensite for each embryo

$$
\begin{gathered}
\mathrm{d} N_{\mathrm{v}}^{\alpha^{\prime}}=p \mathrm{~d} N_{\mathrm{v}}^{\mathrm{I}}, \\
\mathrm{d} f^{\alpha^{\prime}}=\left(1-f^{\alpha^{\prime}}\right) \bar{v}^{\alpha^{\prime}} \mathrm{d} N_{\mathrm{v}}^{\alpha^{\prime}}
\end{gathered}
$$

where $p$ is the probability of an embryo to transform and it is given as a Gaussian distribution with respect to the available chemical driving force.

Finally, by substitution and integration of the above equations, the martensitic volume fraction as a function of plastic strain can be obtained as

$$
\begin{gathered}
f^{\alpha^{\prime}}=1-\exp \left(-\bar{v}^{\alpha^{\prime}} N_{\mathrm{v}}^{\alpha^{\prime}}\right), \\
\Rightarrow f^{\alpha^{\prime}}=1-\exp \left\{-\beta[1-\exp (-\alpha \varepsilon)]^{n}\right\}
\end{gathered}
$$

where

$$
\beta=\frac{\bar{v}^{\alpha^{\prime}} K}{\left(\bar{v}^{\mathrm{sb}}\right)^{n}} p .
$$

Equation (2.33) contains two temperature dependent parameters that are used for fitting, $\alpha$ and $\beta$. The model is fit to Angel's experiments and the temperature dependency of the parameters have been defined. The results of the model are given in Figure 2.12.

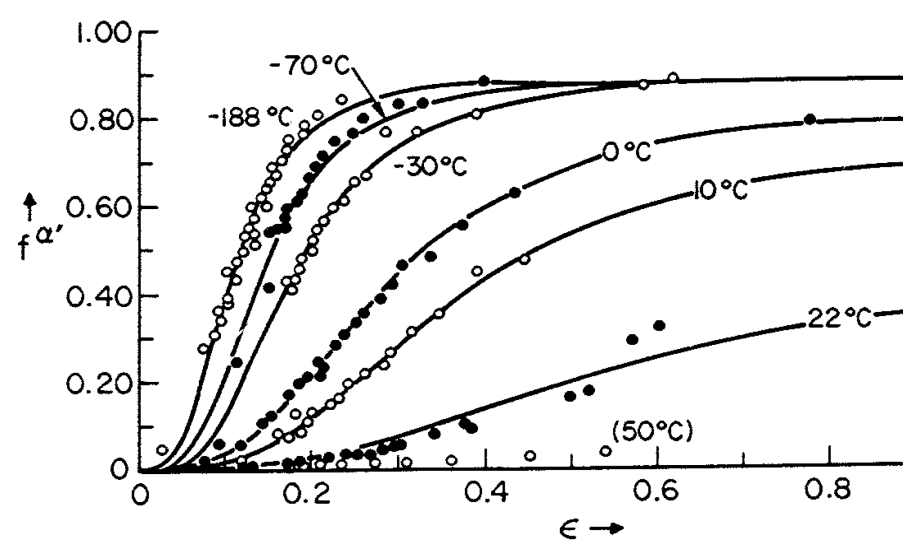

Figure 2.12: Results of the Olson-Cohen model with the best fit of the parameters $\alpha$ and $\beta$ to Angel's experiments [67].

Some improvements to the Olson-Cohen model were made by Stringfellow, Parks and Olson [80] to include the stress-state effects and transformation plasticity.

Since for a constant equivalent plastic strain the temperature and stress state may change, which in turn changes the probability of an embryo to transform, the following modification is included in the evolution equation of number of embryos:

$$
\mathrm{d} N_{\mathrm{v}}^{\alpha^{\prime}}=p \mathrm{~d} N_{\mathrm{v}}^{\mathrm{I}}+N_{\mathrm{v}}^{\mathrm{I}} \mathrm{d} p \mathrm{H}(\mathrm{d} p) .
$$


The Heaviside function, $\mathrm{H}(\mathrm{d} p)$, had to be included in Equation (2.35) to convert the physical irreversibility into a mathematical expression. This means that transformation will take place only if the probability increases.

The probability distribution is given by the following Gaussian distribution function, which is a function of stress state and temperature through the $g$ parameter which resembles the definition of a total driving force:

$$
p=\frac{1}{s_{g} \sqrt{2 \pi}} \int_{-\infty}^{g} \exp \left[-\frac{1}{2}\left(\frac{g^{\prime}-\bar{g}}{s_{g}}\right)^{2}\right] \mathrm{d} g^{\prime}
$$

with,

$$
\begin{gathered}
g=g_{0}-g_{1} \Theta+g_{2} \Sigma, \\
\Theta=\frac{T-M_{\mathrm{s}}^{\sigma}}{M_{\mathrm{s}}^{\mathrm{d}}-M_{\mathrm{s}}^{\sigma}}, \\
\Sigma=\frac{-\sigma^{\mathrm{h}}}{\sqrt{3} \bar{\tau}},
\end{gathered}
$$

where $s_{g}$ and $\bar{g}$ are constants for the standard deviation and the mean value of the distribution respectively, and $g_{0}, g_{1}$ and $g_{2}$ are constants. Equation (2.36) gives the probability of an embryo to transform at a certain temperature, $T$, and triaxiality, $\Sigma$, through integration of the distribution function from $-\infty$ to $g$ where, $g$ is the temperature and stress state dependent term. The probability distribution therefore is constant but the upper bound for the integration is a parameter.

This theory has further been modified and improved to reflect the effects of stressstate, strain rate and temperature more physically and accurately $[40,86]$. The strain-rate and stress-state sensitivity is added to the evolution of shear bands and the latent heat of transformation is added to the thermal part of the calculations. Olson-Cohen theory was implemented and applied by many authors for large scale finite element analysis most of which resulted in satisfactory simulations[77, 87].

Another theory for deformation-induced martensitic transformations was proposed by Tamura [82]. In his model, stress is still considered as the main reason for the transformation, in line with the Patel-Cohen theory. He considered the Patel-Cohen equation for a distribution of grain orientations in a polycrystal and integrated over the angle that the grains make with the applied stress. He finally obtained the following equation:

$$
f^{\alpha^{\prime}}(\sigma)=\frac{1}{\alpha_{0}} \int_{0}^{\alpha_{0}} \frac{2}{\pi} \int_{0}^{\pi / 2} C\left\{\frac{\sigma}{2}\left[\gamma_{0} \sin 2 \theta \cos \alpha \pm \varepsilon_{0}(1+\cos 2 \theta)\right]-U^{\prime}\right\} \mathrm{d} \theta \mathrm{d} \alpha
$$

where $f^{\alpha^{\prime}}$ is the martensite volume fraction, $C$ is a constant, $U^{\prime}$ is the critical energy barrier at this temperature, i.e. $\left.\Delta G^{\gamma \rightarrow \alpha^{\prime}}\right|_{T}-\left.\Delta G^{\gamma \rightarrow \alpha^{\prime}}\right|_{M_{\mathrm{s}}}$.

However, in Equation (2.40) there is a problem because when the integrations are performed, the attained value has energy units, and does not converge to 1 with an infinite stress magnitude. 
The correct equation should take the integral over the angles where the net driving force is positive but not multiplied by the driving force. The correction can be made by introducing a Heaviside function as follows:

$$
f^{\alpha^{\prime}}(\sigma)=\frac{1}{\pi} \int_{0}^{\pi} \frac{2}{\pi} \int_{0}^{\pi / 2} C \mathrm{H}\left\{\frac{\sigma}{2}\left[\gamma_{0} \sin 2 \theta \cos \alpha \pm \varepsilon_{0}(1+\cos 2 \theta)\right]-U^{\prime}\right\} \mathrm{d} \theta \mathrm{d} \alpha
$$

where $\mathrm{H}$ is the Heaviside function.

It can be deduced from Equation (2.41) that according to Tamura, the gradual increase of the martensite fraction is associated with the increase of applied stress through strain hardening as opposed to the creation of nucleation sites as proposed by Olson and Cohen.

Although in Equation (2.41) only a uniaxial stress state is considered for simplicity, the theory can also be generalized to a multiaxial stress state. When there is stress acting on the material, according also to Patel and Cohen there is a resolved mechanical driving force that would aid the transformation. Patel and Cohen were interested in the maximum of this driving force since only the calculation of the $M_{\mathrm{S}}$ temperature was considered. However, for the evolution of martensite, not only the maximum but the complete distribution of this driving force throughout the material is important. Tamura considered a polycrystal with infinitely many grains with random orientations homogeneous in every direction. Therefore, under the uniaxial stress, transformation will be promoted on habit planes with an angle of up to $\pi / 2$ and shear directions up to a certain $\alpha_{0}$ (see Figure 2.9). Outside this range however, transformation will not take place. Hence, the weighted integral of the driving force over these angles minus the energy barrier gives the martensitic volume fraction. In the corrected Equation (2.41) there is no longer need for the calculation of $\alpha_{0}$ since the Heaviside function eliminates the unfavorable orientations naturally.

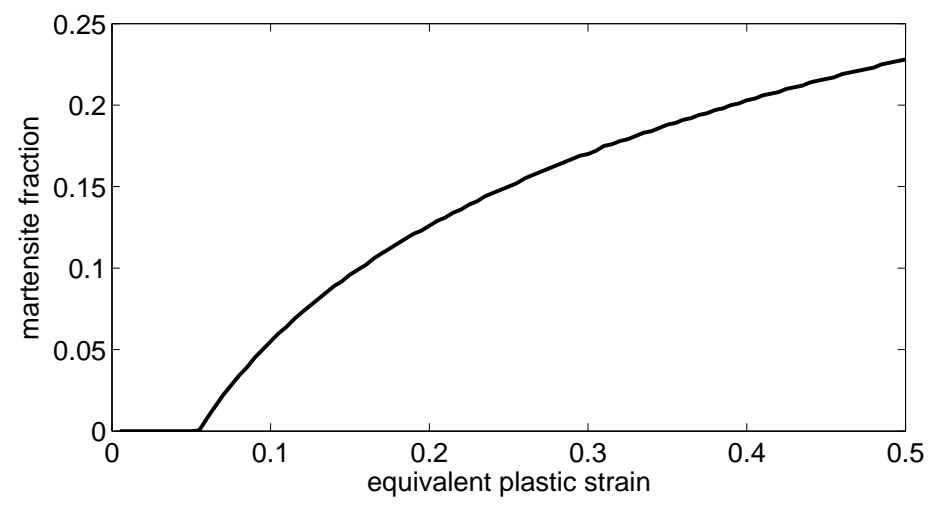

Figure 2.13: Result of the model proposed by Tamura, with an assumed power law hardening material and an arbitrary energy barrier.

Equation (2.41) is plotted in Figure 2.13 with the stress assumed to follow a power law relation with the plastic strain. The plot shows that the predicted 
transformation is much slower compared to experimental observations. The reason of the underestimation lies in the departure point of the model, where the orientation of only one variant throughout the material is considered. It is known that in an austenite crystal there are multiple martensitic variants that can transform. This makes a significant difference in the evolution of the martensite fraction with stress, since these variants in the crystal are not directionally independent.

According to Tamura, the strain-induced regime as defined by Olson-Cohen during an athermal transformation under stress is not caused by the generation of nucleation sites but by stress concentration around crystallographic obstacles.

\subsection{TRansformation Induced Plasticity (TRIP)}

It was observed experimentally that when a phase transformation occurs under small stress, in some alloys a net unrecoverable strain occurs along the direction of the applied stress, although the stress itself is not high enough to cause any plastic deformation [26]. This phenomenon was observed for different types of phase transformations, not necessarily martensitic.

The first theory to explain this behavior was proposed by Greenwood and Johnson. Their theory is based on consideration of the micro-stresses that develop during a volumetric expansion of a certain region in the parent phase. They consider the expansion to happen concurrently with an application of a small stress. In absence of an applied stress, the stresses that arise because of the mismatch of the volumes cancel out, without producing a net shape change apart from dilatation. However, when an external stress field is imposed on the micro-stress fields plastic deformation can be induced locally. Their formulation results in the calculation of the net TRIP strain as

$$
\varepsilon^{\mathrm{tp}} \approx \frac{5}{6} \frac{\delta v}{v} \frac{\sigma}{\sigma^{\mathrm{y}}}
$$

where $\delta v / v$ is the volumetric strain and $\sigma^{\mathrm{y}}$ is the yield stress of the soft parent phase.

This equation can be generalized to three dimensional stress states in a straightforward manner:

$$
\varepsilon^{\mathrm{tp}} \approx \frac{5}{6} \frac{\delta v}{v} \frac{\boldsymbol{\sigma}^{\prime}}{\sigma^{\mathrm{y}}}
$$

where $\boldsymbol{\sigma}^{\prime}$ is the deviatoric part of the stress tensor.

They validated their equation for the transformation of Uranium, Zirconium and Titanium sheets and rods. However, these transformations were not martensitic in nature.

Magee performed tests on Fe-Ni alloys to quantify the transformation plasticity that is observed during the martensitic phase transformation $[57,58]$. He did relaxation tests under tension during the transformation and by eliminating all other effects measured the net inelastic strain caused by the transformation. He showed that the Greenwood-Johnson model failed to describe the large strains that are attained in the 
experiments. The discrepancy between the Greenwood-Johnson prediction and the experiments were around an order of magnitude.

Therefore, he proposed another mechanism to describe the underlying physics of the phenomenon. His derivations start with considering the amount of transformation strain that would be caused by an individual martensitic variant, following the PatelCohen theory,

$$
\varepsilon^{\operatorname{tr}}(\theta)=\frac{1}{2} \gamma_{0} \sin 2 \theta+\frac{1}{2} \varepsilon_{0}(1+\cos 2 \theta)
$$

where, $\varepsilon^{\operatorname{tr}}(\theta)$ is the strain associated with the martensitic variant of certain orientation, $\theta$, with respect to the applied stress.

To find the amount of strain per unit of stress-assisted transformation, the following averaging is performed:

$$
\bar{\varepsilon}^{\operatorname{tr}}=\frac{\int_{\theta} \varepsilon^{\operatorname{tr}}(\theta) f^{\sigma}(\theta) \mathrm{d} \theta}{\int_{\theta} f^{\sigma}(\theta) \mathrm{d} \theta}
$$

where $f^{\sigma}(\theta)$ defines the orientation dependent volume fraction of martensite formed. However, the orientation dependency comes from the favorability of the variant hence, $f^{\sigma}(\theta)$ can be decomposed as follows:

$$
\begin{gathered}
f^{\sigma}(\theta)=f^{\prime} N(\theta) \\
N(\theta)=\rho U(\theta) \\
U(\theta)=\sigma \varepsilon^{\operatorname{tr}}(\theta)
\end{gathered}
$$

where $f^{\prime}$ is the transformed volume fraction per plate, $N(\theta)$ is the number of plates that form, $\rho$ is a proportionality constant between the amount of driving force in a certain orientation and the number of plates that form in that orientation and $U$ is the driving force as defined by Patel and Cohen.

Combining Equations (2.45) to (2.48) and considering that transformation only occurs between certain orientations, the following definition for the transformation strain is obtained:

$$
\bar{\varepsilon}^{\operatorname{tr}}=\frac{\int_{\theta_{0}}^{\frac{\pi}{2}}\left(\varepsilon^{\operatorname{tr}}(\theta)\right)^{2} \sin \theta \mathrm{d} \theta}{\int_{\theta_{0}}^{\frac{\pi}{2}} \varepsilon^{\operatorname{tr}}(\theta) \sin \theta \mathrm{d} \theta}
$$

The results of Equation (2.49) using the Patel-Cohen parameters for $\gamma_{0}$ and $\varepsilon_{0}(0.2$ and 0.04 , respectively) yields a transformation strain of the order of 0.09 , or $9 \%$, which closely matches the experimentally observed values. The TRIP strain calculation by Magee has shown that there is definitely a selection mechanism of martensitic variants during transformation under stress.

\subsection{Summary}

In this chapter the literature and the currently accepted theories on martensitic transformation and mechanical effects have been briefly discussed. 
Martensitic transformation is a fast, diffusionless transformation which preserves the chemical composition but alters the shape of the lattice with an invariant-plane strain deformation. This deformation takes place on habit planes and in certain preferred directions. Therefore, if this deformation occurs under stress, mechanical work is generated which in turn reduces the free energy of the system. Selective transformation of these variants results in a net deviatoric strain in the direction of the applied stress. Additionally, the volumetric expansion of martensite plates can generate microstresses that cause localized plastic deformation with a preferential direction.

Plastic strain is thought to create potential martensite nucleation sites, or embryos, on which transformation is easier. Evolution of the number of embryos can be related to the evolution of the number of shear band intersections via a phenomenological model leading to the strain-induced transformation model.

It can be concluded that in the literature there are two competing theories. One focuses on the effect of strain in creating martensitic embryos for aiding the transformation and hence this will be referred to as strain-induced transformation theory. The other theory focuses on the importance of the mechanical driving force; hence, it is claimed that stress is the main cause for transformation. 



\section{3}

\section{Experiments}

In the previous chapter it was shown that in the literature there are two competing theories to describe the mechanically induced martensitic transformation phenomenon in metastable austenitic stainless steels. One theory suggests that the transformation is due to applied stress, which creates additional driving force to overcome the energy barrier for transformation. The alternative theory proposes that since martensite nucleates heterogeneously, the mechanical effect is due to the creation of new embryos with the accumulation of plastic strain.

Therefore, in this chapter some mechanical tests are presented that shed more light on the effects of plastic strain and stress on the transformation kinetics of the steel under consideration. Two types of mechanical tests were carried out: biaxial deformation tests and prestrain tests.

\subsection{Material}

The material used in this study is $12 \mathrm{Cr}-9 \mathrm{Ni}-4 \mathrm{Mo}$ (ASTM A 564) austenitic stainless steel with the nominal composition given in Table 3.1. The retained austenite can be almost completely transformed into martensite by deformation at room temperature. Furthermore, the transformed martensite can be hardened by aging (precipitation hardening) which makes this material a maraging steel as well.

The material was in sheet metal form and fully austenitic in as-received condition. For the biaxial tests, due to the complexity of the geometry, test samples were obtained by spark erosion; for the uniaxial tests, a standard cutting operation was performed. Samples were kept at a constant $80^{\circ} \mathrm{C}$ until the tests; hence no prior isothermal transformation was observed in the samples. The transformation characteristics of this steel were studied thoroughly in [74] by uniaxial tests at different temperatures. 
Table 3.1: Chemical composition of the steel (ASTM A 564) used in the experiments in $\mathrm{wt} \%$.

\begin{tabular}{lr} 
Element & wt. \% \\
\hline $\mathrm{C}+\mathrm{N}$ & $<0.05$ \\
$\mathrm{Cr}$ & 12.0 \\
$\mathrm{Ni}$ & 9.1 \\
$\mathrm{Mo}$ & 4.0 \\
$\mathrm{Cu}$ & 2.0 \\
$\mathrm{Ti}$ & 0.9 \\
$\mathrm{Al}$ & 0.4 \\
$\mathrm{Si}$ & $<0.5$ \\
\hline
\end{tabular}

\subsection{Magnetic sensor}

The transformation from austenite to martensite is monitored with a magnetic sensor utilizing the permeability difference of the two phases which is of the order of 100 [65, 74]. The sensor supplies a steady and representative signal that measures the amount of the martensite phase throughout the experiment. This signal can be disturbed by several factors which are removed by a calibration procedure. It is stated in [74] that temperature as well as the stress and strain affect the permeability of martensite due to the magnetostriction phenomenon. In addition, the tool steel clamps used in these tests influence the signal. Therefore, the recorded signal is post-processed to eliminate these disturbances. Once a clean signal is obtained, a correlation with the actual amount of martensite is performed by metallographic inspection. Important points in the calibration process have been summarized in Appendix $\mathrm{C}$ and more details can be found in [71].

An important step before inspection is the freezing of the microstructure by an aging treatment. It has been observed that austenite continues to transform isothermally after the tests if no precaution is taken. Hence, all samples were heated and kept at $500{ }^{\circ} \mathrm{C}$ for 30 minutes immediately after the tests. It is known that this is the aging temperature of martensite (precipitation hardening); however it is not clear why this treatment stabilizes the austenite phase.

The samples were cut through longitudinally and polished using standard metallographic techniques after which the color etchant Lichtenegger-Blöch solution was used which provides an adequate amount of contrast between the austenite and martensite phases for quantification by a standard image processing tool. One of the images used for correlation is presented in Figure 3.1.

\subsection{Effect of stress on transformation kinetics}

It is common knowledge that stress has an effect on the transformation kinetics. There have been studies to model this behavior but the number of experimental studies that can specifically demonstrate the effect is small. 


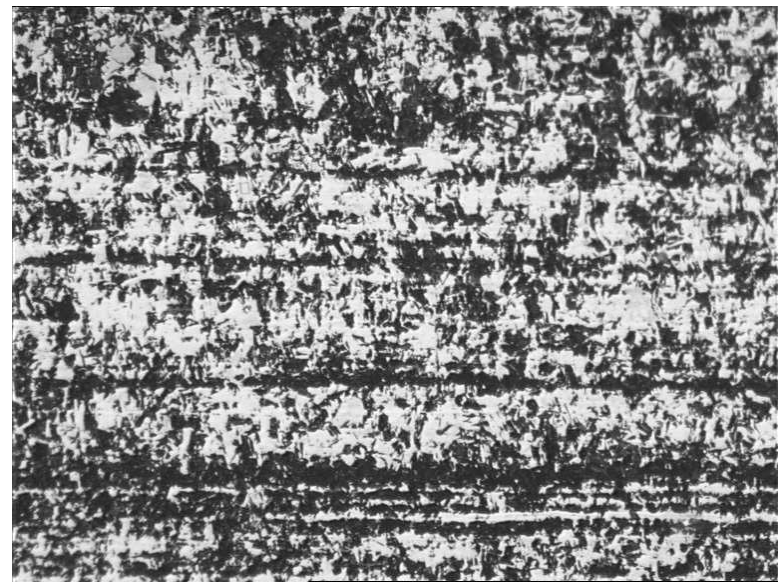

Figure 3.1: A metallographic image showing 59\% martensite (dark).

In the literature, mostly tests in which different points in the material undergo different deformation paths are considered, e.g. single-shear tests [28]. In these types of experiments due to the nature of the test setup, different stress states are applied in different points on the material and unfortunately time-dependent transformation monitoring lacks in most of them [41, 50, 91]. There are also athermal transformation tests that have been carried out under multiaxial loading (a combination of tension and torsion) of the specimen which gives an idea of the effect of the stress state on the kinetics [15, 88].

The aim of this part of the study is therefore to quantify the effect of the stress state on transformation kinetics. It is obvious that in order to achieve this, a real-time monitoring of the martensite amount during the tests is necessary. Furthermore, a distinct definition of the stress-state must be provided and the experiments must be able to reflect this definition. Finally, the results of different tests must be comparable to quantify the effect.

The expectation from the results is to understand the relation between stress and transformation. It is known from Patel-Cohen theory that stress provides the driving force for transformation. In the strain-induced theory however, there is no direct relation of the transformation rate and stress. On the other hand, there have been studies to incorporate this effect by relating stress triaxiality and shear band formation kinetics $[80,86]$. Therefore, the aim is to test these theories and if possible contribute to the validation of them.

\subsubsection{Biaxial tests}

The biaxial test facility is schematically represented in Figure 3.2. Two separate clamps constrain the upper and the lower sections of the sample. The upper clamp can move only in the horizontal direction whereas the lower clamp can move only 


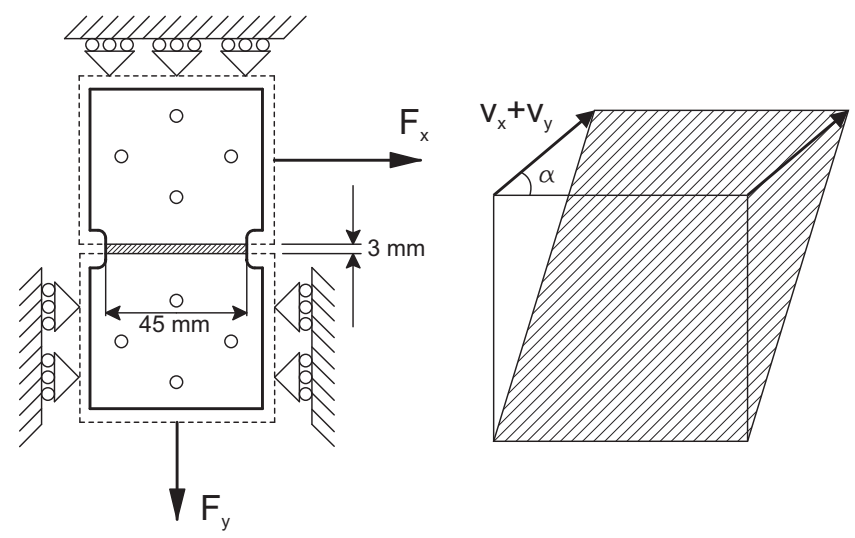

Figure 3.2: Schematic representation of the test facility (left) and the deformation zone (right).

vertically. The horizontal and vertical displacements are controlled independently via separate actuators.

With this setup it is possible to impose a constant stress state on the deformation zone during the test by keeping the direction of the deformation rate constant. This direction is controlled via the relative speed of the clamps as illustrated in Figure 3.2. It is possible to impose a range of stress states on the material starting from plane strain tension to simple shear as shown in Figure 3.3.
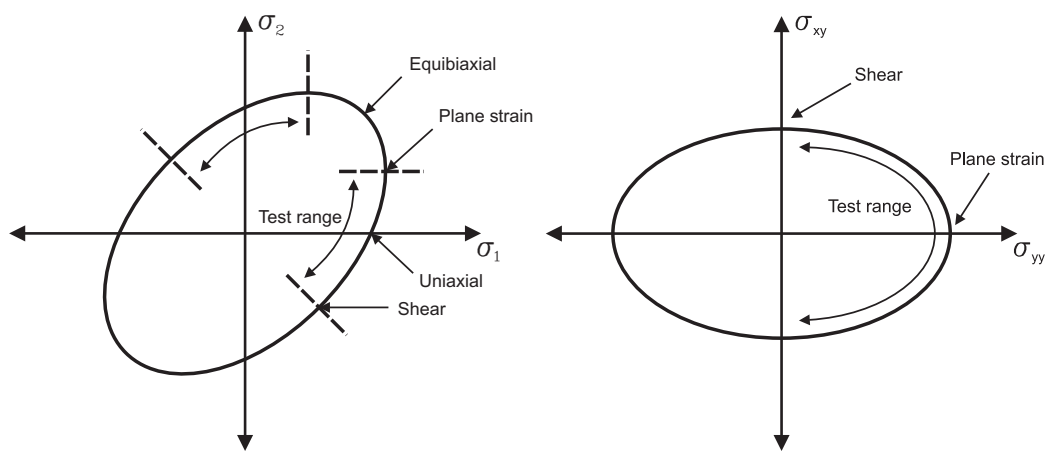

Figure 3.3: Schematic illustrations of the range of stress states that can be imposed on the sample.

\subsubsection{Stress state and strain}

The dimensions of the deformation zone $(\mathrm{w}=45 \mathrm{~mm}, \mathrm{~h}=3 \mathrm{~mm}, \mathrm{t}=0.5 \mathrm{~mm})$ project the stresses onto the two-dimensional principal stress space between the plane strain tension and simple shear points. The horizontal load cell of the setup provides the 
data for the shear component of stress, $\tau_{x y}$, and the vertical load cell provides the tensile data, $\sigma_{y y}$. The horizontal stress component, $\sigma_{x x}$, cannot be measured on this facility. However, it is assumed based on basic theories on the mechanics of materials that the horizontal stress is always proportional to the vertical stress: $\sigma_{x x}=\nu \sigma_{y y}$

The strain was measured in real-time on the material surface using a camera and dot-tracking software. 16 black dots were applied to the specimen surface before the test and the corresponding positions were recorded at a frequency of approximately 10 frames/sec. The data were post-processed and averaged to find the strain that accumulated in the material. The approximate resolutions of the strain and stress measurements were $0.05 \%$ and $2 \mathrm{MPa}$, respectively.

With this setup it is not possible to measure the strain in the thickness direction. The equivalent strain calculations presented in the figures are based on a volume conservation assumption, although it is known that volume is not conserved during the martensitic transformation; the volume change is of the order of $2 \%$.

\subsubsection{Tests}

Two different types of tests were conducted with the Biaxial tester: proportional and non-proportional. During the first type of tests, as the name suggests, the ratio of the vertical and horizontal clamp speeds was kept constant. This situation makes it possible to have a constant stress state in the deformation zone throughout the complete deformation route. By changing the proportionality constant, different stress states as shown in Figure 3.3 can be imposed. In order to have a solid parameter defining the different tests, the arc tangent of the deformation direction is used which will be referred to as the deformation angle, $\alpha$ :

$$
\alpha=\arctan \left(\frac{v_{y}}{v_{x}}\right)=\arctan \left(\frac{D_{y y}}{2 D_{x y}}\right)
$$

where $v_{x}$ and $v_{y}$ are the clamp speeds as illustrated in Figure $3.2, D_{y y}$ and $D_{x y}$ are the tensile and shear deformation rates.

In the second type of tests, after a certain amount of shear deformation, the direction of deformation was changed suddenly. In other words, in the initial stage of deformation the deformation angle was set to $\alpha_{1}=0$ and in the second stage it was kept in the range $0<\alpha_{2}<150$.

\subsubsection{Proportional deformation}

The stress space that falls between simple shear and plane strain was covered in 8 different stress states. The deformation angle was adjusted to cover the space evenly. The results of the tests are presented in the Figures 3.4, 3.5 and 3.6 for $\sigma_{y y}-\varepsilon^{\mathrm{eq}}$, $\tau_{x y}-\varepsilon^{\mathrm{eq}}$ and $f^{\alpha^{\prime}}-\varepsilon^{\mathrm{eq}}$, respectively.

Assuming that the material follows an isotropic yield behavior in the plastic regime, it is possible to estimate the unknown $\sigma_{x x}$ component of the stress tensor as $\sigma_{x x}=\frac{1}{2} \sigma_{y y}$. 


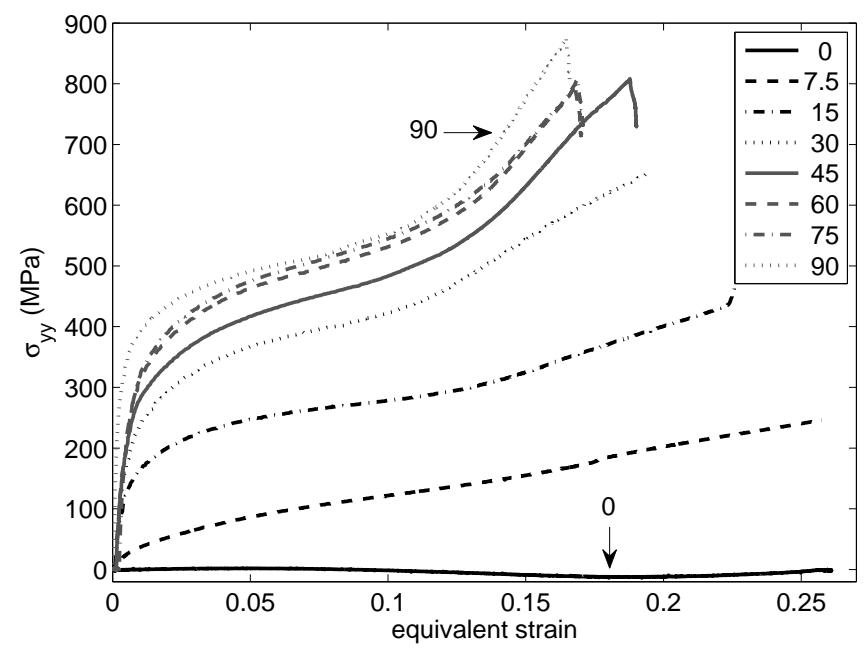

Figure 3.4: Tensile stress vs. equivalent strain plots for varying $\alpha$.

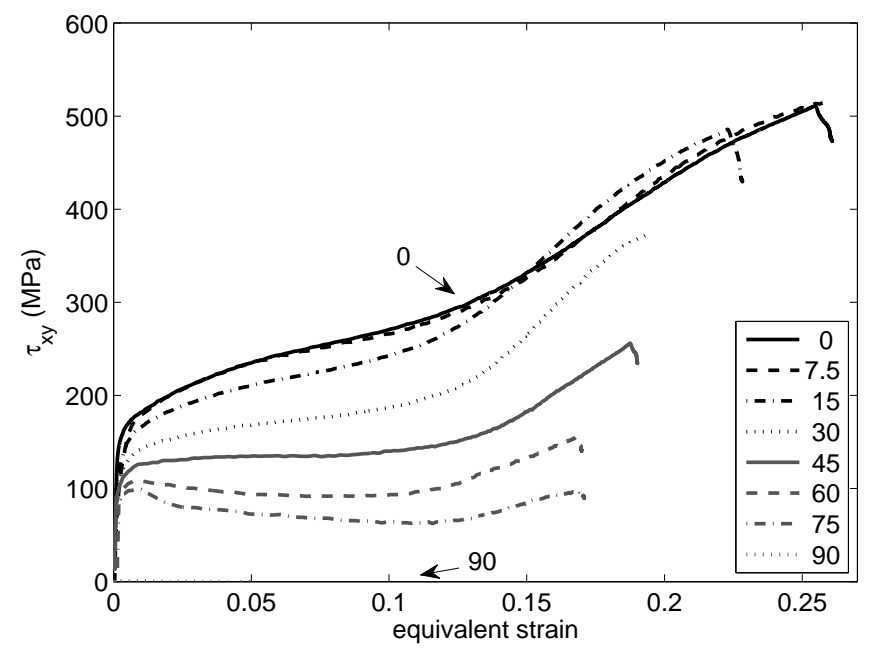

Figure 3.5: Shear stress vs. equivalent strain plots for varying $\alpha$.

Since the other components of the stress can safely be assumed to be 0 , the equivalent Von Mises stress can be computed. The resulting computed equivalent stress vs. equivalent strain curves are shown in Figure 3.7.

Figure 3.6 shows that there is an influence of the stress state on the transformation behavior. It is observed that the rate of transformation increases with the amount of tension although the shape of the curves is similar. This implies that to induce the 


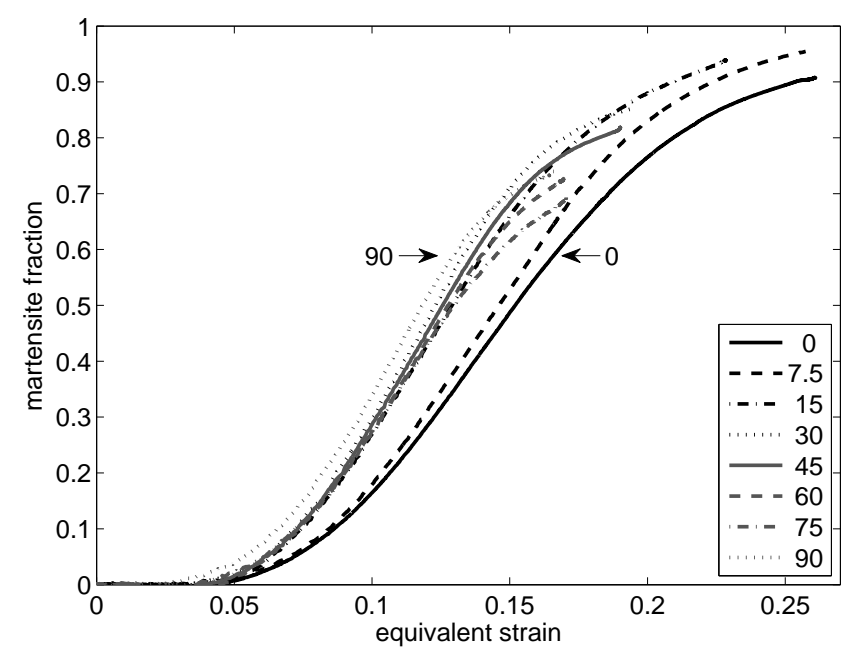

Figure 3.6: Martensite fraction vs. equivalent strain plots for varying $\alpha$.

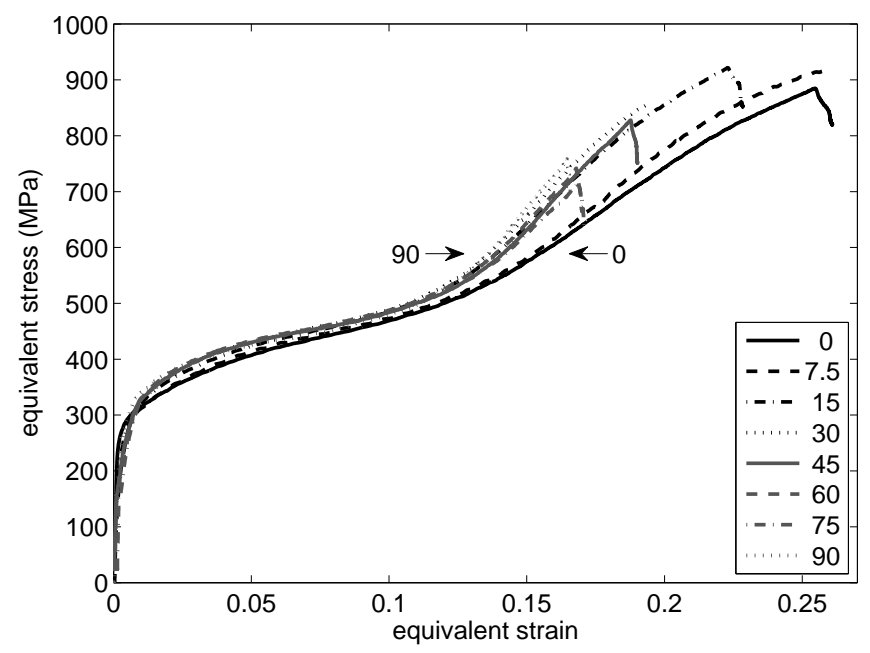

Figure 3.7: Equivalent stress (based on Von Mises assumption) vs. equivalent strain plots for varying $\alpha$.

same amount of martensite, more strain is needed in shear than in tension. This is an expected result since the Patel-Cohen theory predicts more driving force in tension than in shear.

In Figure 3.7, the characteristic stress-strain behavior of this steel can be observed. It is known that at higher temperatures, i.e. in the absence of transformation, the 
material follows a monotonic hardening behavior. However, when transformation does take place, the hardening behavior is significantly altered as revealed by the experiments.

Beyond the yield stress, a plateau is observed where the material seems to show no hardening. This plateau is the natural outcome of the transformation plasticity phenomenon. When transformation takes place, the additional inelastic (stress-free) strain that comes from the transformation, i.e. TRIP, causes less plastic strain to resolve hence the material shows less strain hardening. With more deformation, a significant increase in the hardening rate is observed which is due to the fact that the alloy becomes more martensitic than austenitic and consequently the mechanical behavior changes from that of austenite to that of martensite.

\subsubsection{Non-proportional deformation}

The aim of the non-proportional tests was to see the effect of a sudden strain path change on the transformation kinetics. To observe this, the samples were first deformed in shear up to a level that induces approximately $50 \%$ martensite. After this the deformation path was changed to an $\alpha_{2}$ between $0^{\circ}$ and $150^{\circ}$. This range was covered in 6 steps with $30^{\circ}$ intervals. The results are shown in Figures 3.8, 3.9 and 3.10 for $\sigma_{y y}-\varepsilon^{\mathrm{eq}}, \tau_{x y}-\varepsilon^{\mathrm{eq}}$ and $f^{\alpha^{\prime}}-\varepsilon^{\mathrm{eq}}$, respectively.

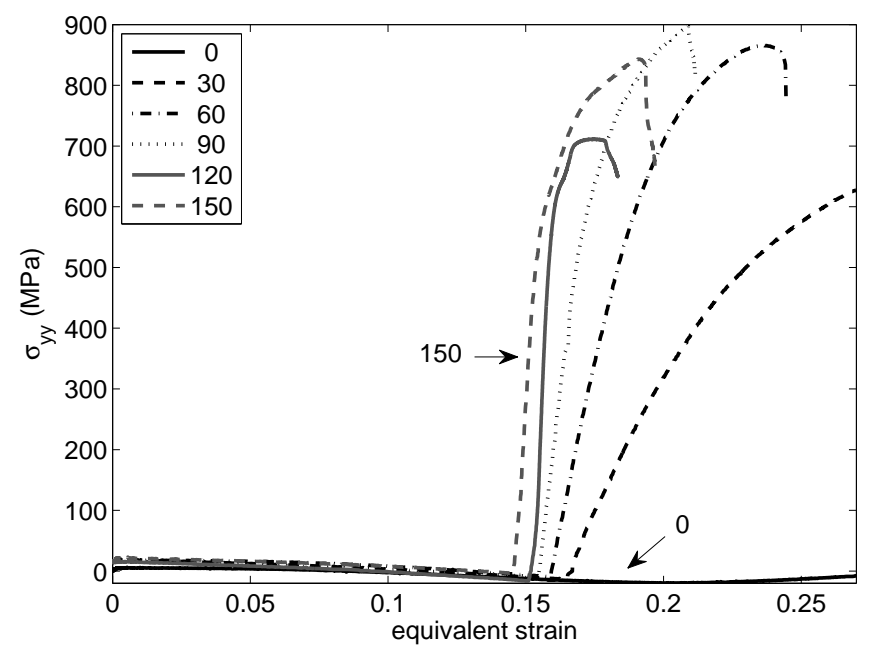

Figure 3.8: Tensile stress vs. equivalent strain plots for varying $\alpha_{2}$ after an initial shear deformation.

As a start, in Figure 3.10 it is clear that the first stage of the deformation process has been reproduced very well in all the tests. All samples resulted in the same amount of martensite at the end of the first stage. 


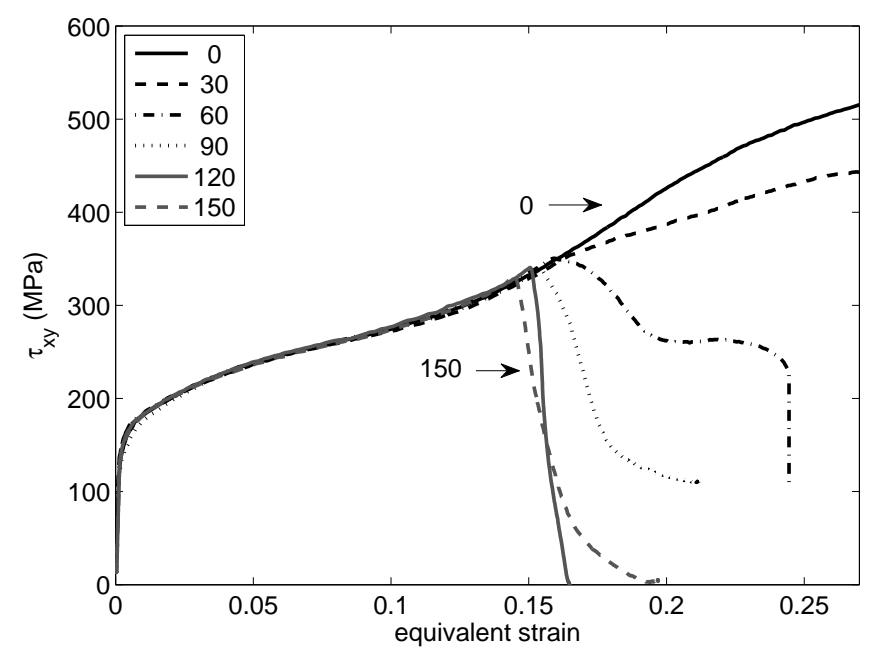

Figure 3.9: Shear stress vs. equivalent strain plots for varying $\alpha_{2}$ after an initial shear deformation.

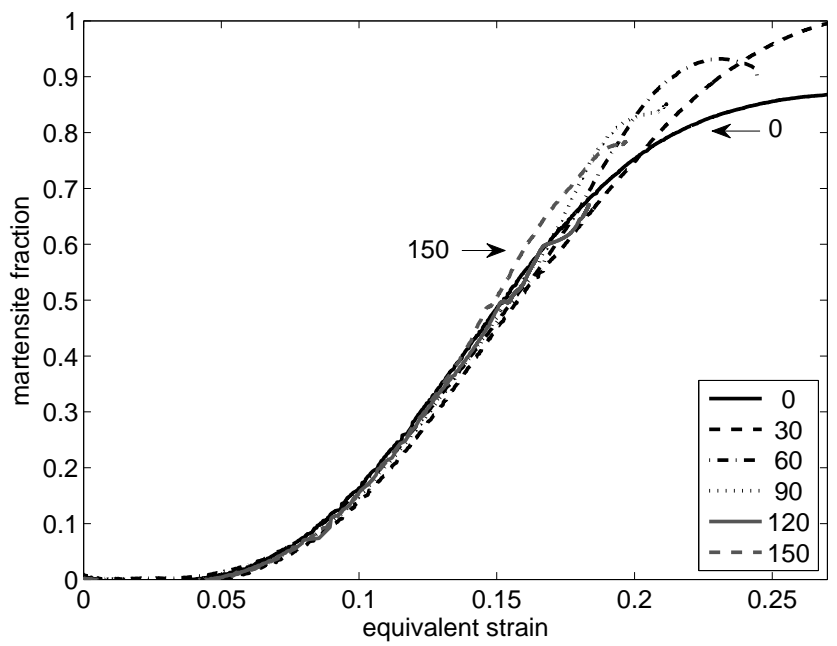

Figure 3.10: Martensite fraction vs. equivalent strain plots for varying $\alpha_{2}$ after an initial shear deformation.

The aim of the strain path change was to observe the transformation behavior when the stress state is changed suddenly. Patel-Cohen theory suggests that the driving force is related to the orientation of the martensitic variants with respect to the applied stress direction. Therefore, if the stress direction is suddenly changed, one would expect a change in the favored variants in the crystals. However, in the experiments 
there was no observation that would support this concept. The rate of transformation gradually changed to that of the new deformation path. On the other hand, if there might have been a small effect, it would be difficult to capture due to factors related to the design of the setup, such as the stiffness of the machine.

\subsubsection{Reproducibility}

The reproducibility of the tests was investigated by repeating some of the tests a number of times. In Figure 3.11 and 3.12 the results of the repeated tests are presented in $f^{\alpha^{\prime}}-\varepsilon^{\mathrm{eq}}$ graphs for the proportional and the non-proportional tests, respectively. Small deviations were observed only at high strain levels. The reason for this is twofold: at high tensile deformation the sensor starts to be disturbed from its position in the vertical direction and the contact with the specimen becomes loose. Likewise, at high shear deformation the material starts to wrinkle slightly and this causes a disturbance in the contact between the sensor and the sample.

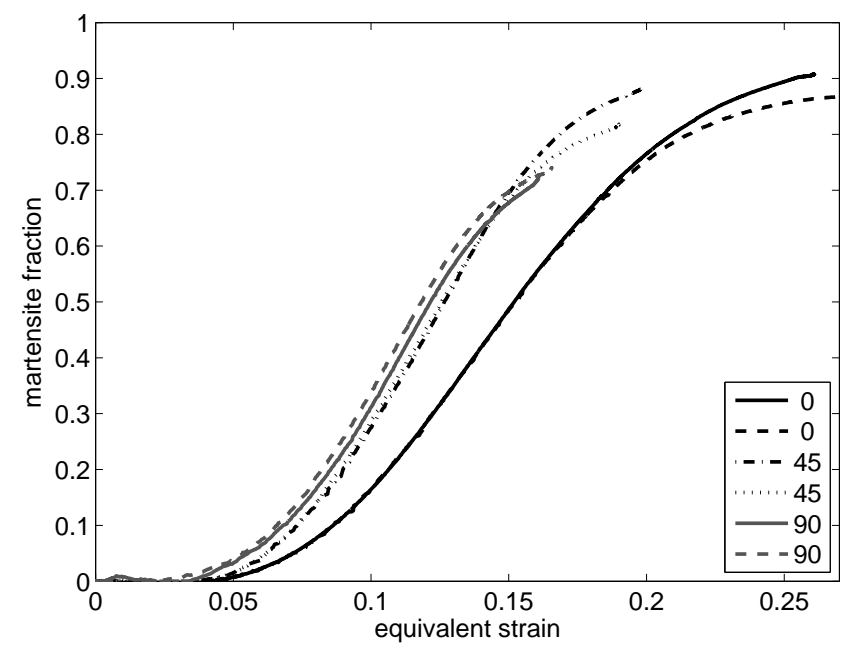

Figure 3.11: Reproducibility of proportional tests: martensite fraction vs. equivalent strain plots for $\alpha$ couples.

The reproducibility of the proportional tests for martensite fraction as well as the stress-strain response was found to be excellent [71]. The non-proportional tests however were found to be less reproducible compared to the proportional tests. The complexity of the deformation paths and the stiffness of the test equipment caused slight deviations in the results. However, it was still possible to observe the effects of the strain-path change qualitatively. 


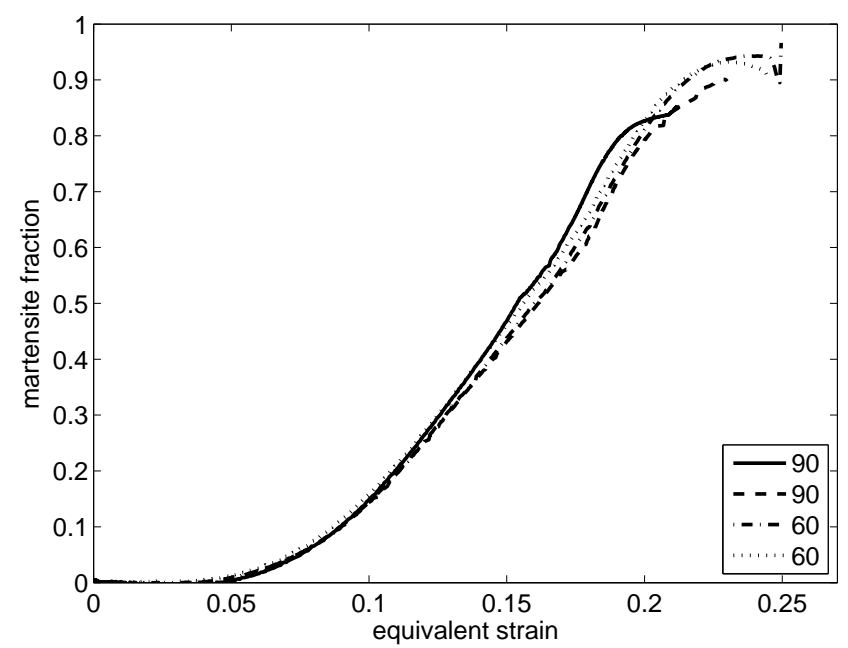

Figure 3.12: Reproducibility of non-proportional tests: martensite fraction vs. equivalent strain plots for $\alpha_{2}$ couples.

\subsection{Effect of strain on transformation kinetics}

Experimentally, it is difficult to observe the effects of plastic strain on the transformation kinetics. It is known that transformation causes considerable inelastic strain when it occurs under stress making it hard to measure the amount of plastic strain (by slip mechanism) in a regular tensile test. Additionally, since the flow stress of the material is dependent on the amount of accumulated plastic strain, it is not possible to attribute the transformation directly to plastic strain based on regular mechanical tests.

Consequently, in order to determine the effect of plastic strain on the transformation, the experiments must fulfill a number of requirements. The amount of plastic strain must be quantifiable; hence, it must be separated from the inelastic strain caused by the transformation. Apart from the difference in accumulated plastic strain, all samples must be deformed under the same conditions to observe the effect of plastic strain only, on the transformation. In the following, an experimental method is proposed by which the above conditions are satisfied.

\subsubsection{Prestrain tests}

The basic idea behind the prestrain experiments was to first plastically strain the material, without causing any transformation, and then perform a tensile test and observe the differences in the transformation behavior of the samples with different accumulated plastic strain. Under normal circumstances this is difficult, since during the initial plastic straining stage the material already starts to transform. Therefore, 
a two stage test setup was planned. The first part of deformation takes place at an elevated temperature motivated by the fact that transformation is affected negatively by a rise in temperature because of the decrease in chemical driving force (see Section 2.1). This was verified by the experiments reported in [74] which show that this material has very slow transformation kinetics at temperatures above $120^{\circ} \mathrm{C}$. The second stage takes place at room temperature where the chemical driving force is strong enough for transformation.

The setup is schematically illustrated in Figure 3.13. The test equipment used in this study was a Zwick Z020 tensile stage placed in a temperature controlled furnace. The sensors used were a Fiedler PS-e100-0153-af Laser extensiometer, an inductive coil to measure permeability and a thermocouple that touches the specimen. The voltage readings from the inductive coil have not been calibrated to actual martensite amount due to the complexities introduced by the test setup. On the other hand, in these tests the relative transformation behavior of samples are considered, therefore in this case the voltage readings are sufficient in the analysis of the results.

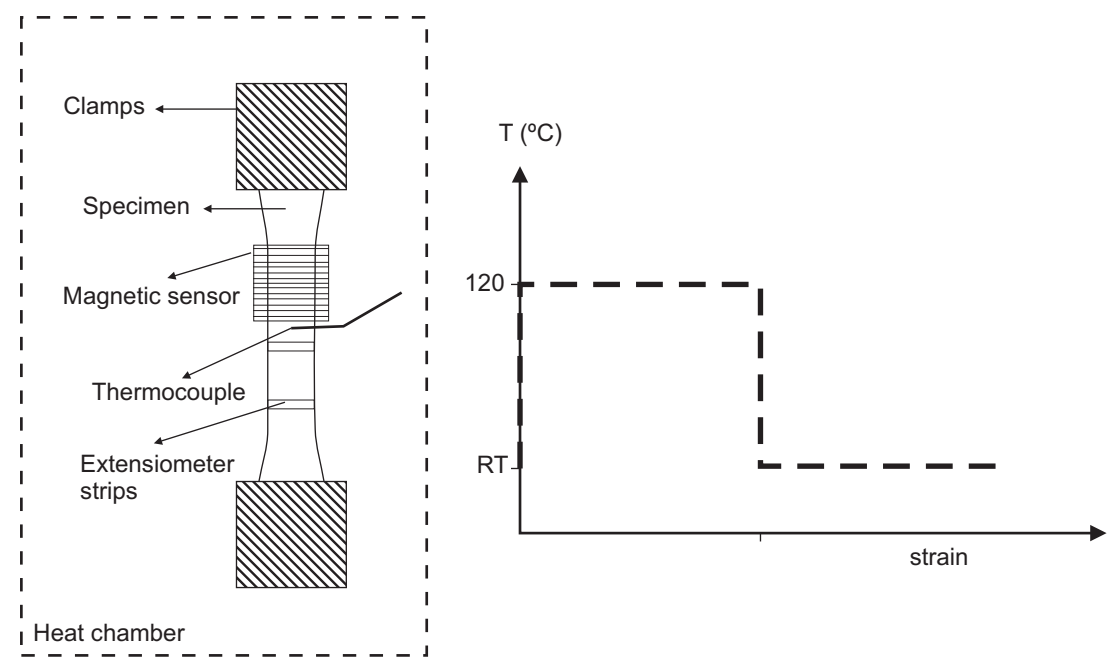

Figure 3.13: Temperature controlled tensile test setup with laser extensiometer (left) and the temperature-strain scheme followed in the experiments (right).

The width, thickness and length of the specimens were approximately $20 \mathrm{~mm}, 0.5 \mathrm{~mm}$ and $120 \mathrm{~mm}$, respectively. The initial gauge length used in the current tests was $50 \mathrm{~mm}$.

The specimens after clamping were heated in the furnace from room temperature $\left(20^{\circ} \mathrm{C}\right)$ to $120^{\circ} \mathrm{C}$ slowly, by approximately $3{ }^{\circ} \mathrm{C} / \mathrm{min}$, and any load that occurred due to expansion was relaxed. Then, at $120^{\circ} \mathrm{C}$ a tensile test was carried out until specified strains were reached after which the specimens were cooled to room temperature in the furnace, with approximately $-3{ }^{\circ} \mathrm{C} / \mathrm{min}$, while continuously unloading the forces due to shrinkage. This procedure allows straining the material plastically without having any significant martensitic transformation. Finally, the tensile test was continued at room temperature. The schematic temperature-strain curve is shown in Figure 3.13. 


\subsubsection{Results}

In Figures 3.14 and 3.15, the stress-strain results of each test during the prestraining stage at $120^{\circ} \mathrm{C}$ and the second stage at $20^{\circ} \mathrm{C}$ are shown, respectively.

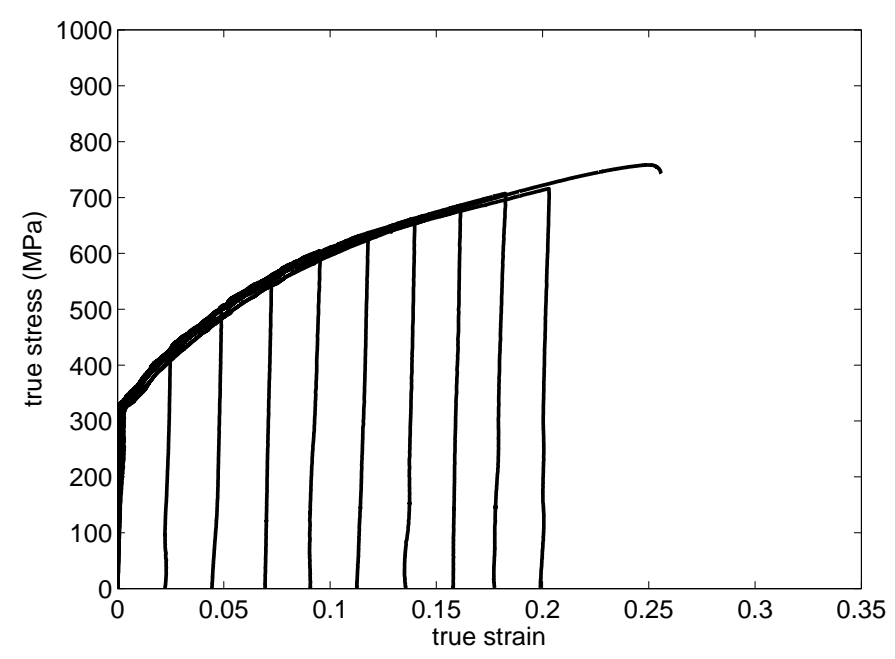

Figure 3.14: Stress-strain curves of the samples during the pre-straining tensile stage at $120^{\circ} \mathrm{C}$.

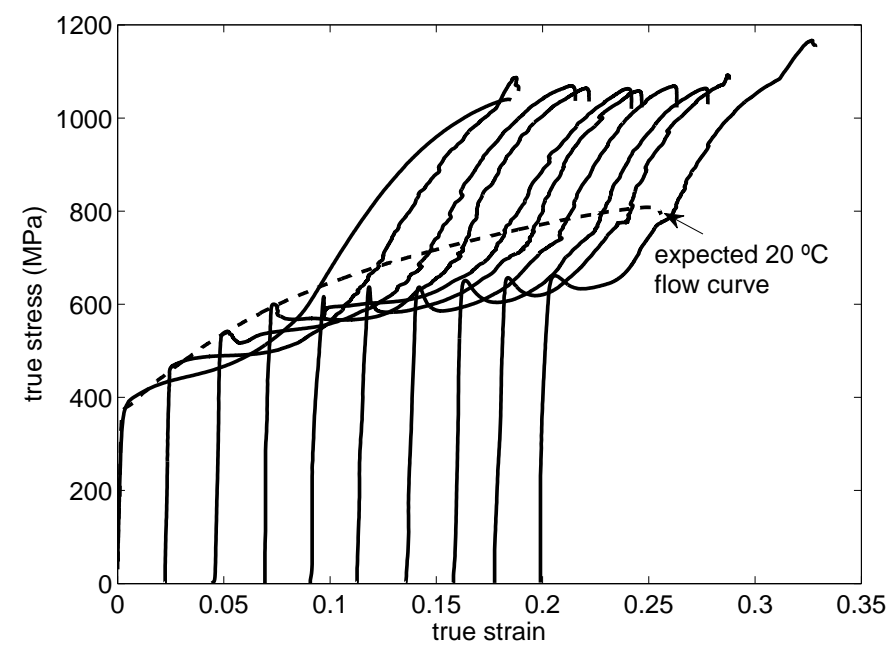

Figure 3.15: Stress-total strain curves of the pre-strained samples during the tensile test at room temperature. 
Figure 3.14 shows that in the first stage, at $120^{\circ} \mathrm{C}$, all samples followed the same hardening behavior. In Figure 3.15 however, it is clear that during the room temperature tests transformation took place. The non-prestrained sample shows the typical mechanical behavior of this steel that was also observed in the Biaxial tests: an initial softening due to transformation induced plasticity followed by a steep hardening due to transition to martensite.

Furthermore, it is seen in Figure 3.15 that samples with different amount of accumulated plastic strain show differences in mechanical behavior. It can be observed that with increasing amount of prestraining the yield stress of the alloy increases. In [74] it is reported that the yield stress of this alloy increases with decreasing temperature. Consequently, the actual yield stress at the room temperature stage is expected to be higher than the one at the $120^{\circ} \mathrm{C}$ stage. This is observed up to around $7 \%$ prestrain. However, beyond this point the material starts to yield at lower stress levels than the $20^{\circ} \mathrm{C}$ flow stress and the yield stress does not increase with increasing prestrain. This suggests that the inelastic strain that causes the yielding of the material is not due to plastic slip but, to transformation induced plasticity, i.e. TRIP. The level of inelastic strain accompanying the transformation corresponds with experimentally observed and theoretically predicted values [57].

In Figures 3.16 and 3.17, the amount of transformation is plotted versus the tensile stress measured during the tests at $120^{\circ} \mathrm{C}$ and at room temperature, respectively. As a starting point, it is clear in Figure 3.17 that within the margins of experimental error, the transformation characteristics of all samples are similar. In all the tests transformation starts distinctively at a certain stress and follows a saturating exponential behavior. With increasing plastic strain, the stress that initiates the transformation is observed to increase.

The theory suggested by Olson and Cohen [68] relates the number of martensitic nucleation sites to plastic strain via shear band intersections. The exact temperature dependency of the creation of shear band intersections with plastic strain is difficult to obtain but it is accepted that shear band formation is retarded at higher temperatures. Therefore, according to the theory, the samples an the high temperature stage cannot transform because not enough nucleation sites are created. At the room temperature stage, for the samples with small amounts of prestrain, the initiation stress of the transformation is found to coincide with the flow stress. This might suggest that the transformation only takes place when the plastic regime is reached since more nucleation sites are created. However, the fact that, for samples with larger prestrains the transformation starts at stress levels below the flow stress, indicates that transformation can start before further plastic deformation. Hence, it is difficult to put the experimental results into the perspective of the strain-induced transformation theory.

An alternative theory could be proposed that relates the transformation directly to the applied stress rather than to the plastic strain. It can be observed that transformation starts at a distinguishable stress level, i.e. critical stress. For each test, the critical stress can be clearly determined from the martensite fraction versus stress curves. This observation supports the stress-driven transformation theory where it is expected that transformation would start as soon as the resolved mechanical driving 


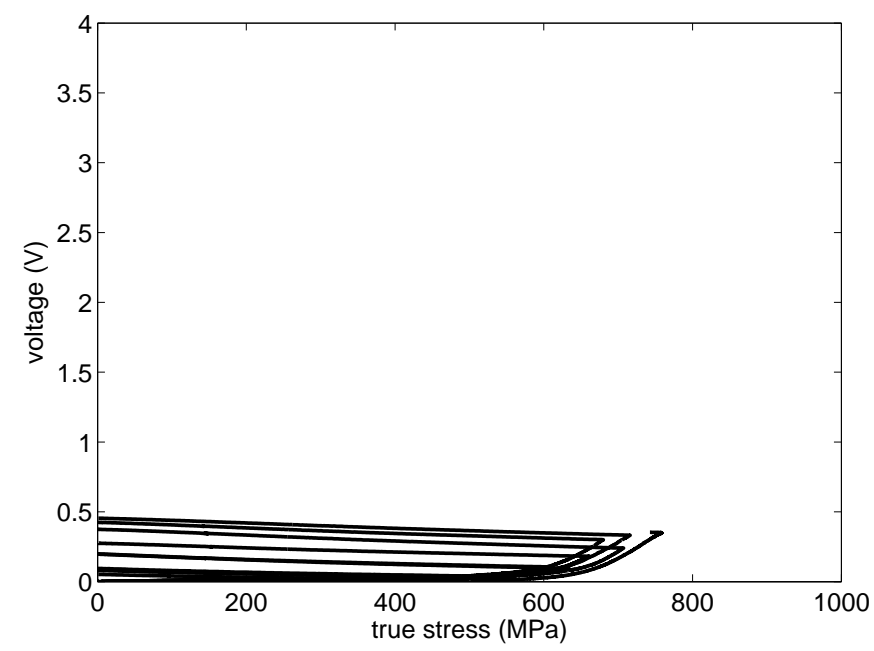

Figure 3.16: Magnetic voltage versus stress curves of the samples during the prestraining tensile stage at $120^{\circ} \mathrm{C}$.

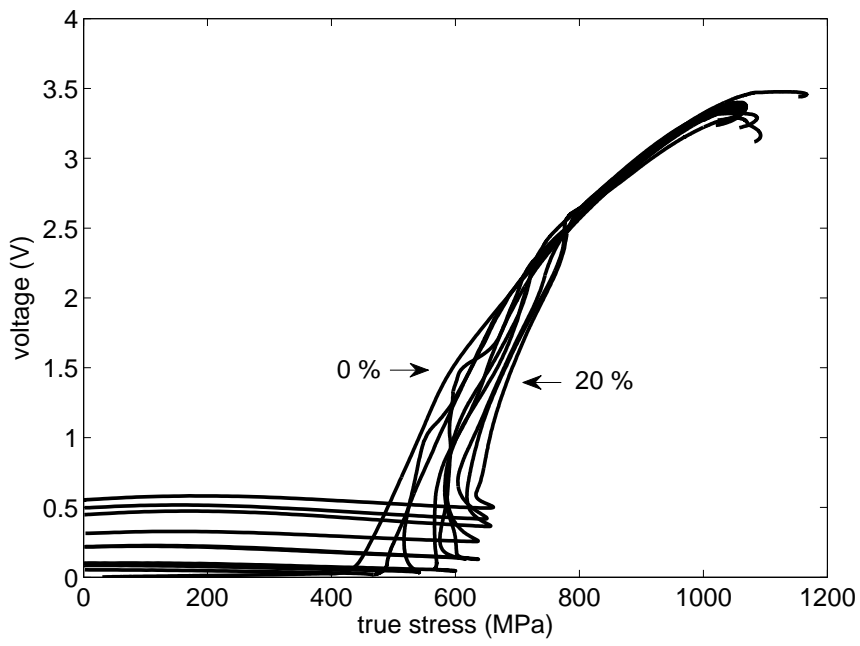

Figure 3.17: Magnetic voltage versus stress curves of the pre-strained samples during the tensile stage at room temperature.

force reaches a critical energy barrier. Furthermore, the results show that once the transformation starts, all the samples follow a similar transformation behavior. This is an interesting result because the largely prestrained samples are not expected to start plastic deformation until the stresses have reached the flow stress, but still they 
show a similar behavior compared to the less prestrained samples.

Following the stress-driven transformation theory, the increase of the critical stress with prestrain can be explained by an increase in the resistance of the matrix to the formation of martensite particles. This can be related to the movement of the glissile transformation interface, as proposed by Maalekian et al. [56].

\subsection{Summary and conclusions}

In this chapter the results of the mechanical tests that were performed in order to broaden the understanding of mechanically induced martensitic transformations were shown.

With the Biaxial tests, the effect of stress on transformation kinetics was analyzed. Austenitic stainless steel samples were deformed under different stress states and the transformation was monitored during the tests. To define the stress states, a unique parameter, i.e. the deformation angle, was used which is a measure of the ratio of tensile and shear deformation. It is observed that with an increasing amount of tension compared to shear, the rate of transformation increases. The strong influence of the stress state on the kinetics emphasizes the role of stress in the mechanically induced transformation phenomenon.

The effect of plastic strain on transformation was investigated with prestrain tests. In these tests, samples of the same material were plastically deformed at an elevated temperature for up to $20 \%$ strain, without any significant transformation. After cooling the specimens down to room temperature, the tensile tests were continued and the transformation was monitored. The samples showed similar transformation behavior once the transformation started. However, an increase in the stress level that initiates the transformation was observed. The strain-induced transformation approach, which is based on the theory that plastic strain is the cause of the transformation, is found to be insufficient for describing the results of the tests. On the other hand, the results support the theory in which transformation is related directly to the action of applied stress.

The results of these experiments are used in the next chapter in which a transformation model is constructed on the basis of these findings. 


\section{Transformation model}

In this chapter a physically based mechanically induced martensitic transformation model is introduced. First, a short introduction and motivation on the methodology is given. Following the introduction, the mechanical driving force acting on a single martensitic variant (see Section 2.2) is formulated. The driving force concept is generalized to a polycrystalline material. Using this formulation, a transformation criterion is proposed that is represented by a transformation 'yield' surface in the principal stress space. The transformation surface is validated using the experimental results presented in the previous chapter. The evolution of martensite fraction is simulated by considering a finite number of randomly oriented grains under increasing stress. The results are compared with the experiments for validation. The meso-scale simulations are used to develop a phenomenological model for the prediction of the transformation based on a stress-driven criterion. Finally, an analytical expression for the transformation plasticity is derived.

\subsection{Introduction}

In the previous chapter, the effects of plastic strain and stress on the transformation were investigated experimentally. The result showed that the effect of stress on the transformation behavior is very significant. It was also noted that a stress-driven theory might describe the physics of the transformation as well as a plastic strain based theory where the latter one is found to be insufficient to explain some of the observations.

The stress-driven approach utilizes the effect of stress on the transformation considering the application of a mechanical driving force on the material $[7,22$, $59,72,85]$. This approach is mostly used in smaller length scales by considering the driving force resolved on each martensitic variant, individually. These studies show that the characteristics of the transformation can be captured without having a direct relation between the transformation and the plastic strain. Since the aim of this study is to develop a macroscale transformation model, microscopic features of 
the transformation must be captured and generalized to obtain an averaged response of the material.

Therefore, the motivation in constructing the transformation model is to generalize the stress-driven transformation theory to macroscale and to define a physical basis with which the evolution of the martensitic transformation can be predicted.

\subsection{Mechanical Driving Force}

As discussed in Section 2.2, during transformation the rearrangement of atoms results in a lattice deformation. The mechanical work done during transformation is a consequence of the interaction of the applied stress with this deformation. This work causes a decrease in the overall free energy in the system; hence it is an additional driving force on the system for transformation [48]. In Figure 4.1, the deformation of a portion of the material during transformation is schematically illustrated. Due to the transformation, the line segment a-b turns into a combination of the partial lines a-c-d-e. In the derivations that follow, for simplicity, the transformation is assumed to take place continuously in space and time. Although thermodynamically the process is known to be irreversible, this assumption does not cause any difference in the resulting formulations.

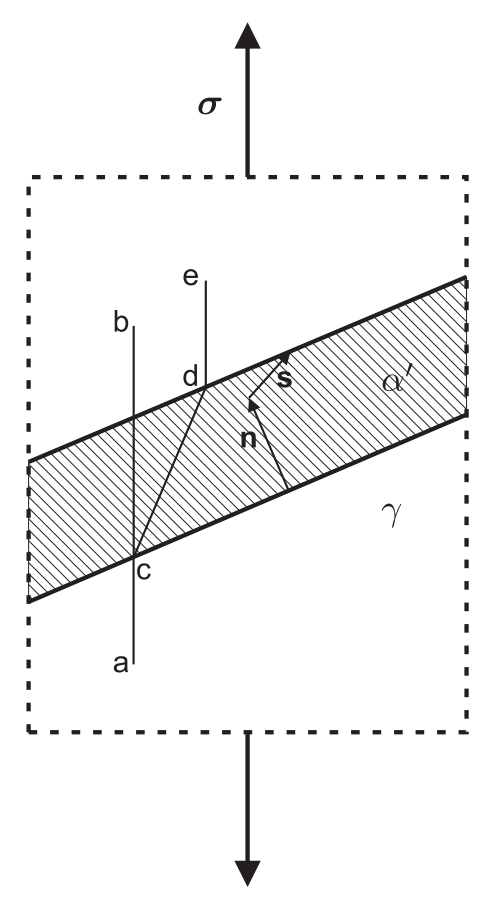

Figure 4.1: The change of the shape of an initial scratch from a-b to a-c-d-e during an unconstrained martensitic transformation. 
In Section 2.2 the deformation gradient associated with the transformation was established as:

$$
\mathbf{F}=\mathbf{I}+\mathbf{s} \otimes \mathbf{n}
$$

where $\mathbf{n}$ and $\mathbf{s}$ are the habit plane normal and the shear direction, respectively.

During the transformation of a variant, the deformation is assumed to take place continuously and the progression of the transformation is defined by a parameter $\xi$ where $0 \leq \xi \leq 1$. This parameter defines the transformed fraction of the total volume that can transform. The deformation gradient of the variant during transformation becomes

$$
\mathbf{F}^{i}=\mathbf{I}+\xi^{i} \mathbf{s}^{i} \otimes \mathbf{n}^{i}
$$

where $i$ is the variant that is transforming. The time derivative of $\mathbf{F}^{i}$ is given by

$$
\dot{\mathbf{F}}^{i}=\dot{\xi}^{i} \mathbf{s}^{i} \otimes \mathbf{n}^{i},
$$

since $\xi$ is the only time dependent parameter in Equation (4.1). Using Equations (4.1) and (4.2) it is possible to calculate the velocity gradient from

$$
\begin{aligned}
\mathbf{L}^{i} & =\dot{\mathbf{F}}^{i} \cdot \mathbf{F}^{i-1}, \\
& =\frac{1}{J^{i}} \dot{\xi}^{i} \mathbf{s}^{i} \otimes \mathbf{n}^{i}
\end{aligned}
$$

where $J^{i}$ is given by:

$$
J^{i}=\operatorname{det} \mathbf{F}^{i}=1+\xi^{i} \mathbf{s}^{i} \cdot \mathbf{n}^{i} .
$$

For a deformation taking place under stress, the mechanical work done is given by

$$
W^{i}=\int_{V} \int_{t} \boldsymbol{\sigma}: \mathbf{L}^{i} \mathrm{~d} t \mathrm{~d} V=\int_{V_{0}} \int_{t} \dot{\mathbf{F}}^{i \mathrm{~T}}: \mathbf{P} \mathrm{d} t \mathrm{~d} V_{0}
$$

where $\mathbf{P}$ is the Nominal stress given by:

$$
\mathbf{P}=J^{i} \mathbf{F}^{i-1} \cdot \boldsymbol{\sigma} .
$$

Combining Equations (4.1) to (4.7) gives:

$$
W^{i}=\int_{V_{0}} \int_{t} \dot{\xi}^{i} \boldsymbol{\sigma}: \mathbf{s}^{i} \otimes \mathbf{n}^{i} \mathrm{~d} t \mathrm{~d} V_{0} .
$$

It is important to note at this point that the unconstrained transformation causes a stress-free deformation (the stress in the material is not dependent on the deformation) and therefore, the stress term in Equation (4.8) is constant. Additionally, $\mathbf{s}^{i}$ and $\mathbf{n}^{i}$ do not change during the transformation which leaves $\xi$ as the only time dependent term in Equation (4.8). Changing the integrand variable from $t$ to $\xi$, Equation (4.8) takes the form

$$
W^{i}=\int_{V_{0}} \int_{0}^{1} \boldsymbol{\sigma}: \mathbf{s}^{i} \otimes \mathbf{n}^{i} \mathrm{~d} \xi \mathrm{d} V_{0},
$$


which, after computing the inner integral becomes,

$$
W^{i}=\int_{V_{0}} \boldsymbol{\sigma}: \mathbf{s}^{i} \otimes \mathbf{n}^{i} \mathrm{~d} V_{0} .
$$

Using this result, the mechanical work density (mechanical work per unit volume) is obtained as:

$$
U^{i}=\boldsymbol{\sigma}: \mathbf{s}^{i} \otimes \mathbf{n}^{i}
$$

and the symmetry of $\boldsymbol{\sigma}$ ensures that

$$
U^{i}=\boldsymbol{\sigma}:\left[\frac{1}{2}\left(\mathbf{s}^{i} \otimes \mathbf{n}^{i}+\mathbf{n}^{i} \otimes \mathbf{s}^{i}\right)\right] .
$$

For convenience, the symmetric part of the deformation tensor will be defined as

$$
\mathbf{T}^{i} \equiv \frac{1}{2}\left(\mathbf{s}^{i} \otimes \mathbf{n}^{i}+\mathbf{n}^{i} \otimes \mathbf{s}^{i}\right)
$$

Equation (4.11) gives the work done by stress during the complete transformation of a martensitic variant per unit volume. The dimension of this term, $\mathrm{J} / \mathrm{m}^{3}$, is consistent with a driving force term. The actual work done will depend on the transformed volume of austenite.

\subsection{Transformation criterion}

From Equation (4.11) it can be deduced that the main factors determining the amount of driving force are the magnitude of the stress and the orientation of the crystal with respect to the stress direction. In a polycrystalline material with no texture, there is a uniform distribution of grain orientations and the stress can be assumed to be uniform across the crystals. This implies that for a given stress magnitude, there will always be a grain which has a martensite variant with the largest mechanical driving force resolved. For this grain and variant, the principal axes of the deformation and the stress coincide. When this is the case, the maximum resolved driving force can be calculated from

$$
U^{\max }=\sum_{j} \sigma_{j}^{*} \lambda_{j}
$$

where $\sigma^{*}$ are the ordered principal stresses and $\lambda$ are the ordered eigenvalues of the T tensor (see Equation (4.13)).

According to Patel and Cohen, the transformation will only start when the maximum mechanical driving force that is resolved in the material reaches a critical value which represents the critical energy barrier:

$$
\left.\Delta G^{\gamma \rightarrow \alpha \prime}\right|_{T}-U=\left.\Delta G^{\gamma \rightarrow \alpha \prime}\right|_{M_{\mathrm{s}}}
$$

Hence, at a certain temperature the critical energy barrier can be assumed to be

$$
\Delta G^{\mathrm{cr}}=\left.\Delta G^{\gamma \rightarrow \alpha \prime}\right|_{T}-\left.\Delta G^{\gamma \rightarrow \alpha^{\prime}}\right|_{M_{\mathrm{s}}} .
$$


The terms on the right hand side in Equation (4.15) are very difficult to evaluate theoretically and experimentally (although for some alloys derivations can be found in the literature $[5,13])$. The objective is to have an estimation of the critical energy barrier and not all the terms in Equation (4.15). Therefore, another approach will be presented below to obtain an estimate of $\Delta G^{\text {cr }}$ at a given temperature.

One requirement for the validity of the stress-driven transformation theory is the occurrence of a critical stress at which transformation starts since the theory suggests that no transformation can occur if the barrier is not reached. Results presented in Figure 3.17 verify that this condition is satisfied since the critical stress can be clearly observed.

Considering that the tensor $\mathbf{T}$ is only dependent on the lattice parameters of austenite and martensite, the only unknown parameters in Equation (4.14) are the principal stresses. Therefore, the critical stress determined from the experiments can be used to estimate the $\Delta G^{\text {cr }}$ using the fact that

$$
\Delta G^{\mathrm{cr}}=\left.U^{\max }\right|_{\sigma=\sigma^{\mathrm{cr}}}
$$

when transformation starts. If a uniaxial stress state is considered, the number of parameters needed to estimate the critical energy barrier is reduced to only one.

Equations (4.14) and (4.16) establish a stress-based criterion for transformation during deformation which can be also visualized as a transformation surface in the principal stress space [24]. The transformation surface is defined by:

$$
f(\boldsymbol{\sigma})=\sum_{j} \sigma_{j}^{*} \lambda_{j}-\Delta G^{\mathrm{cr}}=0 .
$$

\subsubsection{Validation}

The habit planes and shear directions of 12Cr-9Ni-4Mo (ASTM A 564) steel have been calculated using the procedure defined in Section 2.2. The lattice parameters of austenite and martensite were measured by X-ray diffraction as

$$
\begin{aligned}
& a^{\alpha^{\prime}}=2.87351 \AA, \\
& a^{\gamma}=3.59690 \AA .
\end{aligned}
$$

The following are the calculated families of habit planes, $\mathbf{n}$ and shear directions, $\mathbf{s}$ using these lattice parameters:

$$
\begin{aligned}
& \mathbf{n}=\{0.178,0.608,0.774\} \\
& \mathbf{s}=\langle-0.046,-0.156,0.159\rangle \text {. }
\end{aligned}
$$

These planes and directions were used to calculate the eigenvalues of the $\mathbf{T}$ tensor as

$$
\lambda=(-0.1037,0,0.1238) \text {. }
$$

$\Delta G^{\text {cr }}$ can be determined easily using the critical stress values observed in the experiments discussed in the previous chapter. For instance, in the uniaxial tests, 
the measured tensile stress is naturally the third principal stress (according to the ordering used), hence the critical energy barrier is

$$
\Delta G^{\mathrm{cr}}=\lambda_{3} \sigma^{\mathrm{cr}}
$$

where, $\sigma^{\mathrm{cr}}$ is the critical tensile stress.

Using the principal stresses measured at $5 \%$ martensite fraction for the biaxial and uniaxial tests, the critical energy barrier was calculated to be $60 \mathrm{MPa}(7.1 \mathrm{~J} / \mathrm{g}$ or $400 \mathrm{~J} / \mathrm{mole}$ ). The transformation surface using this value for the energy barrier and the experimentally determined points are plotted on the principal stress space in Figure 4.2. It is clear from the figure that the results are in good correspondence with the experiments. It is observed that the surface has sharp corners at the uniaxial and the biaxial tension points, resembling the Tresca yield surface. Furthermore, the area in compression is noticeably larger than that in tension due to the hydrostatic stress dependence of the transformation. The same type of transformation surface has been predicted by Olson and Cohen [69] for the transformation at stress levels below the yield stress. It is interesting to see that the same criterion holds in the transformation taking place in the plastic regime.

The small deviations can be related to the difference in the critical energy barrier due to chemical and processing variations in the material. Additionally, the uniform stress assumption might also play a role on the deviations since the stress resolved in a crystal can be slightly different from the applied stress on the material.

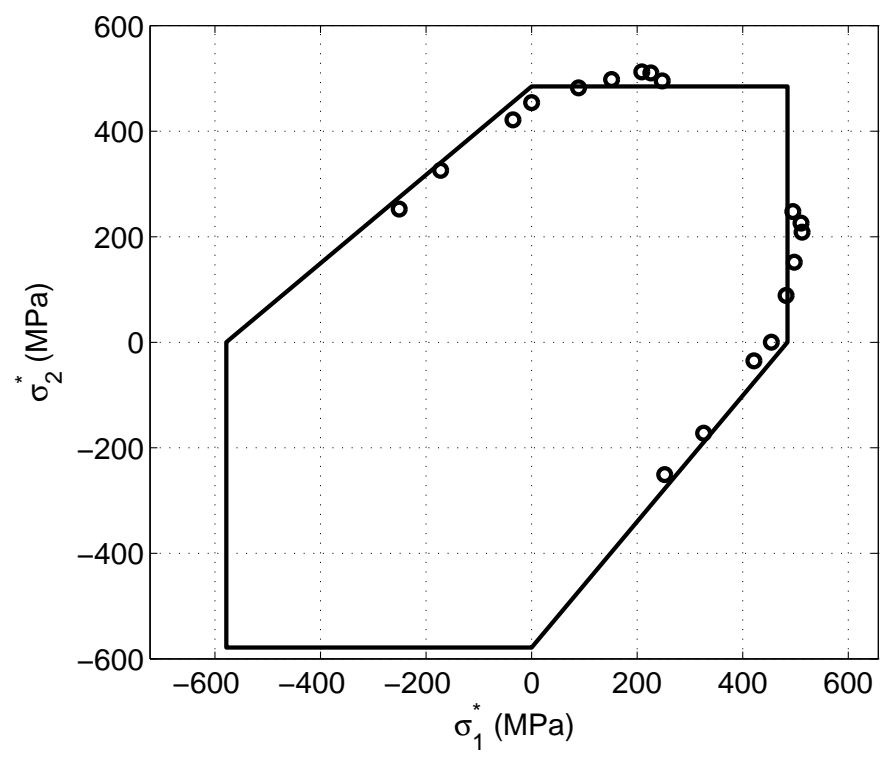

Figure 4.2: Measured principal stresses at 0.05 martensite and the calculated transformation surface for $\Delta G^{\mathrm{cr}}=400 \mathrm{~J} / \mathrm{mole}$. 
The calculated transformation surface in three dimensional principal stress space is shown in Figure 4.3. The shape of the surface is similar to a Mohr-Coulomb type yield surface. Additionally, since the sum of the eigenvalues does not vanish, hydrostatic pressure plays a role on the width of the hexagon. The surface converges to a single point on the hydrostatic stress axis at a stress level of $3000 \mathrm{MPa}$. This suggests that transformation can be mechanically induced by a pure hydrostatic stress state.
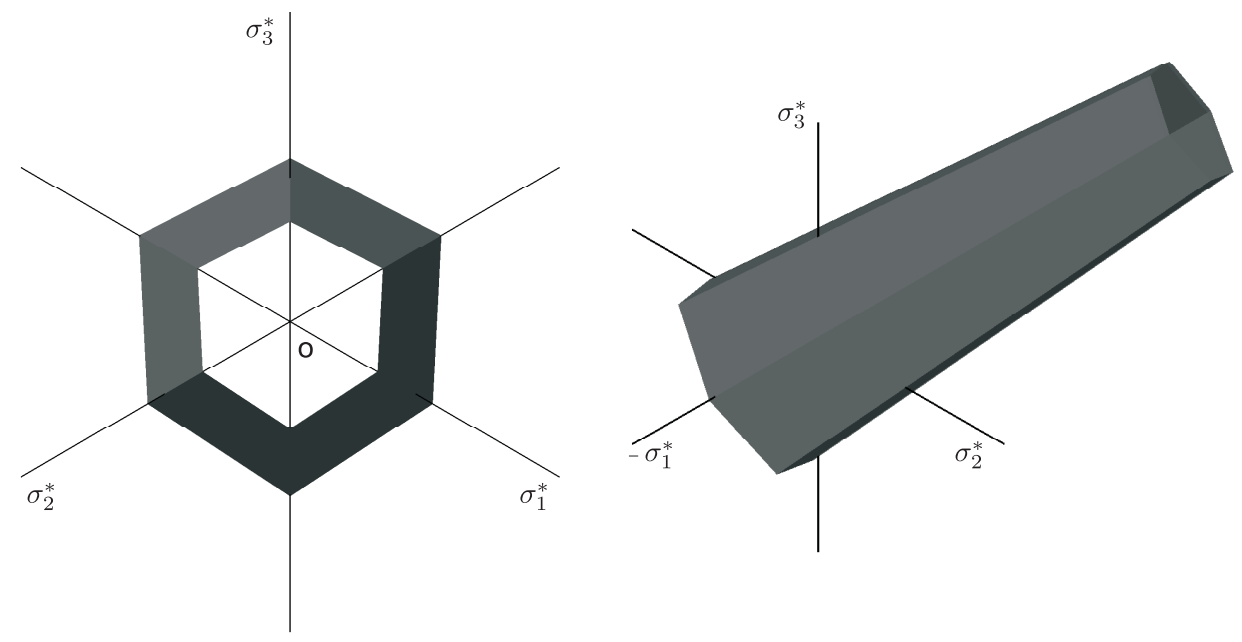

Figure 4.3: Calculated transformation surface in three dimensional principal stress space.

\subsection{Evolution of martensite fraction}

The mechanical driving force for a given grain orientation was calculated by Equation (4.12). The effect of crystal orientation in this equation lies in the selection of the coordinate basis for the stress or the $\mathbf{T}$ tensor. Randomly rotating tensor $\mathbf{T}$ a finite number of times, and assuming once again a uniform stress distribution among grains as well as an equal volume of transformation for each grain and variant, gives the following distribution of the driving force throughout the material:

$$
U^{i}(\boldsymbol{\sigma}, \mathbf{R})=\boldsymbol{\sigma}: \mathbf{R} \cdot \mathbf{T}^{i} \cdot \mathbf{R}^{\mathrm{T}} .
$$

where, $i$ represents the martensitic variants in the crystal and $\mathbf{R}$ is a rotation matrix operating on the crystal orientation. Similar approaches have been followed for shape memory alloys by Huang [36] and Lexcellent et al. [52]. Hallberg et al. [27] applied the same method to austenitic stainless steels for the determination of the transformation surface. 
Equation (4.22) gives the driving force resolved on each variant in each grain. It is obvious that within the grain the driving force will vary among the variants. Some of them may have the potential to transform and some may not. Hence, it is difficult with this knowledge to comment on which of the variants will transform. The selection of the variants to transform may lead to different results depending on the selection criterion [47]. On the other hand, it is clear that if the maximum driving force resolved in a grain is above the energy barrier, transformation will take place. By optical microscopy, the transformation of grains under stress has been investigated in [60]. The results of this study support the assumption that the potential of a grain to transform can be judged by considering the variant with the maximum $U$. Consequently, a simpler distribution of $U$ is introduced as:

$$
U(\boldsymbol{\sigma}, \mathbf{R})=\max _{i}\left\{\boldsymbol{\sigma}: \mathbf{R} \cdot \mathbf{T}^{i} \cdot \mathbf{R}^{\mathrm{T}}\right\} .
$$

Equation (4.23) reflects the fact that the distribution is affected by the stress-state. The maximum driving force, on the other hand, depends only on the invariants of the stress tensor hence will be equal to the one predicted by Equation (4.14).

Equation (4.23) supplies information on the magnitude of the resolved driving force on a variant but lacks information on the actual volume of austenite that would transform. It is clear that in order to be able to compute the actual work done by transformation, the transforming volume must be known. Consequently, at this point another assumption has to be made. The transforming volume of austenite depends on microstructural factors, such as grain size. For simplicity, however, in the evolution model every grain is assumed to have the same transformation volume.

\subsubsection{Validation}

In order to simulate the transformation of a polycrystalline austenitic steel, a representative material with a finite number of grains was chosen. The grain orientations were simulated by rotating all the variants in a crystal with a random rotation in 3-D space. The homogeneity of the rotations is illustrated in Figure 4.4 by $\left\langle\begin{array}{lll}1 & 0 & 0\end{array}\right\rangle$ and $\left\langle\begin{array}{lll}1 & 1 & 0\end{array}\right)$ pole figures of 1000 crystallites.
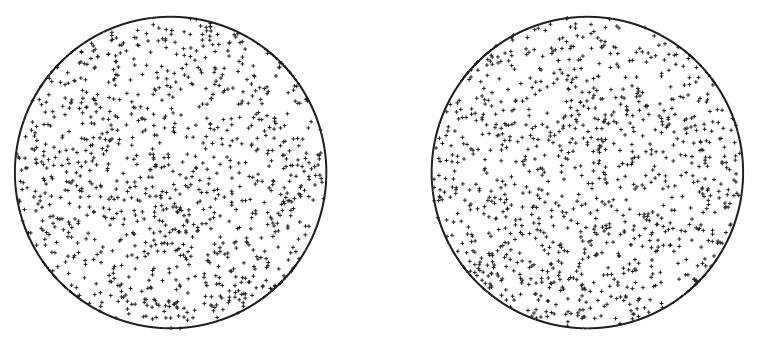

Figure 4.4: $\left\langle\begin{array}{llll}1 & 0 & 0\end{array}\right\rangle$ (left) and $\left\langle\begin{array}{llll}1 & 1 & 0\end{array}\right.$ (right) equal area pole figures of 1000 crystallites. 
Having obtained the rotated variant orientations, $U(\boldsymbol{\sigma}, \mathbf{R})$ can be calculated for any given stress tensor. Figures 4.5 and 4.6 show the resulting distributions in 10000 crystallites for 2 different stress tensors with the components given as:

$$
\left[\boldsymbol{\sigma}^{\mathrm{a}}\right]=\left[\begin{array}{lll}
1 & 0 & 0 \\
0 & 0 & 0 \\
0 & 0 & 0
\end{array}\right], \quad\left[\boldsymbol{\sigma}^{\mathrm{b}}\right]=\left[\begin{array}{ccc}
0 & 1 & 0 \\
1 & 0 & 0 \\
0 & 0 & 0
\end{array}\right]
$$

where $\sigma^{\mathrm{a}}$ and $\boldsymbol{\sigma}^{\mathrm{b}}$ represent uniaxial tension and simple shear stress states, respectively.

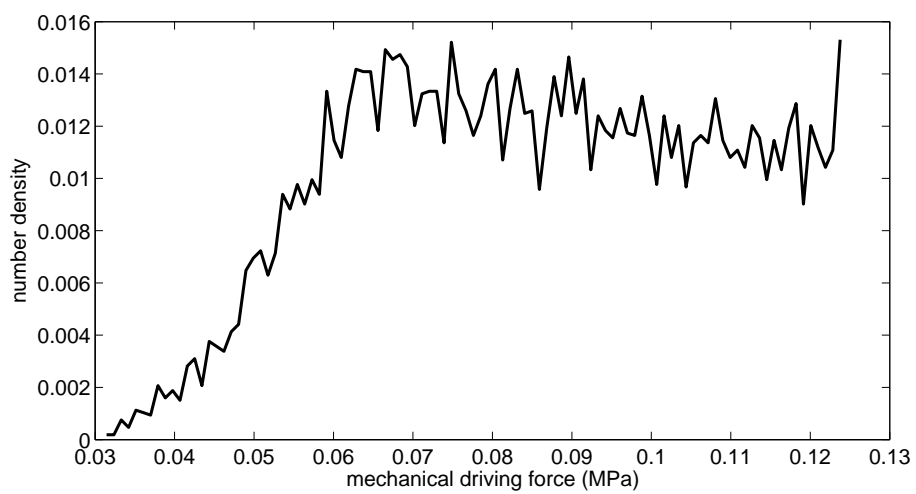

Figure 4.5: Number density vs. resolved driving force plot for stress tensor $\boldsymbol{\sigma}^{\mathrm{a}}$; uniaxial tension.

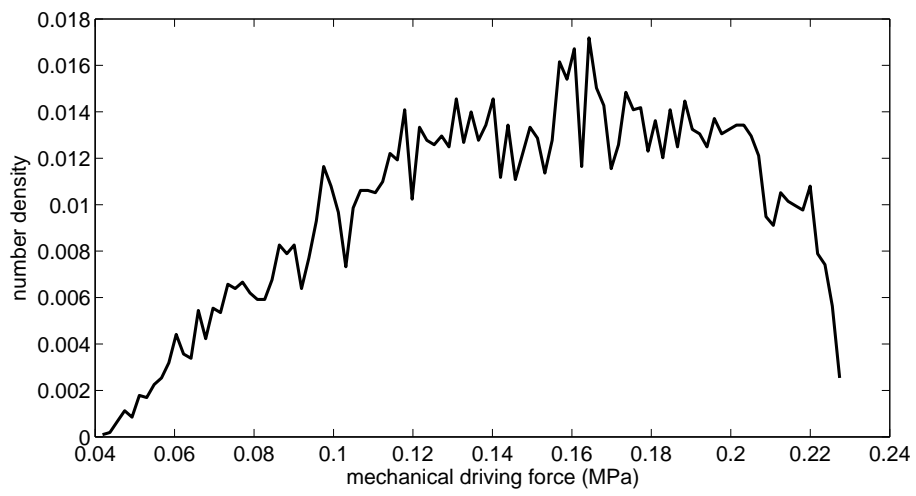

Figure 4.6: Number density vs. resolved driving force plot for stress tensor $\boldsymbol{\sigma}^{\mathrm{b}}$; simple shear.

In Figures 4.5 and 4.6, the maximum resolved driving forces are 0.1238 and 0.2275 which are consistent with Equation (4.14) for the eigenvalues supplied in (4.20). 
To observe the evolution of martensite fraction a simple simulation was performed where, a certain energy barrier was assumed (58 MPa) and the magnitude of the stress ws increased, keeping its direction constant. After each increment of stress, the fraction of the grains with the potential to transform were calculated. The transformed volume fraction was determined by making use of the equal volume assumption.

Although the model in this state is very simple, this simulation is important in qualitatively verifying the stress-driven transformation theory. The results of the simulation for uniaxial tension and simple shear are compared to the experimental results in Figure 4.7.

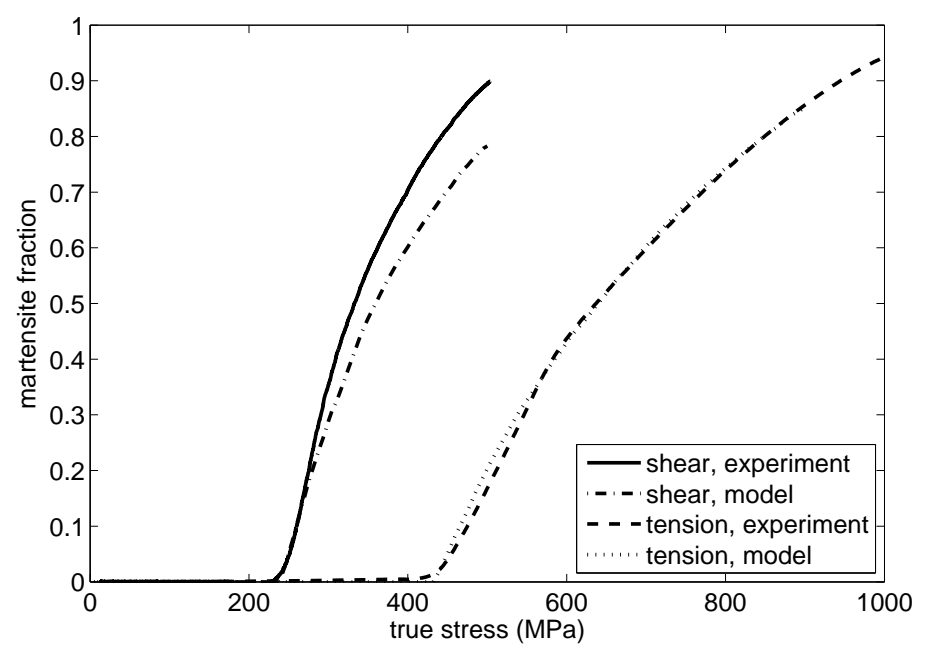

Figure 4.7: Results of the model for simple shear and uniaxial tension stress states and comparison with experiments.

Figure 4.7 shows that qualitatively the model is able to capture the main characteristics of the transformation. As expected, until a critical stress level there is no transformation and after the critical energy barrier is reached, the austenite grains start transforming. It is an important outcome of this model that to increase the martensite volume fraction, the resolved driving force must be increased. Hence, plastic strain plays the important role of hardening the austenite phase thus making it possible to resolve more stress.

One important point to mention is that the model is based on the stress that is concentrated on austenite grains, whereas in the experiments, the measured stress is the overall stress of the austenite-martensite composite. Since there is a big contrast in the mechanical behavior of the two phases, the overall stress is expected to deviate from the austenite stress after some amount of martensite has formed. On the other hand, at the initial stages of transformation the two stresses are expected to be approximately equal. Considering this factor it can be concluded that the 
model underestimates the transformation. There are a number of missing factors in the model such as the ambiguity of the transformed volume and selection of the transforming variants that can cause this discrepancy.

\subsubsection{Phenomenological extension}

The plots presented in Figure 4.7 are dependent on the type of the performed test. However, in a more general way the driving force term defined in Equation (4.14) can be used as an effective stress measure and the experimental results can be plotted using that measure as in Figure 4.8 .

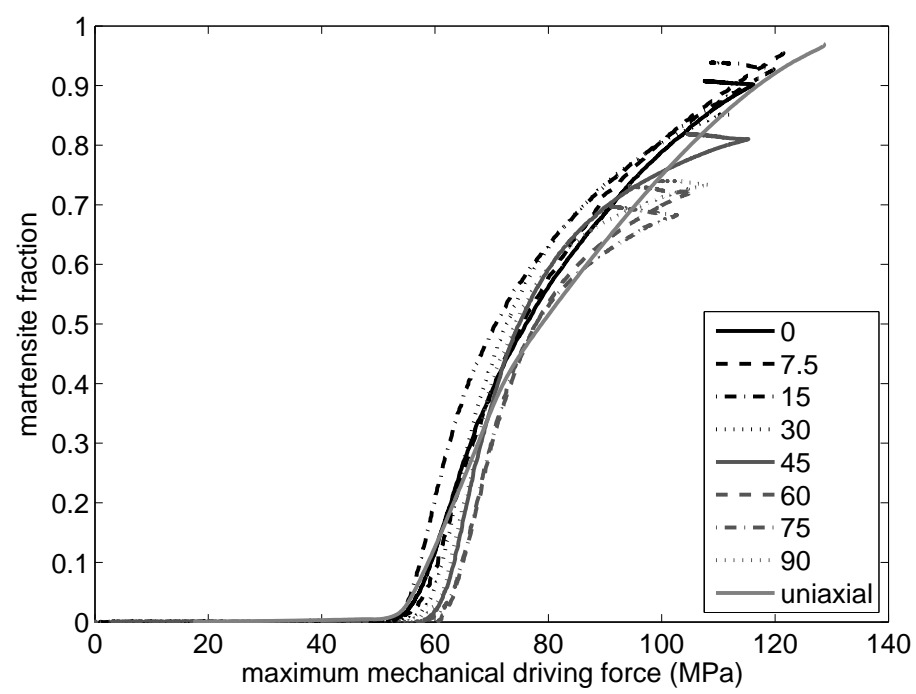

Figure 4.8: Martensite fraction vs. maximum resolved driving force curves for the biaxial and uniaxial experiments.

It is seen in Figure 4.8 that within the experimental error, the evolution of the martensite fraction with maximum mechanical driving force shows a similar behavior for all the tests. Small differences are observed in the critical energy barrier which is basically a repetition of the deviations seen in the transformation surface diagram in Figure 4.2 .

A phenomenological expression is proposed which is based on the results shown in Figure 4.8. The aim is to define a function which will determine, for a given stress tensor, how much martensite is expected to form. The proposed expression is of the form

$$
f^{\alpha^{\prime}}=1-\left[1+(r-1)\left(\frac{U^{\max }}{\Delta G^{\mathrm{cr}}+\Delta G^{\text {off }}}\right)^{m}\right]^{\frac{1}{1-r}}
$$


where $m, r$ and $\Delta G^{\text {off }}$ are fitting parameters, $U^{\max }$ is calculated by Equation (4.14) and $\Delta G^{\mathrm{cr}}$ is the critical energy barrier determined either experimentally or theoretically. A similar evolution function has been used in [27] with a different effective stress measure.

\subsection{Transformation plasticity}

According to Magee [57], the inelastic deformation in martensitic transformations is mainly due to the selective transformation of transforming variants under stress. Hence, in the absence of stress there will be no plasticity due to transformation (Greenwood-Johnson [26] mechanism is assumed to have a small effect compared to the Magee mechanism)(see Section 2.4). Under applied stress however, the net strain will always be in the deviatoric stress direction $[57,80]$. Consequently, the transformation deformation rate can be represented as

$$
\mathbf{D}=\dot{f}^{\alpha^{\prime}}\left(T \mathbf{N}+\frac{\delta V}{3} \mathbf{I}\right)
$$

where $T$ is a scalar that defines the amount of shape change, $\delta V$ is the volume change, $\mathbf{N}$ is the deviatoric stress direction given by

$$
\mathbf{N}=\frac{\boldsymbol{\sigma}^{\prime}}{\sqrt{\boldsymbol{\sigma}^{\prime}: \boldsymbol{\sigma}^{\prime}}}
$$

and $\boldsymbol{\sigma}^{\prime}$ is the deviatoric part of the stress tensor.

Equation (4.26) shows that to completely define the transformation plasticity, it is sufficient to determine the shape change parameter $T$, which gives the magnitude of the deviatoric strain in the deviatoric stress direction.

One of the outputs of the multi-grain simulations presented in the last section is the deformation gradient of the variants that were allowed to transform. Using the deformation gradients, the transformation deformation rate at each stress increment can be calculated as the average deformation rate of the transforming orientations. Once the incremental deformation rate is known, the shape change parameter $T$ can be calculated.

The total deformation rate is the average of deformation rates caused by all transforming variants:

$$
\mathbf{D}=\dot{f}^{\alpha^{\prime}} \frac{1}{n} \frac{1}{J} \sum_{i=1}^{n} \mathbf{T}^{i}
$$

where $n$ is the number of transforming variants in the current step. Finally, Equations (4.28) and (4.26) show that $T$ can be obtained by projecting $\mathbf{D}$ on $\mathbf{N}$ as

$$
T=\frac{1}{n} \frac{1}{J} \sum_{i=1}^{n} \mathbf{T}^{i}: \mathbf{N}
$$


The results of the simulation are presented in Figure 4.9 for shear and tensile stress states. It is clearly seen that the crystals that transform early have much more contribution to the transformation plasticity than those that transform later. This is an expected result since the favorability of a grain is proportional to the alignment of the transformation deformation with the stress direction. The results are in good agreement with the experimental findings in terms of the total transformation strain $[57]$.

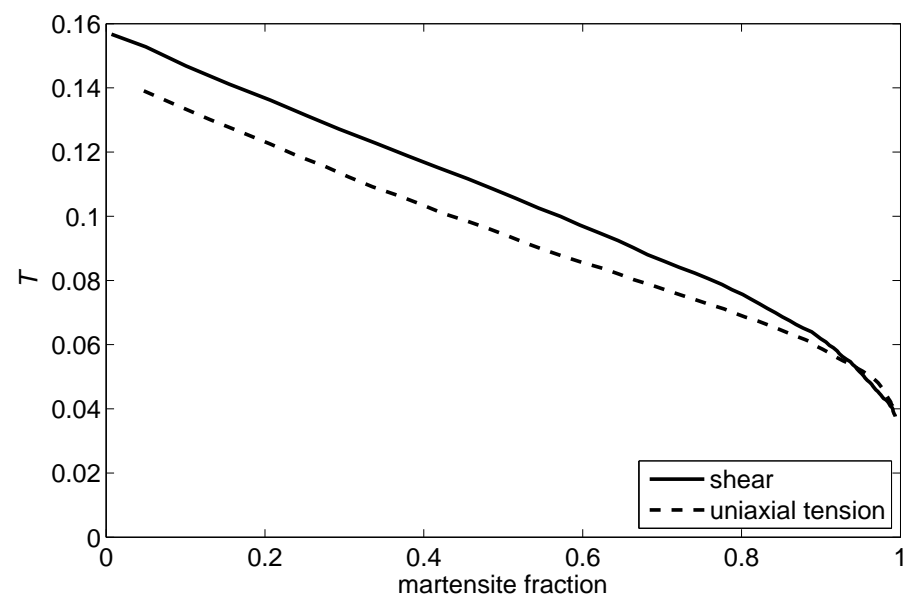

Figure 4.9: Calculated shape change parameter, $T$, vs. martensite fraction curves for two different stress states.

To use in the constitutive model, along with the phenomenological transformation model, an alternative calculation of the transformation strain is necessary. The following derivation results in an analytical expression for the shape change coefficient $T$.

The maximum shape change that can occur with transformation is when the orientation of the crystal is the one that gives maximum driving force, $U^{\text {max }}$. As proposed earlier this is the orientation which has the same principal axes of the $\mathbf{T}$ tensor and $\boldsymbol{\sigma}$. Hence:

$$
T^{\max }=\frac{1}{J} \sum_{j} N_{j}^{*} \lambda_{j}
$$

where $N^{*}$ are the eigenvalues of the deviatoric stress direction (see Equation (4.27)). Using Equation (4.26), for a given stress, the maximum resolved driving force is calculated as

$$
U^{\max }=J \boldsymbol{\sigma}:\left(T^{\max } \mathbf{N}+\frac{\delta V}{3} \mathbf{I}\right),
$$

whereas the transforming variants under that stress need only $\Delta G^{\text {cr }}$ which is given by

$$
\Delta G^{\mathrm{cr}}=J \boldsymbol{\sigma}:\left(T \mathbf{N}+\frac{\delta V}{3} \mathbf{I}\right) .
$$


Since $U^{\max }$ and $\Delta G^{\mathrm{cr}}$ are both known, $T$ can be obtained by the following:

$$
\frac{U^{\max }}{\Delta G^{\mathrm{cr}}}=\frac{J \boldsymbol{\sigma}:\left(T^{\max } \mathbf{N}+\frac{\delta V}{3} \mathbf{I}\right)}{J \boldsymbol{\sigma}:\left(T \mathbf{N}+\frac{\delta V}{3} \mathbf{I}\right)}
$$

which after some algebra yields:

$$
T=\frac{\Delta G^{\mathrm{cr}}}{U^{\max }} T^{\max }+\left(\frac{\Delta G^{\mathrm{cr}}}{U^{\max }}-1\right) \frac{\delta V}{3} \frac{\boldsymbol{\sigma}: \mathbf{I}}{\boldsymbol{\sigma}: \mathbf{N}} .
$$

\subsection{Summary and conclusions}

In view of the experimental results presented in the previous chapter, a transformation model was constructed which is based on the theory that applied stress is the main driving factor for the transformation. The resolved stress acts as a source of additional driving force on the material for transforming austenite to martensite. Therefore, a definition for the mechanical driving force was established and it was shown that the driving force is not a single value but a distribution when a polycrystalline material is considered.

A transformation criterion was developed that can successfully predict the onset of transformation in principal stress space, and can be thought of as a transformation 'yield' surface. A good agreement with the experimental results was observed which supports the stress-driven transformation approach.

The evolution of martensite fraction was simulated by integrating the fraction of favored crystal orientations under a certain applied stress. A qualitative agreement with experiments was obtained by a simulation using only one parameter which defines the critical energy barrier. In the experimental results, a general and consistent behavior of martensite fraction evolution against the resolved maximum mechanical driving force was observed. Hence, a phenomenological expression was proposed using the driving force as the independent variable on the transformation.

Using the martensite evolution simulations, transformation plasticity according to Magee mechanism was calculated and found to be consistent with experimental findings. An analytical expression for the transformation strain was also derived to be used in the constitutive model. 


\section{Homogenization of elastic-plastic composites}

It was discussed in the previous chapters that metastable austenitic stainless steels, by their nature, consist of two different phases with different physical and chemical properties. Austenite, the parent phase, has higher density, lower yield stress and higher strain hardening compared to the product phase, martensite. During deformation as austenite gradually transforms to martensite, the mechanical properties of the composite also changes. In order to simulate the process physically, it is necessary to compute the mechanical behavior when the two phases coexist in the material.

Homogenization, in mechanics, is defined as getting a homogenized response of a material with inhomogeneities. Generally, most of the common materials are inhomogeneous. However, in macroscale, they show a reproducible and homogeneous response which usually makes the continuum approximation valid. In other cases where the microstructure of the material is evolving or more detailed information in smaller scales is required, the continuum approach is usually not sufficient.

Among the most common homogenization methods are Direct Finite Element calculations in micro-scale and Mean-Field methods. The Direct Finite Element method is more accurate and comprehensive as it not only provides the average fields in the constituent phases, but also the local distribution of microfields. Unfortunately, the numerical computations are very demanding which limits the applicability of this method in macroscale applications. The Mean-Field methods are generally faster compared to other methods and supply information on the average fields in the constituent phases as well as the homogenized response of the composite.

In this chapter, first, an introduction to the Mean-Field methods is given that briefly discusses the general theories on scale transition methods. Following that, the early work on homogenization of elastic composite materials are summarized and the well-known Eshelby theories are elaborated. Different schemes that are used in the homogenization algorithms are addressed, followed by the generalization of the 
homogenization theory to elastic-plastic composite materials. Finally, the results of elastic-plastic simulations are presented and discussed.

\subsection{General concepts in homogenization}

Generally, in macroscale, materials are observed to have homogeneous mechanical properties. However, at lower length scales numerous heterogeneities exist in the material, such as different phases, grain boundaries, dislocations, voids, etc. To have a better understanding of the behavior of some materials in macroscale, it is necessary to take into account these heterogeneities when for instance when a composite material is under consideration.

For such cases, Hill [32] introduced the concept of a representative volume element (RVE). An RVE is an equivalent representation of a macroscopic point in the material (i.e. an integration point in a Finite Element model) with a finite inhomogeneous volume that is representative for the microstructure of the material. Therefore, by a consistent transition between the scales, it is possible to solve a complementary problem on the RVE and then use the results in macroscale. This is schematically illustrated in Figure 5.1.

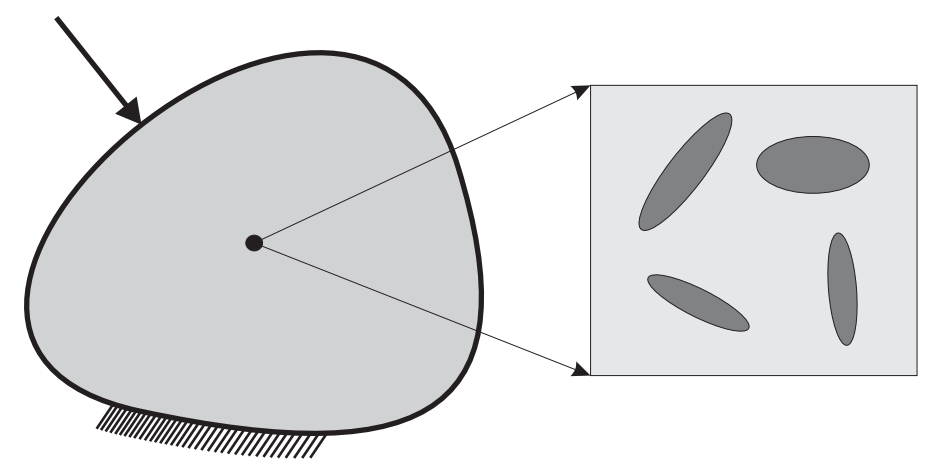

Figure 5.1: Schematic illustration of the scale transition. A macroscopic material point is represented by a finite volume (RVE) that has information on the microstructure of the material.

A Direct Finite Element method for homogenization uses the RVE concept as defined above and for each integration point, the macroscale variables are transferred to the RVE in terms of boundary conditions and a FE solution of the boundary value problem is obtained $[44,45]$. The results are then averaged over the RVE and returned to macroscale. This procedure is very accurate since a detailed analysis of the stresses and strains that develop in the microscale is known. As mentioned earlier, it is clear that this method is computationally very expensive.

The Mean-Field method is also built on the concept of an RVE; however instead of a numerical solution, an analytical estimation of the problem is carried out at the 
smaller scale [16]. Mainly Mean-Field algorithms differ in the choice of the analytical estimation.

\subsubsection{Scale transition}

The scale transition problem deals with the averaging of the fields in the RVE and then transferring the averaged results to macroscale. This is achieved by an averaging operator defined as:

$$
\bar{g}=\langle g(\mathbf{x})\rangle_{\omega}=\frac{1}{V} \int_{\omega} g(\mathbf{x}) \mathrm{d} V
$$

where $g$ is any property, $\bar{g}$ is the macroscopic value and $\langle g(\mathbf{x})\rangle_{\omega}$ is the averaged value of $g$ over the coordinates $\mathbf{x}$ in the RVE volume $\omega$.

When the averaging operator acts on strain and stress and the divergence theorem is applied, the following important equations are obtained:

$$
\begin{aligned}
& \bar{\varepsilon}=\langle\varepsilon(\mathbf{x})\rangle_{\omega}=\frac{1}{V} \int_{\omega} \varepsilon(\mathbf{x}) \mathrm{d} V=\frac{1}{2 V} \int_{\Gamma}(\mathbf{u} \otimes \mathbf{n}+\mathbf{n} \otimes \mathbf{u}) \mathrm{d} A, \\
& \overline{\boldsymbol{\sigma}}=\langle\boldsymbol{\sigma}(\mathbf{x})\rangle_{\omega}=\frac{1}{V} \int_{\omega} \boldsymbol{\sigma}(\mathbf{x}) \mathrm{d} V=\frac{1}{V} \int_{\Gamma} \mathbf{t} \otimes \mathbf{x} \mathrm{d} A
\end{aligned}
$$

where $\Gamma$ defines the boundaries of the RVE, $\mathbf{n}$ and $\mathbf{t}$ are the normal and the traction on the boundaries, respectively.

\subsubsection{Hill's lemma}

To be able to justify Equations (5.2) the mechanical work in both scales must be consistent. This requires that the averaged mechanical work over the RVE is equal to the mechanical work that is calculated using the averaged stress and strain. In mathematical terms:

$$
\bar{\sigma}: \bar{\varepsilon}=\langle\boldsymbol{\sigma}\rangle:\langle\varepsilon\rangle=\langle\boldsymbol{\sigma}: \varepsilon\rangle .
$$

Equation (5.3) is known as the Hill-Mandel condition and is valid in all cases provided that equilibrium and compatibility within the RVE are satisfied [32].

\subsection{Homogenization of elastic composites}

In this section, Mean-Field homogenization of linear elastic two-phase composite materials is discussed. In a two-phase composite material, usually, the phase with the higher volume fraction is referred to as matrix and the second phase particles are referred to as inclusions. The main difference between the two phases therefore lies in the geometry of the particles. The matrix phase is continuous and interconnected whereas the inclusions are particles apart from each other. Intuitively it is imagined that this only holds for low volume fraction of inclusions since for higher volume fractions the particles will start touching each other and form interconnections. 
An RVE, composed of two phases, can be subdivided into two volumes representing each phase and Equation (5.1) can be rewritten utilizing this decomposition. The macroscopic stress becomes,

$$
\begin{aligned}
\overline{\boldsymbol{\sigma}} & =\frac{1}{V} \int_{\omega} \boldsymbol{\sigma}(\mathbf{x}) \mathrm{d} V=\frac{1}{V}\left(\int_{\omega_{0}} \boldsymbol{\sigma}(\mathbf{x}) \mathrm{d} V+\int_{\omega_{1}} \boldsymbol{\sigma}(\mathbf{x}) \mathrm{d} V\right) \\
& =\frac{V_{0}}{V}\langle\boldsymbol{\sigma}\rangle_{\omega_{0}}+\frac{V_{1}}{V}\langle\boldsymbol{\sigma}\rangle_{\omega_{1}}=f_{0}\langle\boldsymbol{\sigma}\rangle_{\omega_{0}}+f_{1}\langle\boldsymbol{\sigma}\rangle_{\omega_{1}}
\end{aligned}
$$

where $f$ denotes the volume fraction of a phase with subscripts 0 and 1 denoting the matrix and inclusion phases, respectively. Clearly,

$$
f_{0}+f_{1}=1
$$

The same holds for strain:

$$
\begin{aligned}
\bar{\varepsilon} & =\frac{1}{V} \int_{\omega} \varepsilon(\mathbf{x}) \mathrm{d} V=\frac{1}{V}\left(\int_{\omega_{0}} \varepsilon(\mathbf{x}) \mathrm{d} V_{0}+\int_{\omega_{1}} \varepsilon(\mathbf{x}) \mathrm{d} V_{1}\right) \\
& =\frac{V_{0}}{V}\langle\varepsilon\rangle_{\omega_{0}}+\frac{V_{1}}{V}\langle\varepsilon\rangle_{\omega_{1}}=f_{0}\langle\varepsilon\rangle_{\omega_{0}}+f_{1}\langle\varepsilon\rangle_{\omega_{1}} .
\end{aligned}
$$

Furthermore, in the Mean-Field algorithms it is assumed that the matrix and the inclusion phases follow the macroscopic stress-strain behavior in the microscale. This implies that the macroscopic stress-strain relation is valid for the average fields in the phases:

$$
\begin{aligned}
& \langle\boldsymbol{\sigma}\rangle_{\omega_{0}}=\mathcal{C}_{0}^{\mathrm{el}}:\langle\boldsymbol{\varepsilon}\rangle_{\omega_{0}}, \\
& \langle\boldsymbol{\sigma}\rangle_{\omega_{1}}=\mathcal{C}_{1}^{\mathrm{el}}:\langle\boldsymbol{\varepsilon}\rangle_{\omega_{1}}
\end{aligned}
$$

where $\mathcal{C}^{\mathrm{el}}$ is the macroscopic $4^{\text {th }}$ order elasticity tensor.

Equations (5.4) to (5.7) make up a system that has 6 variables and 4 equations. For the solution of the system, one of the variables is prescribed which still leaves one undetermined degree of freedom in the system. It is clear that to be able to completely determine all the variables in the system, there needs to be at least one more independent equation.

\subsubsection{Strain and Stress concentration}

The additional equation necessary to close the system is generally given in the form of a strain or stress concentration in the inhomogeneity. As the name suggests the equation relates the average strain or stress that is concentrated in a phase to the total average strain or stress. The strain concentration is expressed as,

$$
\langle\varepsilon\rangle_{\omega_{1}}=\mathcal{A}:\langle\varepsilon\rangle_{\omega}
$$


where $\mathcal{A}$ is the $4^{\text {th }}$ order strain concentration tensor. Using Equation (5.6), the strain localized in the matrix phase can be calculated as:

$$
\langle\varepsilon\rangle_{\omega_{0}}=\frac{1}{f_{0}}\left(\mathcal{I}-f_{1} \mathcal{A}\right):\langle\varepsilon\rangle_{\omega}
$$

where $\mathcal{I}$ is the $4^{\text {th }}$ order symmetric identity tensor:

$$
I_{i j k l}=\frac{1}{2}\left(\delta_{i k} \delta_{j l}+\delta_{i l} \delta_{j k}\right) .
$$

Another useful relation is the strain concentration between the two phases:

$$
\langle\varepsilon\rangle_{\omega_{1}}=\hat{\mathcal{A}}:\langle\varepsilon\rangle_{\omega_{0}}
$$

where, using Equations (5.8), (5.10) and (5.11)

$$
\hat{\mathcal{A}}=f_{0} \mathcal{A}:\left(\mathcal{I}-f_{1} \mathcal{A}\right)^{-1}, \quad \mathcal{A}=\hat{\mathcal{A}}:\left(f_{1} \hat{\mathcal{A}}+f_{0} \mathcal{I}\right)^{-1} .
$$

Following the same convention in terms of stress, the following equations are defined:

$$
\begin{aligned}
& \langle\boldsymbol{\sigma}\rangle_{\omega_{1}}=\mathcal{B}:\langle\boldsymbol{\sigma}\rangle_{\omega}, \\
& \langle\boldsymbol{\sigma}\rangle_{\omega_{1}}=\hat{\mathcal{B}}:\langle\boldsymbol{\sigma}\rangle_{\omega_{0}}
\end{aligned}
$$

where $\mathcal{B}$ is the $4^{\text {th }}$ order stress concentration tensor.

\subsubsection{Homogenized elastic response}

The equations defined above make it possible to get a homogenized elastic response of the RVE. An equivalent elastic operator can be defined that relates the average stress to the average strain in the RVE as,

$$
\langle\boldsymbol{\sigma}\rangle_{\omega}=\mathcal{C}^{\mathrm{el}}:\langle\varepsilon\rangle_{\omega}
$$

Combining Equations (5.4) and (5.7) gives,

$$
\langle\boldsymbol{\sigma}\rangle_{\omega}=f_{1} \mathcal{C}_{1}^{\mathrm{el}}:\langle\varepsilon\rangle_{\omega_{1}}+f_{0} \mathcal{C}_{0}^{\mathrm{el}}:\langle\varepsilon\rangle_{\omega_{0}},
$$

which, using Equation (5.8), becomes:

$$
\begin{aligned}
\langle\boldsymbol{\sigma}\rangle_{\omega} & =f_{1} \mathcal{C}_{1}^{\mathrm{el}}: \mathcal{A}:\langle\varepsilon\rangle_{\omega}+\mathcal{C}_{0}^{\mathrm{el}}:\left(\mathcal{I}-f_{1} \mathcal{A}\right):\langle\varepsilon\rangle_{\omega} \\
& =\left[\mathcal{C}_{0}^{\mathrm{el}}+f_{1}\left(\mathcal{C}_{1}^{\mathrm{el}}-\mathcal{C}_{0}^{\mathrm{el}}\right): \mathcal{A}\right]:\langle\varepsilon\rangle_{\omega} .
\end{aligned}
$$

Therefore, the homogenized elastic operator is obtained as:

$$
\mathcal{C}^{\mathrm{el}}=\mathcal{C}_{0}^{\mathrm{el}}+f_{1}\left(\mathcal{C}_{1}^{\mathrm{el}}-\mathcal{C}_{0}^{\mathrm{el}}\right): \mathcal{A} .
$$

In Equation (5.17), there are no assumptions on the strain concentration tensor making it a generally applicable equation for any $\mathcal{A}$. 


\subsubsection{Eshelby's solution}

Eshelby $[20,21]$ found an analytical solution to the strain concentration problem in the case where an inhomogeneity with different elastic properties is present in an infinitely large matrix, as illustrated in Figure 5.2.

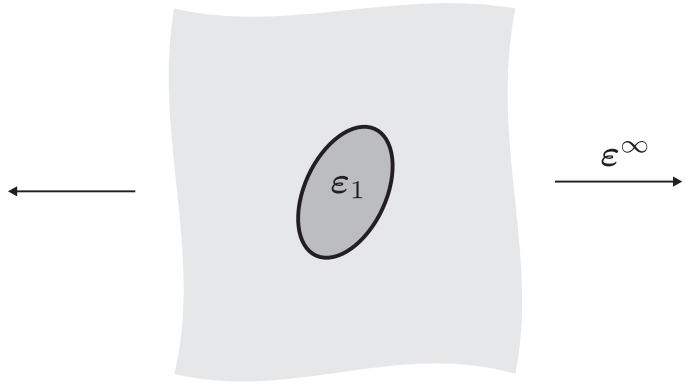

Figure 5.2: Eshelby's inhomogeneity problem; single inhomogeneity in an infinitely large matrix.

To find the solution, Eshelby first considered another fundamental problem which is to find the equilibrium state when an arbitrary region in the material (inclusion) undergoes a prescribed stress-free (transformation) strain. In other words, a region in a homogeneous material is deformed by a prescribed strain, which Eshelby refers to as the eigenstrain. This is considered a stress-free strain meaning that the material is not stressed to cause the shape change. Naturally, this eigenstrain disturbs the equilibrium with the surrounding matrix. Hence, the problem is to find the resulting strain inside the region after equilibrium is satisfied.

Eshelby solved this problem considering the process to occur in four stages which are schematically illustrated in Figure 5.3. First, the inclusion is cut out of the material and the eigenstrain, $\varepsilon^{*}$, is applied in an unconstrained setting. The resulting geometry is elastically deformed to regain the original shape of the inclusion by application of stress, i.e. $\boldsymbol{\sigma}^{*}=-\mathcal{C}^{\mathrm{el}}: \boldsymbol{\varepsilon}^{*}$. Since compatibility with the matrix is then satisfied, the cut-off section is patched back into its original location and the stress in the inclusion is allowed to relax. The resulting equilibrium strain in the cut-off region is given by:

$$
\tilde{\varepsilon}=\mathcal{E}\left(\mathcal{C}_{0}^{\mathrm{el}}\right): \varepsilon^{*}
$$

where $\mathcal{E}$ is the $4^{\text {th }}$ order Eshelby tensor and is a function of the stiffness of the matrix and the shape of the inclusion.

The solution of the inhomogeneity problem is related to the above problem via Eshelby's equivalent-inclusion theory. When a far-field strain is applied on a material with a single inhomogeneity, there will be strain and stress concentration due to the mismatch of the elastic properties of the matrix and the inhomogeneity, as shown in Figure 5.2. Eshelby's equivalent-inclusion theory states that the same stress field inside the inhomogeneity can be obtained by an inclusion undergoing a certain eigenstrain. 


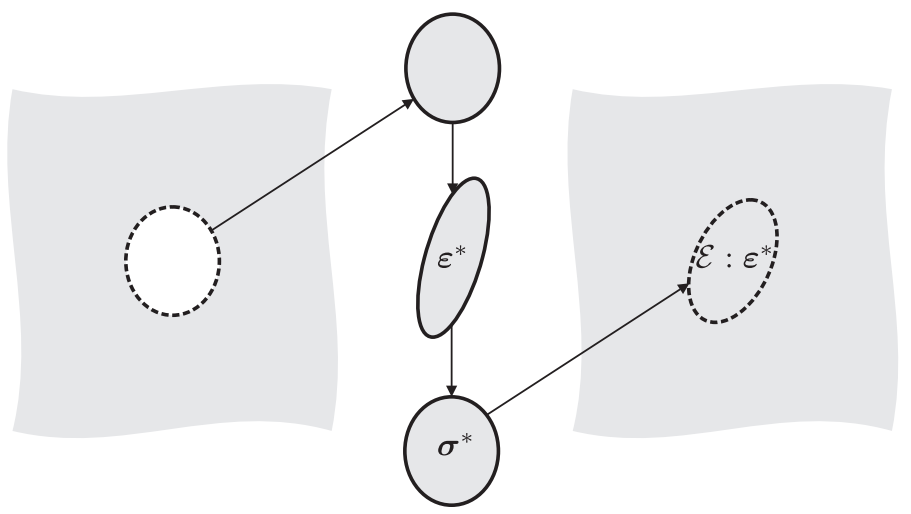

Figure 5.3: Eshelby's solution procedure of the inclusion problem.

The stress localized in the inhomogeneity is calculated as

$$
\begin{aligned}
\boldsymbol{\sigma}_{1} & =\mathcal{C}_{1}^{\mathrm{el}}: \varepsilon_{1} \\
& =\mathcal{C}_{1}^{\mathrm{el}}:\left(\varepsilon^{\infty}+\tilde{\varepsilon}\right)
\end{aligned}
$$

where $\varepsilon^{\infty}$ is the far-field strain and $\tilde{\varepsilon}$ is the disturbance strain inside the inhomogeneity.

As the equivalent inclusion theory states, this stress is equal to the stress in the matrix material in the same region which has experienced an eigenstrain, $\varepsilon^{*}$, and equilibrated:

$$
\sigma_{1}=\mathcal{C}_{0}^{\mathrm{el}}:\left(\varepsilon^{\infty}+\tilde{\varepsilon}-\varepsilon^{*}\right) .
$$

With some algebra, the eigenstrain term in the above equations is eliminated and a single equation that relates the strain in the inhomogeneity to the far-field strain is obtained:

$$
\varepsilon_{1}=\mathcal{H}: \varepsilon^{\infty}, \quad \mathcal{H}=\left[\mathcal{E}\left(\mathcal{C}_{0}^{\mathrm{el}}\right):\left(\mathcal{C}_{0}^{\mathrm{el}}{ }^{-1}: \mathcal{C}_{1}^{\mathrm{el}}-\mathcal{I}\right)+\mathcal{I}\right]^{-1}
$$

Equation (5.21) is the exact analytical solution to the problem when the matrix is infinitely large and there is a single isolated inclusion (see Figure 5.2). Eshelby also proved that if the inhomogeneity is ellipsoidal in shape the resulting localized strain field is uniform. He also derived the Eshelby tensor for ellipsoidal inclusions. Mura [61] derived analytical expressions of the Eshelby tensor for different geometries of the inclusions in isotropic materials. Gavazzi and Lagoudas [23] developed a numerical method to calculate Eshelby's tensor for arbitrarily shaped inclusions. The computation of the Eshelby tensor for spheroidal inclusions is described in Appendix A.

\subsubsection{Homogenization schemes}

Depending on the choice of the strain-concentration tensor in Equation (5.8), different homogenization schemes can be obtained [16]. Except for the Voigt and Reuss models, 

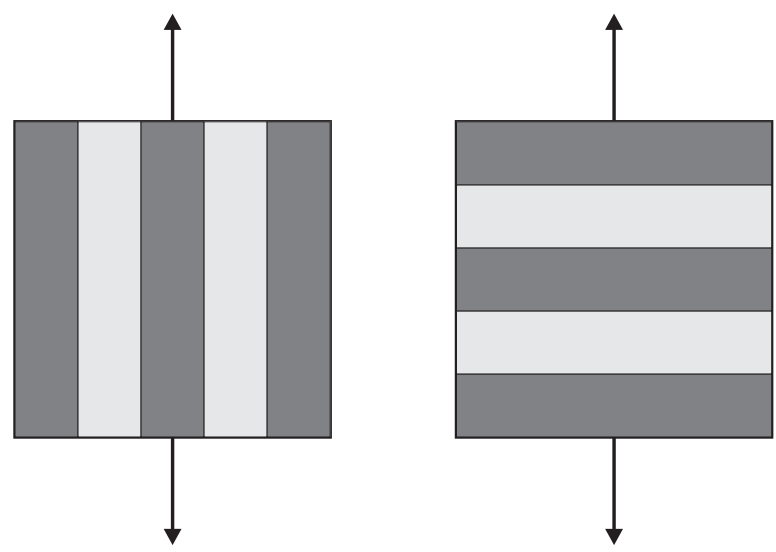

Figure 5.4: Idealized Voigt (left) and Reuss (right) schemes in an RVE.

most methods rely on Eshelby's analytical results which are exact for a single inclusion embedded in an infinitely long matrix. However, in reality a two-phase composite has a finite volume and many second-phase particles. In the Mean-Field methods, it is assumed that the behavior of all particles can be represented by a simplified matrix inclusion system. The homogenization schemes differ mostly in the way the far-field strain in Eshelby's solution is translated to the RVE.

\section{Voigt and Reuss models}

The simplest homogenization models were proposed by Voigt and Reuss. These models stem from the generalization as simple series and parallel connected laminates, as shown in Figure 5.4.

In the Voigt model a uniform strain field in the RVE is assumed, hence the strain in the matrix and the inclusions are the same. This results in a strain concentration tensor that is equal to the $4^{t h}$ order unit tensor:

$$
\begin{gathered}
\langle\varepsilon\rangle_{\omega_{0}}=\langle\varepsilon\rangle_{\omega_{1}}=\langle\varepsilon\rangle_{\omega}, \\
\mathcal{A}=\hat{\mathcal{A}}=\mathcal{I} .
\end{gathered}
$$

One of the results of the Voigt model is that the elastic operator of the composite is equal to the mixture rule applied on the elastic operator of the phases:

$$
\mathcal{C}^{\mathrm{el}}=f_{1} \mathcal{C}_{1}^{\mathrm{el}}+f_{0} \mathcal{C}_{0}^{\mathrm{el}}
$$

The Voigt model serves as an upper bound for composite materials meaning that the stiffness of the composite cannot be higher than the mixture of the two.

In the Reuss model, on the other hand, a uniform stress field in the RVE is assumed and hence equal stress distribution is obtained between the phases. Consequently, the 
stress concentration tensor is equal to unity and the strain concentration tensors are calculated as:

$$
\begin{gathered}
\mathcal{B}=\mathcal{I} \\
\hat{\mathcal{A}}=\mathcal{C}_{1}^{\mathrm{el}^{-1}}: \mathcal{C}_{0}^{\mathrm{el}}, \\
\Rightarrow \mathcal{A}=\left[f_{1} \mathcal{I}+f_{0} \mathcal{C}_{0}^{\mathrm{el}^{-1}}: \mathcal{C}_{1}^{\mathrm{el}}\right]^{-1}
\end{gathered}
$$

The mixture rule also applies in the homogenized response of the composite but on the compliance instead of the stiffness:

$$
\mathcal{C}^{\mathrm{el}-1}=f_{1} \mathcal{C}_{1}^{\mathrm{el}-1}+f_{0} \mathcal{C}_{0}^{\mathrm{e}^{-1}}
$$

The Reuss model serves as a lower-bound to the problem meaning, the compliance of the mixture cannot be lower than that predicted by the mixture rule.

\section{Mori-Tanaka model}

In the Mori-Tanaka model, as opposed to Voigt and Reuss models, the interaction between the particle and the matrix is taken into account [19, 83, 84]. This is accomplished by considering Eshelby's solution inside the RVE.

The derivation starts by considering that the average strain in the matrix is different from the far-field strain by a disturbance strain, $\varepsilon^{\prime}$, due to the presence of inhomogeneities. It is not possible to determine the disturbance strain field but in the Mori-Tanaka approach it is sufficient to consider the average disturbance strain. This makes the average strain in the inclusions and the matrix,

$$
\begin{aligned}
& \langle\varepsilon\rangle_{\omega_{0}}=\varepsilon^{\infty}+\left\langle\varepsilon^{\prime}\right\rangle, \\
& \langle\varepsilon\rangle_{\omega_{1}}=\varepsilon^{\infty}+\mathcal{E}\left(\mathcal{C}_{0}^{\mathrm{el}}\right): \varepsilon^{*}+\left\langle\varepsilon^{\prime}\right\rangle
\end{aligned}
$$

where $\varepsilon^{*}$ is the eigenstrain in the inclusion theory.

The equivalent inclusion theory allows that

$$
\mathcal{C}_{0}^{\mathrm{el}}:\left(\varepsilon^{\infty}+\mathcal{E}\left(\mathcal{C}_{0}^{\mathrm{el}}\right): \varepsilon^{*}+\left\langle\varepsilon^{\prime}\right\rangle-\varepsilon^{*}\right)=\mathcal{C}_{1}^{\mathrm{el}}:\left(\varepsilon^{\infty}+\mathcal{E}\left(\mathcal{C}_{0}^{\mathrm{el}}\right): \varepsilon^{*}+\left\langle\varepsilon^{\prime}\right\rangle\right)
$$

Eliminating $\varepsilon^{*}$ in Equation (5.30), the strain concentration tensor is obtained:

$$
\langle\varepsilon\rangle_{\omega_{1}}=\left[\mathcal{E}\left(\mathcal{C}_{0}^{\mathrm{el}}\right):\left(\mathcal{C}_{0}^{\mathrm{el}}{ }^{-1}: \mathcal{C}_{1}^{\mathrm{el}}-\mathcal{I}\right)+\mathcal{I}\right]^{-1}:\langle\varepsilon\rangle_{\omega_{0}}
$$

Equation (5.31) reveals that the strain concentration tensor, $\hat{\mathcal{A}}$ (see Equation (5.11)), is exactly the one that has been derived by Eshelby. Consequently, it is clear that in the Mori-Tanaka scheme it is assumed that each inclusion sees the matrix strain as the far-field strain [3]. This is an acceptable assumption for low volume fraction of inhomogeneities since in that case, the particles will be apart from each other, experiencing the matrix as the far-field. This leads to a deduction that this scheme is not suited for composites with high volume fraction of inclusions. It is also worth mentioning that if the properties of the matrix and the inclusion are exchanged and the volume fractions are switched (reverse Mori-Tanaka model), the results of the model will change, although intuitively one expects the same results. 


\section{Self-Consistent model}

In the Self-Consistent model [34, 46], the inclusion is assumed to be embedded in a matrix with the homogenized elastic properties of the RVE. The homogenized properties are of course yet to be determined, which makes the Self-Consistent model an implicit scheme.

Since the far-field strain is the average strain on the RVE,

$$
\langle\varepsilon\rangle_{\omega}=\varepsilon^{\infty},
$$

the average strain in the inclusions becomes,

$$
\langle\varepsilon\rangle_{\omega_{1}}=\langle\varepsilon\rangle_{\omega}+\mathcal{E}\left(\mathcal{C}^{\mathrm{el}}\right): \varepsilon^{*}
$$

where the Eshelby tensor has been computed by the homogenized elastic properties of the RVE, $\mathcal{C}$, given by Equation (5.17).

Applying the equivalent inclusion theory gives:

$$
\mathcal{C}_{1}^{\mathrm{el}}:\left(\langle\varepsilon\rangle_{\omega}+\mathcal{E}\left(\mathcal{C}^{\mathrm{el}}\right): \varepsilon^{*}\right)=\mathcal{C}^{\mathrm{el}}:\left(\langle\varepsilon\rangle_{\omega}+\mathcal{E}\left(\mathcal{C}^{\mathrm{el}}\right): \varepsilon^{*}-\varepsilon^{*}\right)
$$

and by eliminating $\varepsilon^{*}$ from the equations, the strain concentration tensor is obtained as

$$
\langle\varepsilon\rangle_{\omega_{1}}=\left[\mathcal{E}\left(\mathcal{C}^{\mathrm{el}}\right):\left(\mathcal{C}^{\mathrm{el}^{-1}}: \mathcal{C}_{1}^{\mathrm{el}}-\mathcal{I}\right)+\mathcal{I}\right]^{-1}:\langle\varepsilon\rangle_{\omega}
$$

Looking at Equation (5.35) in the perspective of Eshelby's solution, it is clear that the far-field strain that the inclusions experience is replaced with the average strain in the RVE, which is a better approximation than the Mori-Tanaka model. This property ensures that the model will preserve the validity even for high volume fraction of inclusions.

One other positive property of the Self-Consistent model is that when the matrix and the inclusion material properties are interchanged and the volume fractions are swapped, the solution remains the same.

The negative side however, is that the homogenized elastic operator in Equation (5.35) is one of the unknowns in the system, which makes the equations non-linear, requiring an iterative solution method. In the current study, a Fixed-Point iteration method is used for the solution of equations.

\section{Lielens interpolative model}

It was mentioned that in the Mori-Tanaka model the inclusions experience the matrix strain as the far-field strain and this assumption works well when the volume fraction of the inclusions is low but fails when it is high. When the material parameters and the volume fractions are swapped the reverse Mori-Tanaka model is obtained. In this form, the model is again valid for low volume fractions. Additionally, Hashin and Shtrikman [29] developed bounds using a variational formulation and these bounds correspond 
to the forward and reverse Mori-Tanaka results in case of two-phase composites with isotropic moduli.

Built on this observation, Lielens proposed an interpolative method to determine the strain concentration tensor, using forward and reverse Mori-Tanaka approximations as a function of the volume fractions [17, 18]:

$$
\begin{aligned}
\hat{\mathcal{A}}_{\mathrm{fw}} & =\left[\mathcal{E}\left(\mathcal{C}_{0}^{\mathrm{el}}\right):\left(\mathcal{C}_{0}^{\mathrm{el}}{ }^{-1}: \mathcal{C}_{1}^{\mathrm{el}}-\mathcal{I}\right)+\mathcal{I}\right]^{-1}, \\
\hat{\mathcal{A}}_{\mathrm{rv}} & =\left[\mathcal{E}\left(\mathcal{C}_{1}^{\mathrm{el}}\right):\left(\mathcal{C}_{1}^{\mathrm{el}}{ }^{-1}: \mathcal{C}_{0}^{\mathrm{el}}-\mathcal{I}\right)+\mathcal{I}\right]^{-1}, \\
\hat{\mathcal{A}} & =\left[\phi\left(f_{1}\right) \hat{\mathcal{A}}_{\mathrm{rv}}^{-1}+\left(1-\phi\left(f_{1}\right)\right) \hat{\mathcal{A}}_{\mathrm{fw}}^{-1}\right]^{-1}
\end{aligned}
$$

where $\phi$ is the interpolation function.

In the limiting cases, Lielens proposed that the model should yield the forward and reverse Mori-Tanaka approximations; lower and upper bounds, respectively. Hence, the following conditions must hold for the chosen interpolation function:

$$
0 \leq \phi\left(f_{1}\right) \leq 1, \quad \lim _{f_{1} \rightarrow 0} \phi\left(f_{1}\right)=0, \quad \lim _{f_{1} \rightarrow 1} \phi\left(f_{1}\right)=1
$$

The Lielens model can be put in a physical perspective by considering the effect of the volume fraction of inclusions on the microstructure. For low volume fractions, the inclusions are far apart from each other and hence the forward Mori-Tanaka approximation is valid. Increasing the volume fraction, the particles start to approach each other and beyond some point they start to interconnect and become the matrix. After this point the original matrix phase starts to appear in particles thus becoming the inclusions. For higher volume fractions there will be only a number of matrix particles left in the microstructure.

Consequently, by choosing a suitable interpolation function different types of behavior can be captured. The original function that Lielens proposed is,

$$
\phi^{\mathrm{Li}}\left(f_{1}\right)=\frac{f_{1}+f_{1}^{2}}{2} .
$$

It is possible to use other interpolation functions fulfilling the conditions in Equation (5.37) to get a better approximation of the physics. One method is for example to chose the function so that the results of the Self-Consistent model are resembled. The forward and reverse Mori-Tanaka models are satisfactory in the vicinity of the limits however, the Self-Consistent method is thought to give better approximation for moderate volume fractions.

\section{Bound-Interpolation model}

Motivated by the Lielens model, a simpler interpolative approximation can be performed. Lielens proposed using the forward and reverse Mori-Tanaka approximations as the bounds and interpolating between them with the condition 
that at the lower and upper limits they have to be satisfied, respectively. An easier approach would be to interpolate between the more relaxed bounds, Voigt and Reuss. In this approach, since it is purely phenomenological, there is no need for the evaluation of the Eshelby tensor. The strain concentration tensor then becomes,

$$
\hat{\mathcal{A}}=\left[\phi\left(f_{1}\right) \mathcal{I}+\left(1-\phi\left(f_{1}\right)\right) \mathcal{C}_{0}^{\mathrm{el}}{ }^{-1}: \mathcal{C}_{1}^{\mathrm{el}}\right]^{-1}
$$

The interpolation function should satisfy,

$$
0 \leq \phi\left(f_{1}\right) \leq 1, \quad \lim _{f_{1} \rightarrow 0} \phi\left(f_{1}\right) \geq 0, \quad \lim _{f_{1} \rightarrow 1} \phi\left(f_{1}\right) \leq 1 .
$$

It must be kept in mind that this approach has no direct physical basis. On the other hand, it gives better approximations than the Voigt and Reuss models.

\subsubsection{Comparison of models}

To compare different models, a mixture of two isotropic elastic materials was considered where, the matrix has lower elastic modulus compared to the inclusions. Two cases were considered: first with a small contrast in the elastic properties and second with a high contrast. The results are presented in terms of the homogenized elastic moduli of the composite versus the volume fraction of the inclusions.

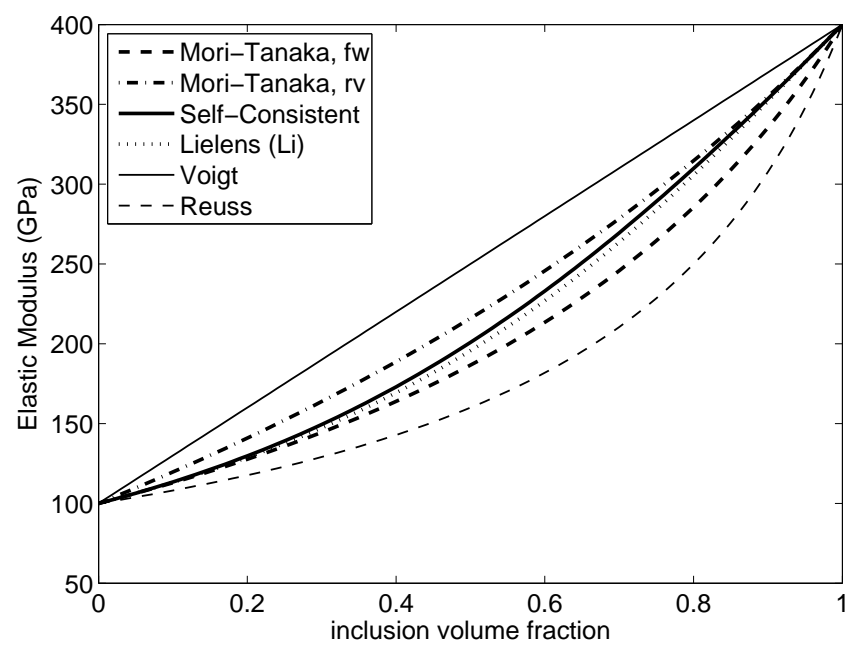

Figure 5.5: Homogenized elastic modulus vs. volume fraction of the inclusions. $E_{0}=100 \mathrm{GPa}, E_{1}=400 \mathrm{GPa}, \nu_{0}=\nu_{1}=0.33$.

Figure 5.5 shows the calculated elastic modulus of the composite against the volume fraction of the inclusions which are assumed spherical in shape. Since the contrast 
between the elastic properties of the phases is not very large, almost all models (except for the bounds) give acceptable results.

The Self-Consistent approach is expected to give the most reliable results considering the underlying assumptions in the models. It is observed that the Mori-Tanaka results follow the Self-Consistent results up to around $30 \%$ of inclusions. The same holds for the reverse Mori-Tanaka model in the reverse direction. It can be seen however that outside these limits, the Mori-Tanaka results depart from the Self-Consistent results. It is observed that Lielens interpolation works very well in this case as it interpolates between the forward and reverse Mori-Tanaka approximation.

It is worth mentioning again that the Mori-Tanaka model is based on the assumption that the inclusions are separated, as opposed to the Self-Consistent model which does not have that restriction due to its implicit nature. It is seen on Figure 5.5 that the approximation holds quite well for low volume fractions. Consequently, the Self-Consistent model is always expected to give better results than the Mori-Tanaka model. Since the Lielens interpolation is based on the Mori-Tanaka models, it is expected to converge to the Self-Consistent model in the best case scenario. As a conclusion, it is not correct to claim that any of the models presented above is better than the Self-Consistent model, since theoretically it is the most reliable model.

When the elastic contrast between the phases is very significant (e.g. a polymer matrix composite), the assumptions made in the modeling become very important.

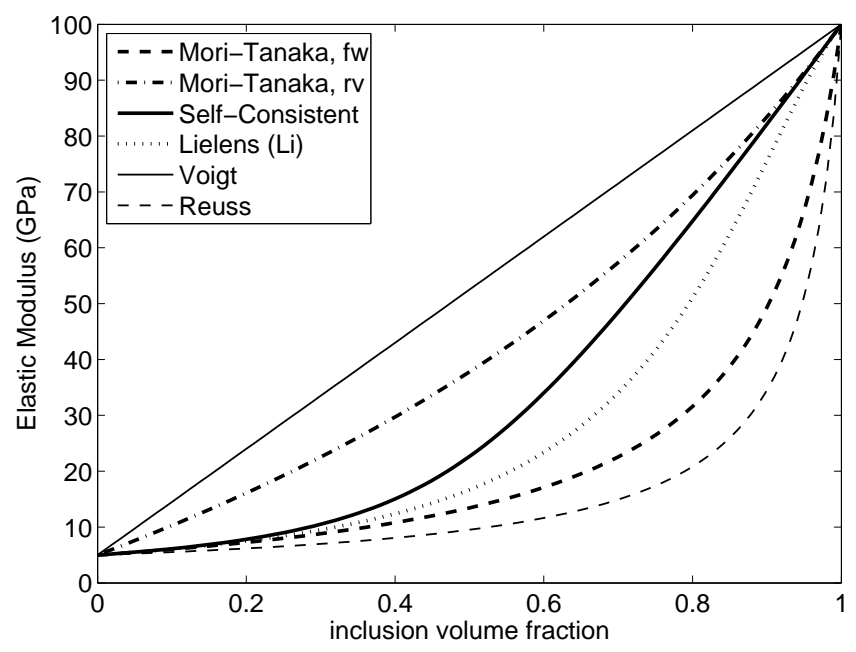

Figure 5.6: Homogenized elastic modulus vs. volume fraction of the inclusions. $E_{0}=5 \mathrm{GPa}, E_{1}=100 \mathrm{GPa}, \nu_{0}=\nu_{1}=0.33$.

It is shown in Figure 5.6 that when the elastic contrast is larger, the different model results separate from each other. Still, the Mori-Tanaka approximation holds quite well for low volume fractions but underestimates the stiffness significantly at higher 
volume fractions. Lielens model, although much better than the Mori-Tanaka model, also underestimates the stiffness compared to the Self-Consistent model.

However, the Lielens interpolation function can be replaced with a stronger approximation of the Self-Consistent using a sigmoid function:

$$
\phi^{\mathrm{PG}}\left(f_{1}\right)=\frac{d_{1}}{1+\exp \left(\left(c_{1}-f_{1}\right) c_{2}\right)}-d_{2} .
$$

where $c_{1}$ defines the volume fraction at which the forward and reverse Mori-Tanaka approximations are switched (the switching point of the sigmoid) and $c_{2}$ defines the sharpness of the switch, respectively. The dependent parameters, $d_{1}$ and $d_{2}$, ensure that the conditions (5.37) are satisfied. It is possible to make the $c$ parameters functions of the elastic contrast for better accuracy. The results of this interpolation are shown in Figure 5.7 for the same problem as in Figure 5.6. The same interpolation function, with different parameters, can be used for the Bound-Interpolation model and a good fit to the Self-Consistent results can be obtained. The advantages of the Bound-Interpolation model is that it is explicit and it does not require any computation of the Eshelby tensor.

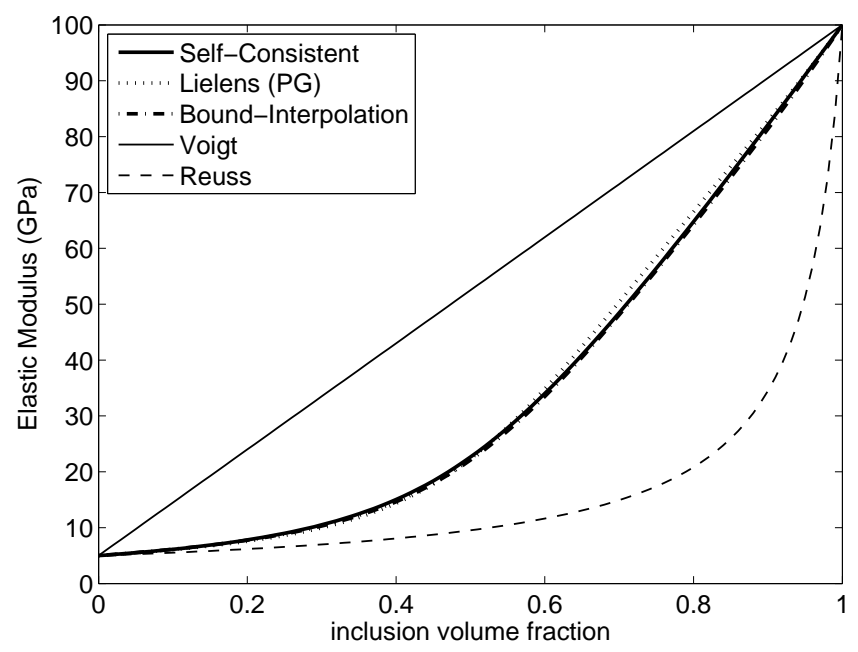

Figure 5.7: Homogenized elastic modulus vs. volume fraction of the inclusions. $E_{0}=5 \mathrm{GPa}, E_{1}=100 \mathrm{GPa}, \nu_{0}=\nu_{1}=0.33$.

\subsection{Homogenization of elastic-plastic composites}

In the previous section the Mean-Field homogenization method for linear elastic composite materials was introduced. This section focuses on the application of the same method to elastic-plastic composites. 
As discussed in the previous section, most of the Mean-Field homogenization schemes depend on Eshelby's solution of the inhomogeneity problem which gives the exact solution for an elastic inhomogeneity embedded in an infinitely large elastic matrix. Iwakuma and Nemat-Nasser [39, 64] extended Eshelby's theory for large deformations and elastic-plastic materials.

There are several complications of utilizing the Eshelby solution in elastic-plastic homogenization problems. First of all, evidently, the material is not elastic anymore. Therefore, the material behavior must be linearized to an equivalent reference material to which the Eshelby method can be applied. There are a number of different possible approaches to the linearization problem that will be discussed below.

Another problem is the validity of the small strain assumption. For elastic solutions on metals, the small strain assumption is generally applicable since the attainable elastic strains are very small. However, when large deformations are involved the small strain assumption does not hold. Therefore, a more general strain definition needs to be used in the homogenization model.

\subsubsection{Large deformations}

In metal plasticity, the elastic-plastic deformation is commonly modeled using a hypoelastic-plastic formulation. In this formulation, linear elasticity is assumed (as opposed to the hyperelastic-plastic formulation) and furthermore energy is not conserved in a closed deformation cycle [2]. On the other hand, this formulation holds quite well for metals due to the fact that the elastic strains are very small so that the non-linear regime in elasticity is not reached and the energy error is insignificant [2]. The constitutive models for large deformations are summarized and discussed in Chapter 6.

For materials with elastic isotropy, the relation between the Jaumann rate of Cauchy stress and the deformation rate is given $\operatorname{as}^{1}$ :

$$
\stackrel{\nabla}{\boldsymbol{\sigma}}=\mathcal{C}^{\mathrm{J}, \mathrm{el}}: \mathbf{D}^{e}=\mathcal{C}^{\mathrm{J}, \mathrm{el}}:\left(\mathbf{D}-\mathbf{D}^{\mathrm{p}}\right)=\mathcal{C}^{\mathrm{J}}: \mathbf{D}
$$

(for details of the formulation see Section 6.3.1).

Consequently when plasticity is involved, the elastic stress-strain relation in the homogenization algorithms has to be replaced by the elastic-plastic one.

For homogenization equations to hold, the averaging conditions must be satisfied for large deformations [63]. The averaging equation given in Section 5.1.1 can be applied to the deformation gradient in the following manner:

$$
\langle\mathbf{F}\rangle_{\Omega}=\frac{1}{V_{\Omega}} \int_{\Omega} \frac{\partial \mathbf{x}}{\partial \mathbf{X}} \mathrm{d} V
$$

\footnotetext{
${ }^{1}$ For elastically anisotropic materials, care must be taken using the elastic operator with objective rates $[2]$.
} 
where $\Omega$ represents the volume in the reference configuration. The velocity gradient is averaged as:

$$
\langle\mathbf{L}\rangle_{\omega}=\frac{1}{V_{\omega}} \int_{\omega} \frac{\partial \mathbf{v}}{\partial \mathbf{X}} \cdot \frac{\partial \mathbf{X}}{\partial \mathbf{x}} \mathrm{d} V=\left\langle\dot{\mathbf{F}} \cdot \mathbf{F}^{-1}\right\rangle_{\omega}
$$

where $\omega$ represents the volume in the current configuration.

The macroscopic velocity gradient, if calculated using the macroscopic deformation gradient, is calculated as:

$$
\overline{\mathbf{L}}=\dot{\overline{\mathbf{F}}} \cdot \overline{\mathbf{F}}^{-1}=\langle\dot{\mathbf{F}}\rangle_{\Omega} \cdot\langle\mathbf{F}\rangle_{\Omega}{ }^{-1}
$$

To be able to use the average deformation rate in the homogenization algorithm, Equations (5.44) and (5.45) must be equal. This condition is only satisfied when the current configuration coincides with the reference configuration [63], i.e. $\mathbf{F}=\mathbf{I}$, which usually does not hold. However, when small increments are considered the averaging error reduces:

$$
\lim _{\delta \mathbf{u} \rightarrow 0} \mathbf{F}=\mathbf{I}
$$

hence, for small $\delta u$ :

$$
\overline{\mathbf{L}} \approx\langle\mathbf{L}\rangle_{\omega}
$$

Nemat-Nasser and Iwakuma [39, 64] showed that in case of large deformations, Eshelby's theory is still valid and the Eshelby tensor is the same as in the linear theory.

\subsubsection{Linearization of elastic-plastic response}

Eshelby's solution is based on the inclusion theory which allows finding the equilibrium strain field inside the inclusion when it undergoes an eigenstrain. The solution is obtained by considering the stresses that are relaxed after patching the inclusion back. Therefore, the Eshelby tensor, as well as the whole theory, depends on the stress-strain relationship of the matrix and the inclusion. In the elastic regime the procedure is very straightforward. On the other hand, in the elastic-plastic regime it is more complicated to find the exact stress-state for a given strain increment.

Hill [33] used an incremental formulation in the Self-Consistent homogenization algorithm where the elastic operator is replaced with the elastoplastic operator, i.e. continuum tangent:

$$
\stackrel{\nabla}{\boldsymbol{\sigma}}=\mathcal{C}^{\mathrm{J}}: \mathbf{D}
$$

This approach is followed by others for different homogenization algorithms [18]. Equation (5.48) gives a physical relation between the strain rate and the stress rate. A general form of $\mathcal{C}^{\mathrm{J}}$ in the hypoelastic-plastic formulation is given by (see Section 6.3.1):

$$
\mathcal{C}^{\mathrm{J}}=\mathcal{C}^{\mathrm{J}, \mathrm{el}}-\frac{\left(\mathcal{C}^{\mathrm{J}, \mathrm{el}}: \mathbf{r}\right) \otimes\left(\frac{\partial \phi}{\partial \boldsymbol{\sigma}}: \mathcal{C}^{\mathrm{J}, \mathrm{el}}\right)}{-\frac{\partial \phi}{\partial \mathbf{q}} \cdot \mathbf{h}+\frac{\partial \phi}{\partial \mathbf{q}}: \mathcal{C}^{\mathrm{J}, \mathrm{el}}: \mathbf{r}}
$$


where $\phi$ is the yield function, $\mathbf{r}$ is the plastic flow direction, $\mathbf{q}$ represents the vector of hardening variables and $\mathbf{h}$ is the hardening function.

Another approach would be using the algorithmic tangent modulus [78] which relates the increment of stress to increment of strain. For vanishingly small increments it is equal to the continuum tangent modulus. This operator is generally used in finite element calculations because it results in a consistent stiffness matrix. It has been used by Doghri and Ouaar [18] successfully in Mori-Tanaka and Lielens models. However, this operator is dependent on the size of the prescribed displacement and the update algorithm used, which raises concerns about the physical validity of this approach.

Another form of linearization is the secant modulus formulation which has been used in several studies for Self-Consistent [4] and Mori-Tanaka [84] approaches. In this method, the total stress is related to the total accumulated strain via the secant modulus:

$$
\boldsymbol{\sigma}=\mathcal{C}^{\mathrm{sec}}: \varepsilon \text {. }
$$

Although it is difficult to put this method into a physical perspective considering Eshelby's solution, it is demonstrated by some authors that using secant modulus in the homogenization yields better results than the direct application of the incremental formulation [25]. On the other hand, it is obvious that this form of linearization is only reliable when the deformation path is monotonic and proportional. Moreover, determination of the secant modulus requires solution of a set of non-linear equations.

\subsubsection{Isotropic projection of the tangent moduli}

It has been observed that using the continuum tangent operators directly in the homogenization scheme gives results that are too stiff [25]. Pierard [73] and Doghri [18] compared different implementations of the algorithm by using an isotropic projection for selected tangent operators. Their results showed that by calculating only the Eshelby tensor with the projected tangent operator, recovers the consistency of the method and gives good results. It can be deducted from this point of view that the problem resides in generalization of Eshelby's inclusion theory to elastic-plastic materials.

Isotropic projection can be described as distributing the directional properties of a tensor to every direction. In plasticity, generally, the material is softer in the direction of yielding but harder in other directions. Any isotropic $4^{\text {th }}$ order tensor $\mathcal{D}^{\text {iso }}$ can be written as [55]:

$$
\begin{gathered}
\mathcal{D}^{\text {iso }}=\alpha \mathcal{I}^{\mathrm{vol}}+\beta \mathcal{I}^{\mathrm{dev}} \\
\alpha=\mathcal{I}^{\mathrm{vol}}:: \mathcal{D}^{\text {iso }}, \quad \beta=\frac{1}{5} \mathcal{I}^{\mathrm{dev}}:: \mathcal{D}^{\text {iso }}
\end{gathered}
$$

where $\mathcal{I}^{\text {vol }}$ and $\mathcal{I}^{\text {dev }}$ are the volumetric (spherical) and deviatoric parts of the $4^{\text {th }}$ order identity tensor (see Equation (5.10)):

$$
\mathcal{I}^{\mathrm{vol}}=\frac{1}{3} \mathbf{I} \otimes \mathbf{I}, \quad \mathcal{I}^{\mathrm{dev}}=\mathcal{I}-\mathcal{I}^{\mathrm{vol}}
$$


There is no unique definition of the isotropic projection. However, considering the above property of the isotropic tensors, it is possible to define a general projection operation:

$$
\begin{gathered}
\mathcal{D}^{\text {iso }}=\alpha \mathcal{I}^{\mathrm{vol}}+\beta \mathcal{I}^{\mathrm{dev}} \\
\alpha=\mathcal{I}^{\mathrm{vol}}:: \mathcal{D}^{\text {aniso }}, \quad \beta=\frac{1}{5} \mathcal{I}^{\mathrm{dev}}:: \mathcal{D}^{\text {aniso }} .
\end{gathered}
$$

Pierard's results also demonstrate that using the projected tangent operators for all the moduli in the algorithm results in too stiff (close to Voigt) results [73].

\subsubsection{Solution algorithm}

The purpose of the homogenization algorithm is to find the converged solution for all the state variables in both phases as well as the averaged response. To achieve this, the following set of nonlinear equations must be solved for a given macroscopic strain increment, $\overline{\mathbf{D}}$ :

$$
\begin{aligned}
& \overline{\mathbf{D}}=\langle\mathbf{D}\rangle_{\omega} \\
& \langle\stackrel{\nabla}{\boldsymbol{\sigma}}\rangle_{\omega_{0}}=\mathcal{C}_{0}^{\mathrm{J}}:\langle\mathbf{D}\rangle_{\omega_{0}} \\
& \langle\stackrel{\nabla}{\boldsymbol{\sigma}}\rangle_{\omega_{1}}=\mathcal{C}_{1}^{\mathrm{J}}:\langle\mathbf{D}\rangle_{\omega_{1}} \\
& \langle\mathbf{D}\rangle_{\omega_{1}}=\mathcal{A}\left(\mathcal{C}_{0}^{\mathrm{J}}, \mathcal{C}_{1}^{\mathrm{J}}, \mathcal{E}\right):\langle\mathbf{D}\rangle_{\omega} \\
& \langle\mathbf{D}\rangle_{\omega}=f_{1}\langle\mathbf{D}\rangle_{\omega_{1}}+f_{0}\langle\mathbf{D}\rangle_{\omega_{0}} \text {. }
\end{aligned}
$$

Doghri $[17,18]$ showed that the above equations can be solved using the Fixed-Point iteration method which is robust and fast in converging to a solution. The iterations start with taking $\mathcal{A}=\mathcal{I}$ (Voigt assumption) and partitioning the strains accordingly. The stress-update algorithms of each phase are called and the continuum elasticplastic tangents are calculated. These are then used to update the strain concentration tensor for the next iteration. The iterations continue until the change in $\langle\mathbf{D}\rangle_{\omega_{1}}$ in two successive steps is less than the imposed tolerance.

\subsubsection{Results and discussion}

The results of the algorithms discussed above are presented on two different example problems. The first problem is the elastic-plastic simulation of a well known metalmatrix composite material. On this example, the results of the models are compared with a Direct Finite Element computation. The next problem is related to the austenitic stainless steels. A composite material is considered with austenite and martensite phases as the constituents. Results of the different algorithms are discussed and put in perspective. 


\section{Metal-Matrix Composite}

For validation purposes, a well-known problem was selected which was studied previously by other researchers for different homogenization algorithms. Hence, it is possible to find Direct Finite Element method results for this material which are used for verification of the Mean-Field method that has been implemented. It is difficult to find experimental data on elastic-plastic composites to have a solid validation of the models and commonly the Direct Finite Element method is utilized for comparison purposes.

The material consists of an elastic-plastic matrix (aluminum alloy) and spherical elastic (ceramic) inclusions. The elastic properties of the phases are also different. The volume fraction of the inclusions is 0.2 . The matrix is assumed to follow J2-flow plasticity with an isotropic power-law strain hardening in the form $\sigma^{\mathrm{f}}=K\left(\varepsilon^{\mathrm{p}}\right)^{m}$. The material parameters of the two phases are summarized in Table 5.1.

Table 5.1: Material parameters used for the matrix and inclusion phases.

\begin{tabular}{rrcccc}
\hline Phase & $E(\mathrm{MPa})$ & $\nu$ & $\sigma^{\mathrm{y}}(\mathrm{MPa})$ & $K$ & $m$ \\
\hline Matrix & 75000 & 0.3 & 75 & 416 & 0.3895 \\
Inclusions & 400000 & 0.2 & - & - & - \\
\hline
\end{tabular}

In Figure 5.8, the results of the implemented Self-Consistent and Lielens models are compared to Direct Finite Element calculations performed by Doghri and Ouaar [18]. A very good correspondence is obtained by both models in terms of the macroscopic response of the composite which supports the validity of the implemented Mean-Field algorithms.

\section{Austenite-Martensite}

The comparison of different algorithms with each other is performed on another example problem which is more closely related to the metastable austenitic stainless steels. The composite material, in this case, is comprised of spherical martensite inclusions embedded in an austenite matrix. It must be mentioned that it is very difficult to determine the mechanical properties of the individual phases in such a composite material. Therefore, the general procedure in homogenization approaches is to use the macroscopic material data in microscale and assume that the material shows the same behavior when it is a part of the composite. The macroscopic data are best obtained by producing a single-phase material under similar conditions to the actual multi-phase material and do mechanical testing on the phases separately. However, this in itself might be a challenging task since some phases cannot be produced separately.

In case of metastable austenitic stainless steels, performing tests on austenite is easier than on martensite. The critical point is to avoid transformation during the deformation of austenite. This can be achieved by doing the tests at elevated temperature and extrapolating the results to room temperature. 


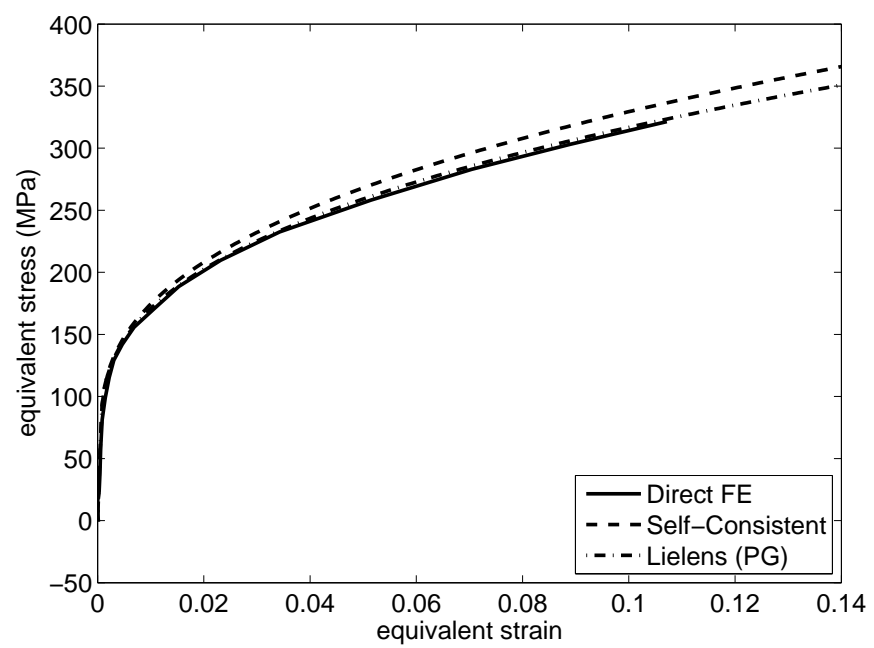

Figure 5.8: Comparison of the Self-Consistent and Lielens models with Direct Finite Element method results [18] for an aluminum alloy with spherical ceramic inclusions.

Determining the mechanical response of martensite is more difficult. During the mechanically induced transformation, the martensite particles carry load and some of them yield plastically. Therefore, after a test that results in a complete transformation, it is not possible to test the specimen again and gather reliable data. Additionally, there might be a difference in mechanical behavior of martensite when it is transformed athermally or isothermally. Therefore, it is also not perfectly reliable to transform the material first with another method and perform a test on the martensite phase.

Post [74] relied on this second method and transformed the material isothermally under stress. Then, by performing upsetting tests determined the mechanical behavior of austenite and martensite. It can be observed that the martensite measured by his experiments is remarkably softer than martensite found in other steels, due to the low carbon content of the alloy. The austenite and martensite parameters that are used below are based on Post's results. Austenite parameters are slightly modified to reproduce the experimentally determined yield stress.

For both materials, the phenomenological Ludwig-Nadai strain hardening model is used which has the form:

$$
\sigma^{\mathrm{f}}=\sigma^{0}+K\left(\varepsilon^{0}+\varepsilon^{\mathrm{p}}\right)^{m}
$$

where $\sigma^{\mathrm{f}}$ is the flow stress and $\varepsilon^{\mathrm{p}}$ is the equivalent plastic strain. The parameters used for austenite and martensite are summarized in table 5.2. Additionally, for both materials, J2 plasticity is assumed.

Figure 5.9 shows the equivalent stress vs. equivalent strain curves obtained with parameters given in Table 5.2. 
Table 5.2: Material parameters used for the austenite and martensite phases.

\begin{tabular}{rrccccc}
\hline Phase & $E(\mathrm{MPa})$ & $\nu$ & $\sigma^{\mathrm{y}}(\mathrm{MPa})$ & $\varepsilon^{0}$ & $K$ & $m$ \\
\hline Austenite & 210000 & 0.3 & 280 & 0.01 & 1000 & 0.51 \\
Martensite & 210000 & 0.3 & 800 & 0.001 & 1000 & 0.07 \\
\hline
\end{tabular}

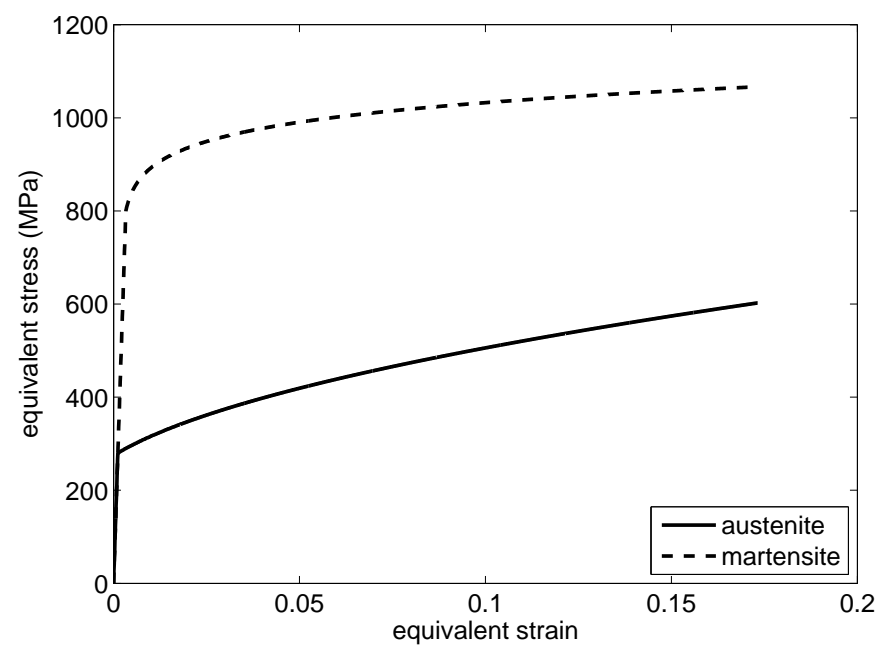

Figure 5.9: Stress-strain behavior of the martensite and austenite phases.

As mentioned, the martensite particles are assumed to be spherical. Although this might not represent the actual shape of a single inclusion, for many inclusions with no preferential direction, it can be considered a reasonable assumption. This can be justified by considering a large number of particles, all ellipsoidal but randomly oriented, in the austenite matrix. The composite in this situation is expected to have isotropic average properties since there is no preferential direction. If, on the other hand, an ellipsoid inclusion would have been used, the resulting properties would not be isotropic. Furthermore, continuum tangents are used for linearization and Eshelby's tensor is calculated using the isotropic projection of the continuum tangent of the matrix phase.

Figure 5.10 shows the results of the model during a simple-shear deformation for an inclusion volume fraction of 0.2. For comparison, the austenite curve is also provided in the figure. At a first glance, it seems that all models are successful in predicting a harder response of the composite than single-phase austenite. As expected, Reuss model predicts the softest response, since it serves as a lower bound, as in the case of elastic composites.

It is observed that Mori-Tanaka, Bound-Interpolation, Lielens and Self-Consistent results are very similar. This is an expected outcome since for low volume fractions the Mori-Tanaka assumption holds quite well. It is interesting to see that these 


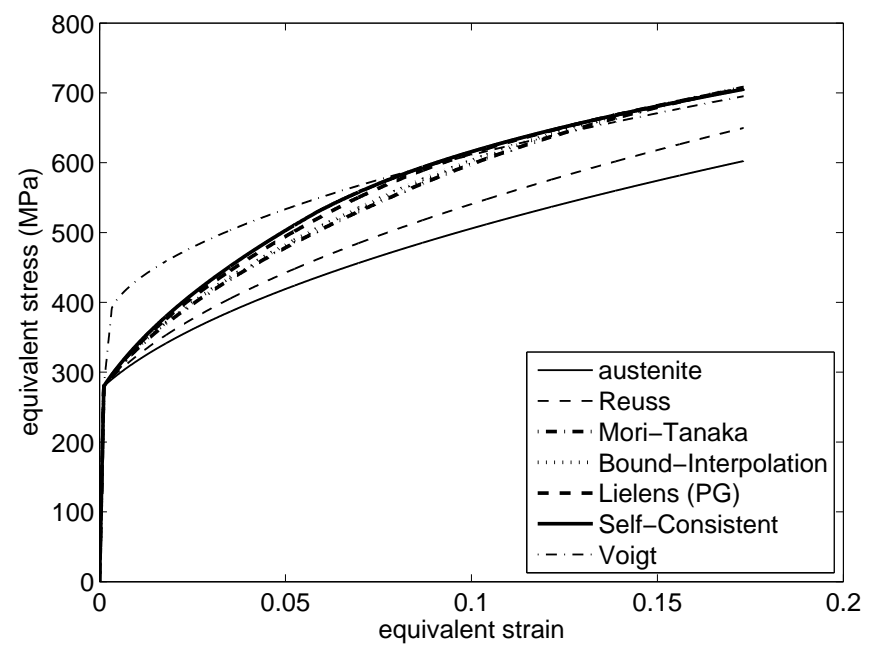

Figure 5.10: Stress-strain behavior of the composite with $20 \%$ volume fraction of inclusions.

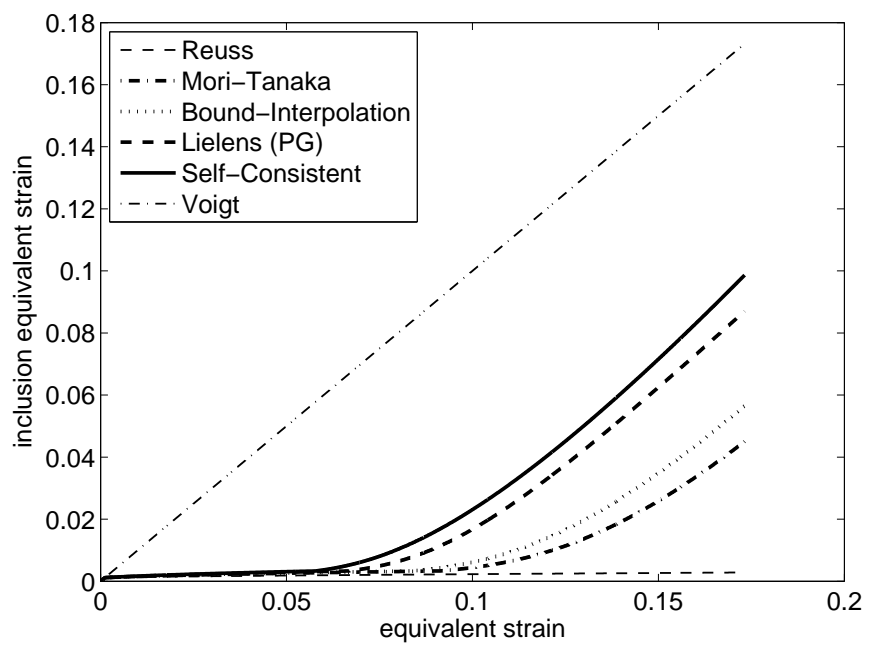

Figure 5.11: Strain concentration during deformation for $20 \%$ volume fraction of inclusions.

models merge with the Voigt model after a certain amount of deformation. Although this would never happen in an elastic calculation, for elastic-plastic problems this is possible and it does not lead to the conclusion that the models are as stiff as the Voigt model. 
In Figure 5.11, the equivalent strain that has accumulated in the inclusions is plotted against the total equivalent strain. This plot can be used to see the relative strain concentration behavior during the deformation by looking at the slope of the curves. It is observed that in the first stages, when martensite is still in the elastic regime, most of the strain is concentrated in austenite and all models successfully predict this. When martensite starts to deform plastically, it can be seen that martensite starts to experience as much strain increment as austenite, which is the Voigt assumption. This behavior can be explained by looking at the stress-strain curves of the phases. It is seen in Figure 5.9 that martensite does not show any significant strain hardening. Therefore, during the simulations when both phases are in the plastic regime, the instantaneous elastoplastic modulus of martensite is comparable to that of austenite which is the reason why the strain is almost equally distributed.

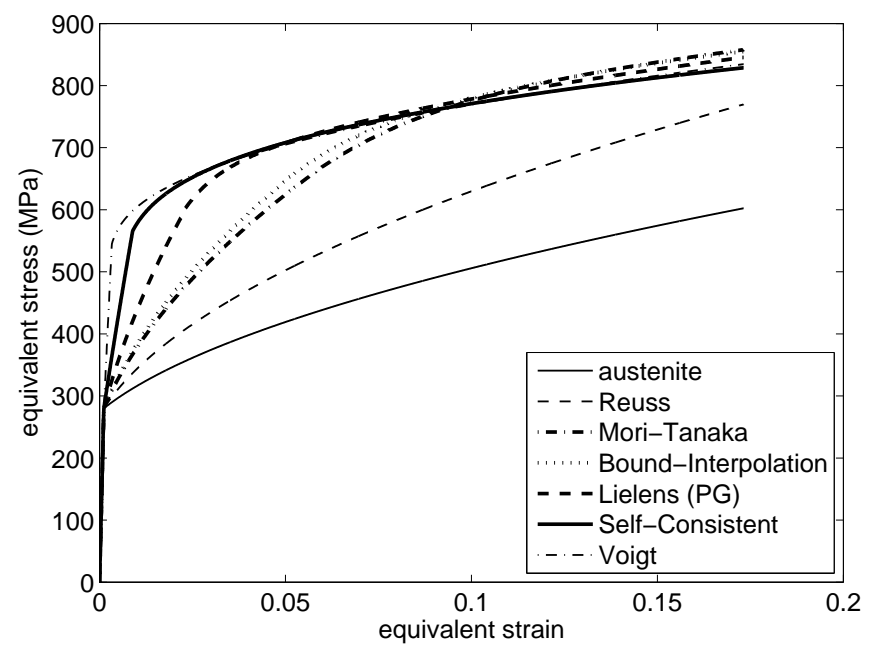

Figure 5.12: Stress-strain behavior of the composite with $50 \%$ volume fraction of inclusions.

Figures 5.12 and 5.13 show the results of the same simulation when the composite involves $50 \%$ of inclusions. Of course, as mentioned before, at this volume fraction the definition of the matrix and inclusion is ambiguous since both phases are expected to be interconnected. The results show the same tendency as the previous example; Reuss model gives the softest response, followed by Mori-Tanaka, Bound-Interpolation and Lielens. The observation repeats itself that the difference between the Voigt and Self-Consistent models are small. Lielens and Mori-Tanaka models are significantly softer compared to Self-Consistent.

On the other hand, it is very difficult to comment on the behavior of the composite at these volume fractions and the Self-Consistent model may not be very reliable in this case either. Although it is the one that represents the physics most accurately, it is the validity of the Mean-Field methods at these volume fractions that can cause inaccurate results. 


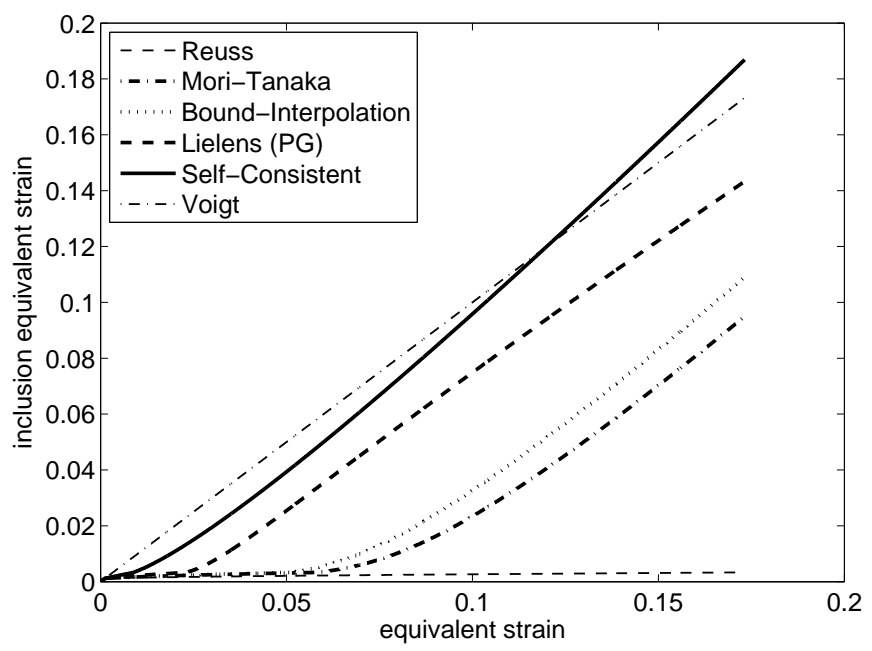

Figure 5.13: Strain concentration during deformation for $50 \%$ volume fraction of inclusions.

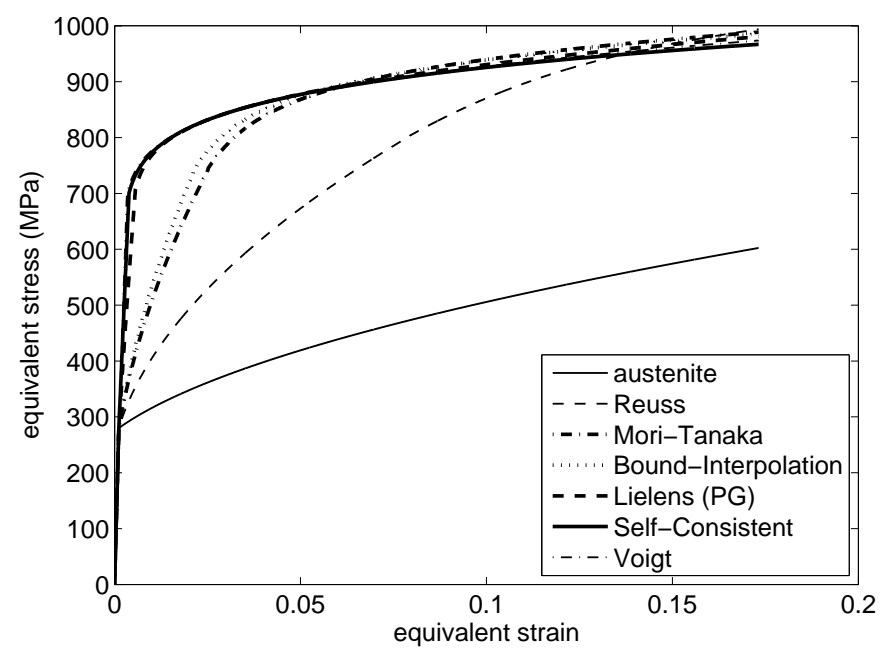

Figure 5.14: Stress-strain behavior of the composite with $80 \%$ volume fraction of inclusions.

Figures 5.14 and 5.13 show the results when the volume fraction of the inclusions is 0.8. The Reuss model is once again the softest, followed by Mori-Tanaka and BoundInterpolation. The Lielens, Self-Consistent and Voigt results are very close. MoriTanaka and Reuss models are expected to give inaccurate results in this case because the assumptions made in those models are not valid at high volume fractions. The 


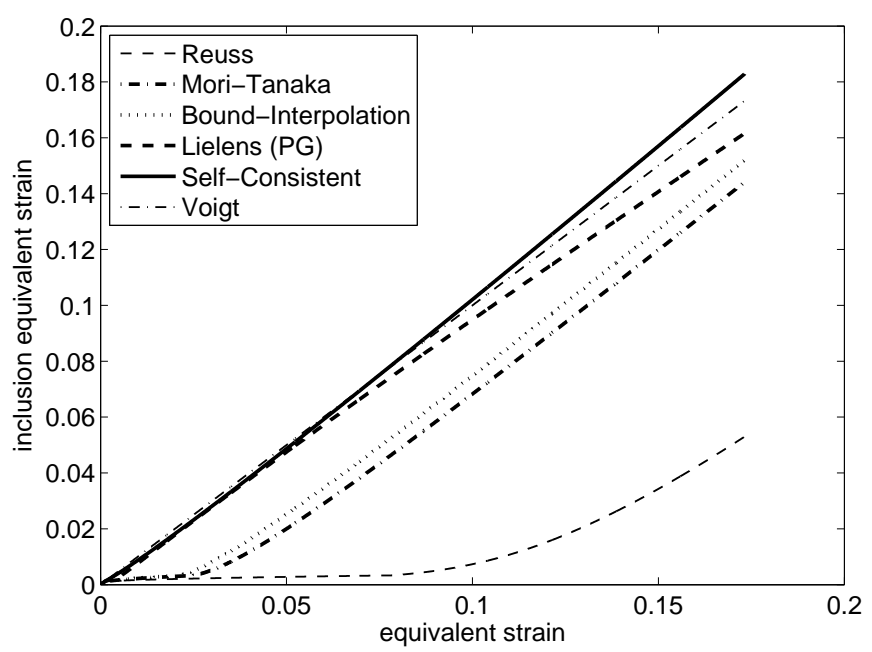

Figure 5.15: Strain concentration during deformation for $80 \%$ volume fraction of inclusions.

Self-Consistent model has no assumptions that rely on low volume fractions therefore it is expected to give the most accurate results. The results of the Lielens model approach the reverse Mori-Tanaka model which lies very close to the Self-Consistent model. On the other hand, the Voigt model also seems to give good results. The reason for this is that, as discussed above, the strain concentration tensor is close to unity in the plastic regime and for high volume fractions this regime is reached very fast.

In Figure 5.16, the evolution of the average stress and strain in the individual phases during deformation is plotted. The results shown are for the Self-Consistent model for $20 \%$ volume fraction of inclusions. Expectedly, it is observed that martensite is the load carrying phase whereas most of the strain accumulates in austenite. In the region where austenite is plastic and martensite is still elastic, it is visible that the strain concentrated in martensite is much lower compared to austenite. Beyond the yield point of martensite however, martensite starts to undergo comparable strain to austenite. This is because the instantaneous modulus of martensite is close to that of austenite.

The comparison of Figures 5.16 and 5.17 shows that the Lielens model with the proposed interpolation function matches quite accurately to the Self-Consistent model also in terms of the stress and strain partitioning. It has to be mentioned that there is a significant difference in computation time of the models, in favor of the Lielens model. Additionally, since the Self-Consistent model involves solution of a non-linear system of equations, care must be taken in setting up the system so that a globally convergent scheme is obtained. In some cases the Fixed-Point iteration method is insufficient in terms of searching for a solution therefore the scheme requires a better 

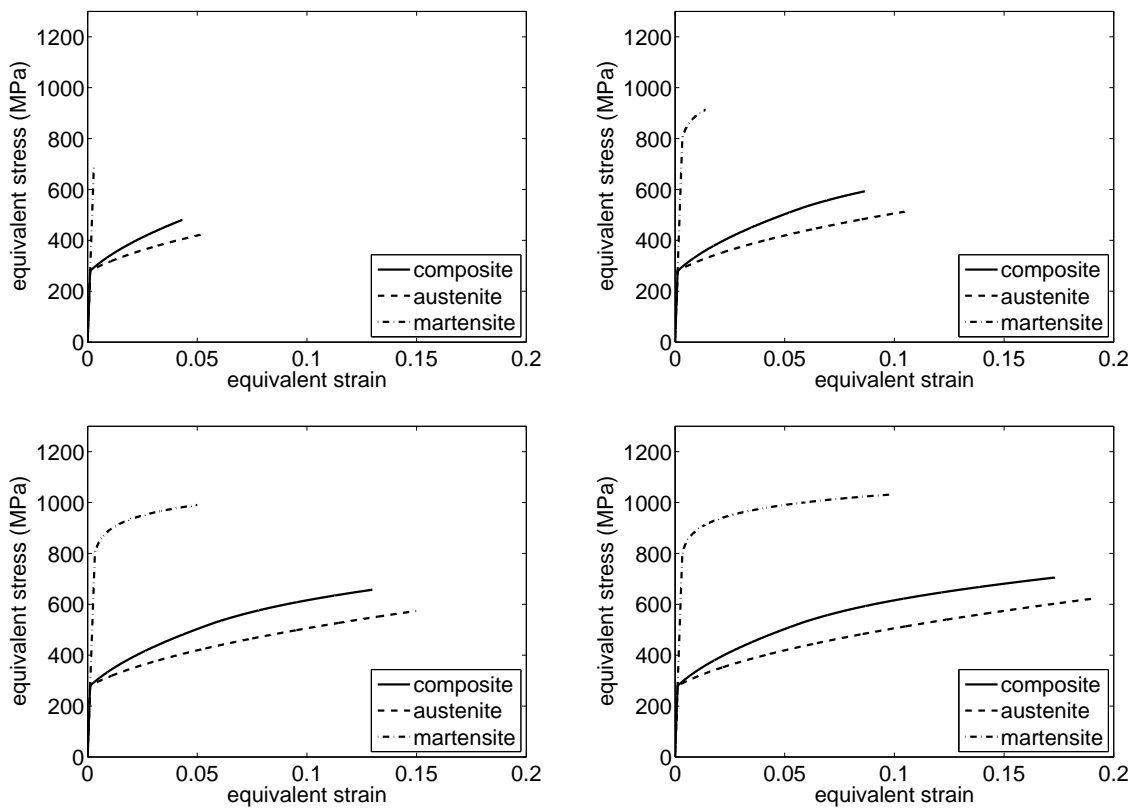

Figure 5.16: Partitioning of strain and stress in phases during deformation, as calculated by the Self-Consistent model for $20 \%$ volume fraction of inclusions.

solution algorithm.

\subsection{Summary and conclusions}

Metastable austenitic stainless steels are actually composite materials during the deformation process hence, a homogenization procedure was applied in order to construct the constitutive model on a physical basis. In this chapter, the details of the study on different homogenization algorithms was presented.

Since the aim of this study is to develop a macroscopic constitutive model, the homogenization procedures used must be accurate, robust and fast. The Mean-Field homogenization method was found to satisfy these requirements. The most common homogenization schemes within the Mean-Field method are Mori-Tanaka and SelfConsistent. Both schemes rely on the analytical solution provided by Eshelby for the strain concentration inside an inhomogeneity. The Mori-Tanaka scheme is based on the assumption that the volume fraction of the second phase particles is low. Due to the simplicity that this assumption introduces, this scheme is very robust and fast. It was also found to be very accurate for low volume fractions. Contrary to the Mori-Tanaka model, the Self-Consistent scheme does not have any restrictions on the volume fractions. On the other hand, it is an implicit scheme, requiring the solution of a non-linear system of equations. 

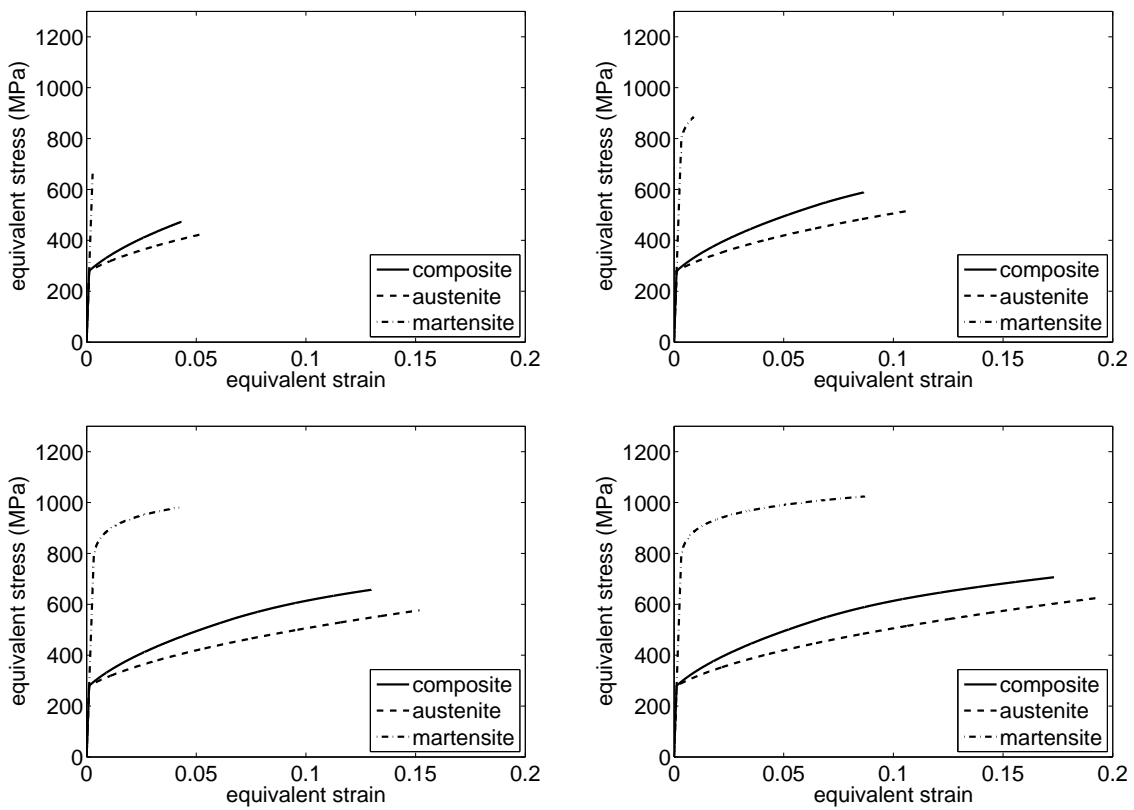

Figure 5.17: Partitioning of strain and stress in phases during deformation, as calculated by the Lielens (PG) model for $20 \%$ volume fraction of inclusions.

Another model based on the Mori-Tanaka approach was proposed by Lielens, that uses an interpolation between the forward and reverse Mori-Tanaka models. It was shown that by using a suitable interpolation function, it is possible to get results that are close to the Self-Consistent model for a larger volume fraction range.

Motivated by the Lielens model a new homogenization scheme was proposed which is based on the interpolation between the Reuss and Voigt approximations. This scheme is purely phenomenological and does not rely on Eshelby's solution. It was found that for elastic composites, the results are promising but the same is not true for the elastic-plastic composites.

The elastic homogenization schemes were generalized to the elastic-plastic problems by adopting a large-deformation formulation for strains and stresses and by linearizing the non-linear material response using an incremental formulation. The model results were compared and it was shown that the Lielens formulation with the proposed interpolation function gives results comparable to the Self-Consistent method. This scheme is explicit and was found to be very robust which make it suitable to be used in the constitutive model. In the next chapter the additional modifications needed for adopting the model for evolving volume fractions are discussed.

The results of the elastic-plastic simulations were compared with the Direct Finite Element method results in terms of the macroscopic response of the composite and a good correspondence was observed. Unfortunately, due to the difficulty in measuring 
the strains and stresses localized in the phases experimentally, it was not possible to verify the model results in terms of average strains and stresses in the phases. 


\section{Constitutive model}

In FE analysis, the mechanical behavior of materials is captured by models that provide the stress-strain relation for the material under consideration. These models incorporate the material behavior via the underlying yield, hardening as well as microstructural evolution functions. In this definition, the model is independent of the strain and stress measures used and only supplies material related information. To exploit the material models to their full extent, reliable definitions for the strain and stress must be made that take into account large deformations. There are a number of choices for these measures, which lead to different types of constitutive models. Therefore, the main purpose of the constitutive model is to compute the strain for a given deformation and use the material model to update the stress.

Additionally, FE formulations for large deformations result in a non-linear system of equations which can be solved by explicit or implicit algorithms. Implicit algorithms are generally based on the Newton-Raphson method, thus require calculation of the stiffness matrix at each iteration. Consequently, the constitutive model must provide a tangent modulus that relates the increment of stress to the increment of strain.

The chapter starts with a very brief summary of the Updated-Lagrangian FE method that gives insight on how the constitutive model is related to the FE formulation. Following that, the procedure of the decomposition of the deformation into elastic, plastic and transformation parts is introduced. The chapter continues with the details of the stress-update algorithm and the derivation of the material tangent modulus. Finally, results of simulations are presented to demonstrate the capabilities of the model.

\subsection{General concepts in constitutive modeling}

In this section a general introduction to the non-linear FE method for large deformations is presented. The details of the formulations can be found for example in $[2,37,92]$ and related information on continuum mechanics can be found in $[9,55]$. 
In metal forming operations, the inertia terms in the momentum equation are generally neglected, which leads to the equilibrium equation:

$$
\sigma \cdot \nabla=0 .
$$

Equation (6.1) can be written in a weak formulation by multiplying with the weight functions, applying the divergence theorem and integrating by parts:

$$
\int_{\Omega} \mathbf{w} \nabla: \sigma \mathrm{d} V=\int_{\Gamma} \mathbf{w} \cdot \mathbf{t} \mathrm{d} A
$$

where $\mathbf{w}$ is the vector of weight functions and $\mathbf{t}$ is the traction on the boundary surface. Equation (6.2) is also known as the virtual power equation. The terms on the left hand side (LHS) and the right hand side (RHS) are referred to as the internal and external power, respectively.

In an Updated-Lagrangian formulation, usually the rate form of the virtual power equation is utilized. Therefore, it is necessary to differentiate Equation (6.2) with respect to time. For the derivation it is useful to evaluate the integral in the undeformed configuration, which makes the coordinates independent of time leaving the stress-rate as the only time-dependent term. By changing to the reference coordinates, the internal power in Equation (6.2) becomes:

$$
P^{\text {int }}=\int_{\Omega_{0}} \mathbf{w} \nabla_{0}: \mathbf{P} \mathrm{d} V_{0}
$$

where

$$
\mathbf{P}=J \mathbf{F}^{-1} \cdot \boldsymbol{\sigma}
$$

is the Nominal stress.

The rate of Nominal stress can be obtained by the following:

$$
\begin{aligned}
\boldsymbol{\sigma} & =\frac{1}{J} \mathbf{F} \cdot \mathbf{P}, \\
\Rightarrow \dot{\boldsymbol{\sigma}} & =\frac{1}{J}\left[(\dot{\mathbf{F}} \cdot \mathbf{P}+\mathbf{F} \cdot \dot{\mathbf{P}})-\frac{\dot{J}}{J}(\mathbf{F} \cdot \mathbf{P})\right], \\
& =\mathbf{L} \cdot \boldsymbol{\sigma}+\frac{1}{J} \mathbf{F} \cdot \dot{\mathbf{P}}-\operatorname{tr}(\mathbf{L}) \boldsymbol{\sigma}, \\
\Rightarrow \dot{\mathbf{P}} & =J \mathbf{F}^{-1} \cdot(\dot{\boldsymbol{\sigma}}-\mathbf{L} \cdot \boldsymbol{\sigma}+\operatorname{tr}(\mathbf{L}) \boldsymbol{\sigma}),
\end{aligned}
$$

where the relations $\mathbf{L}=\dot{\mathbf{F}} \cdot \mathbf{F}^{-1}$ and $\operatorname{tr}(\mathbf{L})=\frac{j}{J}$ have been utilized. The rate of internal power is then given by:

$$
\dot{P}^{\text {int }}=\int_{\Omega} \mathbf{w} \nabla:(\dot{\boldsymbol{\sigma}}-\mathbf{L} \cdot \boldsymbol{\sigma}+\operatorname{tr}(\mathbf{L}) \boldsymbol{\sigma}) \mathrm{d} V
$$

This result can be further simplified by introducing the Truesdell rate of stress in the equation, which reads:

$$
\stackrel{\circ}{\boldsymbol{\sigma}}=\dot{\boldsymbol{\sigma}}-\mathbf{L} \cdot \boldsymbol{\sigma}-\boldsymbol{\sigma} \cdot \mathbf{L}^{\mathrm{T}}+\operatorname{tr}(\mathbf{L}) \boldsymbol{\sigma} .
$$


Hence:

$$
\dot{P}^{\text {int }}=\int_{\Omega} \mathbf{w} \nabla:\left(\stackrel{\circ}{\boldsymbol{\sigma}}+\boldsymbol{\sigma} \cdot \mathbf{L}^{\mathrm{T}}\right) \mathrm{d} V
$$

It is common practice to use the Jaumann rate of Cauchy stress tensor in the constitutive models. Furthermore, the velocity gradient is decomposed into a symmetric and an anti-symmetric part as:

$$
\mathbf{D}=\frac{1}{2}\left(\mathbf{L}^{\mathrm{T}}+\mathbf{L}\right), \quad \mathbf{W}=\frac{1}{2}\left(\mathbf{L}^{\mathrm{T}}-\mathbf{L}\right)
$$

where $\mathbf{D}$ and $\mathbf{W}$ are the deformation rate and spin tensors, respectively. The relation between the Jaumann rate of the Cauchy stress and the deformation rate is given in the form:

$$
\stackrel{\nabla}{\boldsymbol{\sigma}}=\mathcal{C}^{\mathrm{J}}: \mathbf{D}
$$

where $\mathcal{C}^{\mathrm{J}}$ is the material tangent given in Jaumann terms. This tangent can be converted easily to the Truesdell terms as:

$$
C_{i j k l}^{\mathcal{T}}=C_{i j k l}^{\mathrm{J}}-\frac{1}{2}\left(\delta_{i k} \sigma_{j l}+\delta_{i l} \sigma_{j k}+\delta_{j k} \sigma_{i l}+\delta_{j l} \sigma_{i k}\right)+\sigma_{i j} \delta_{k l} .
$$

Substituting the material tangent, Equation (6.8) can be written as:

$$
\dot{P}^{\text {int }}=\int_{\Omega} \mathbf{w} \nabla:\left(\mathcal{C}^{\mathcal{T}}: \mathbf{D}+\boldsymbol{\sigma} \cdot \mathbf{L}^{\mathrm{T}}\right) \mathrm{d} V
$$

In the FE method, the virtual power equation is further discretized with the FE interpolation functions (see for example [14, 38]). During the implicit solution process, at each iteration the constitutive model is called with the trial deformation and it returns the corresponding increment of stress, which is used to check if equilibrium is satisfied. Additionally, the constitutive model supplies the material tangent relating the increment of stress to the increment of strain. This tangent is used in building up the stiffness matrix that will be used to predict the next iterative solution.

\subsection{Decomposition of strain}

For materials undergoing large deformations it is observed that the total deformation is made up of a reversible and an irreversible part. The reversible part is usually referred to as the elastic part and the irreversible part as the plastic part. The elastic part is responsible for the increase in stress and the plastic part represents the unrecoverable strain.

In hyperelastic-plastic formulations, stress is related to the elastic strain via a stored energy function which ensures that the work is independent of the deformation path [2]. This results in a non-linear relation between the elastic strain and the stress. In case of rubber-like materials, the extent of elastic deformation is very large and the stress-strain response is in the non-linear regime. However, in the case of metals, the 
attainable maximum elastic strain is very small, therefore the non-linear regime is not reached. Therefore, for metals, a hypoelastic plastic formulation is sufficient to describe the process $[2,37,78]$.

In hypoelastic-plastic formulations, the relation between stress and the elastic strain is assumed to be constant. The total deformation rate is decomposed additively into elastic and plastic parts. The plastic deformation rate is defined by the plasticity parameter and the yielding direction.

In a composite material, the deformation rate is partitioned between the two phases according to the strain concentration tensor. Each phase behaves with its own material model and the average response is calculated by the homogenization procedures defined in the previous chapter.

Furthermore, as discussed in Chapter 2, metastable austenitic stainless steels undergo transformation during the deformation which causes an additional inelastic strain. To incorporate all the different deformations, the following decomposition is introduced.

The total deformation rate is first decomposed into an elastic-plastic, $\mathbf{D}^{\mathrm{ep}}$, and a transformation, $\mathbf{D}^{\text {tr }}$, part:

$$
\mathbf{D}=\mathbf{D}^{\mathrm{ep}}+\mathbf{D}^{\mathrm{tr}}
$$

where, the elastic-plastic part stands for all the deformation that is not caused by the transformation.

The elastic-plastic deformation enters the homogenization algorithm in which it is further partitioned as:

$$
\mathbf{D}^{\mathrm{ep}}=f_{1} \mathbf{D}_{1}+f_{0} \mathbf{D}_{0}
$$

where for notational convenience the averaging operator is dropped, i.e. $\mathbf{D} \equiv\langle\mathbf{D}\rangle_{\omega}$ hence, $\mathbf{D}_{1}\left(\equiv\langle\mathbf{D}\rangle_{\omega_{1}}\right)$ and $\mathbf{D}_{0}\left(\equiv\langle\mathbf{D}\rangle_{\omega_{0}}\right)$ are the strains concentrated in martensite and austenite, respectively.

Finally, each phase is assumed to follow conventional elastic-plastic behavior and the deformation rates are decomposed into elastic and plastic parts, as will be described in the next section.

\subsection{Single phase constitutive model}

Austenite and martensite phases are assumed to follow a hypoelastic-plastic type constitutive relation. J2-flow plasticity for each phase is assumed, implying associated plasticity with a Von-Mises type yield surface. Furthermore, isotropic hardening for each phase is assumed. All these assumptions make it possible to utilize the radial return mapping algorithm for updating the stress. Details of the formulations presented below can be found in for example [16, 43, 55]. 


\subsubsection{Hypoelastic-plastic constitutive model}

In the hypoelastic-plastic formulation the total deformation is decomposed into an elastic and plastic part as

$$
\mathbf{D}=\mathbf{D}^{\mathrm{e}}+\mathbf{D}^{\mathrm{p}}
$$

Stress rate depends only on the elastic deformation, therefore:

$$
\stackrel{\nabla}{\boldsymbol{\sigma}}=\mathcal{C}^{\mathrm{J}, \mathrm{el}}: \mathbf{D}^{\mathrm{e}}=\mathcal{C}^{\mathrm{J}, \mathrm{el}}:\left(\mathbf{D}-\mathbf{D}^{\mathrm{p}}\right)
$$

where $\mathcal{C}^{\mathrm{J}, \mathrm{el}}$ is the elasticity tensor given by:

$$
\mathcal{C}^{\mathrm{J}, \mathrm{el}}=\kappa \mathbf{I} \otimes \mathbf{I}+2 \mu\left(\mathcal{I}-\frac{1}{3} \mathbf{I} \otimes \mathbf{I}\right)
$$

and $\kappa$ and $\mu$ are the bulk and shear modulus, respectively.

The plastic flow is characterized by the plastic flow rate, $\dot{\lambda}$, and the plastic flow direction, $\mathbf{r}$, which is a function of stress and hardening parameters, $\mathbf{q}$ as:

$$
\mathbf{D}^{\mathrm{p}}=\dot{\lambda} \mathbf{r}(\boldsymbol{\sigma}, \mathbf{q})
$$

The plastic flow direction stems from the plastic flow potential, $\psi$, as:

$$
\mathbf{r}=\frac{\partial \psi}{\partial \boldsymbol{\sigma}}
$$

In case of time-independent plastic deformation, a yield function is defined with the same parameters as the plastic flow potential:

$$
\phi(\boldsymbol{\sigma}, \mathbf{q})=0
$$

which characterizes the yield condition based on a given stress state and hardening variables.

If the derivative of the yield function with respect to stress (normal to the yield surface) is assumed to be equal to the plastic flow direction, i.e.

$$
\frac{\partial \psi}{\partial \boldsymbol{\sigma}}=\frac{\partial \phi}{\partial \boldsymbol{\sigma}},
$$

then the resulting plastic flow is said to be associative.

The following plastic flow (Kuhn-Tucker) conditions must hold during deformation of a material:

$$
\dot{\lambda} \geq 0, \quad \phi \leq 0, \quad \dot{\lambda} \phi=0 .
$$

The first condition states that the plastic flow cannot be negative, meaning that the plastic flow cannot be undone. The second condition says that the yield function cannot be positive in a converged solution, requiring the stress to always remain on or inside the yield surface. Finally, the third condition implies that, if plastic flow 
takes place the stress must be on the yield surface or if stress is inside the yield surface, there cannot be any plastic flow.

The consistency condition parallels the plastic flow conditions and states that the rate of change of the yield function must always remain 0 :

$$
\dot{\phi}=\frac{\partial \phi}{\partial \boldsymbol{\sigma}}: \dot{\boldsymbol{\sigma}}+\frac{\partial \phi}{\partial \mathbf{q}} \cdot \dot{\mathbf{q}}=0 .
$$

This condition implies that when plastic flow takes place, the stresses must remain on the yield surface.

Hardening parameters generally depend only on the equivalent plastic strain, hence:

$$
\dot{\mathbf{q}}=\dot{\lambda} \mathbf{h}(\boldsymbol{\sigma}, \mathbf{q})
$$

where $\mathbf{h}$ is the vector of hardening variables.

It is shown in [37] that the stress rate term in Equation (6.23) can be replaced by the Jaumann rate of stress. Therefore, using Equations (6.16), (6.18) and (6.23), the consistency condition becomes:

$$
\dot{\phi}=\frac{\partial \phi}{\partial \boldsymbol{\sigma}}: \mathcal{C}^{\mathrm{J}, \mathrm{el}}:(\mathbf{D}-\dot{\lambda} \mathbf{r})+\dot{\lambda} \frac{\partial \phi}{\partial \mathbf{q}} \cdot \mathbf{h}=0
$$

From the above, the plastic flow rate can be solved as:

$$
\dot{\lambda}=\frac{\frac{\partial \phi}{\partial \boldsymbol{\sigma}}: \mathcal{C}^{\mathrm{J}, \mathrm{el}}: \mathbf{D}}{-\frac{\partial \phi}{\partial \mathbf{q}} \cdot \mathbf{h}+\frac{\partial \phi}{\partial \boldsymbol{\sigma}}: \mathcal{C}^{\mathrm{J}, \mathrm{el}}: \mathbf{r}}
$$

Substituting Equation (6.26) into (6.16) using (6.18), the material tangent that relates the rate of stress to the rate of deformation is obtained as:

$$
\mathcal{C}^{\mathrm{J}}=\mathcal{C}^{\mathrm{J}, \mathrm{el}}-\frac{\left(\mathcal{C}^{\mathrm{J}, \mathrm{el}}: \mathbf{r}\right) \otimes\left(\frac{\partial \phi}{\partial \boldsymbol{\sigma}}: \mathcal{C}^{\mathrm{J}, \mathrm{el}}\right)}{-\frac{\partial \phi}{\partial \mathbf{q}} \cdot \mathbf{h}+\frac{\partial \phi}{\partial \boldsymbol{\sigma}}: \mathcal{C}^{\mathrm{J}, \mathrm{el}}: \mathbf{r}}
$$

This tangent is referred to as the continuum tangent because it is given in a rate form. To be consistent with the solution algorithm this expression must be given in incremental form, which depends on the algorithm used for the stress update.

\subsubsection{J2-Flow plasticity}

For metals in general, the plastic flow is associative and pressure independent. The yield condition therefore, is based on the deviatoric part of the stress tensor and expressed with the Von Mises yield surface. These assumptions introduce certain simplifications in the formulations and solution algorithms. The formulations presented below are based on an isotropic strain hardening material. 
The yield function is expressed as:

$$
\phi=\sigma^{\mathrm{eq}}-\sigma^{\mathrm{y}}=\left(\frac{3}{2} \boldsymbol{\sigma}^{\prime}: \boldsymbol{\sigma}^{\prime}\right)^{\frac{1}{2}}-\sigma^{\mathrm{y}}=0
$$

where $\sigma^{\text {eq }}$ is the Von Mises equivalent stress, $\boldsymbol{\sigma}^{\prime}$ is the deviatoric part of the stress tensor and $\sigma^{\mathrm{y}}$ is the yield stress determined by a uniaxial tensile experiment.

The derivative of the yield function with respect to stress, i.e. normal to the yield surface, is given by:

$$
\mathbf{N}=\frac{3}{2} \frac{\boldsymbol{\sigma}^{\prime}}{\sigma^{\mathrm{eq}}}
$$

and since associated plasticity is assumed:

$$
\mathbf{r}=\mathbf{N}
$$

In case of isotropic strain hardening, flow stress is a function of the total equivalent plastic strain. Consequently:

$$
\frac{\partial \phi}{\partial \mathbf{q}}=-\frac{\mathrm{d} \sigma^{\mathrm{y}}}{\mathrm{d} \varepsilon^{\mathrm{p}}}
$$

where $\varepsilon^{\mathrm{p}}$ is the total equivalent plastic strain.

Using the above, the plastic rate parameter equation simplifies to:

$$
\dot{\lambda}=\frac{\mathbf{N}: \mathcal{C}^{\mathrm{J}, \mathrm{el}}: \mathbf{D}}{\frac{\mathrm{d} \sigma^{\mathrm{y}}}{\mathrm{d} \varepsilon^{p}}+\mathbf{N}: \mathcal{C}^{\mathrm{J}, \mathrm{el}}: \mathbf{N}} .
$$

Due to the fact that $\mathbf{N}$ is deviatoric, the following relations are obtained:

$$
\begin{gathered}
\mathcal{C}^{\mathrm{J}, \mathrm{el}}: \mathbf{N}=2 \mu \mathbf{N}, \\
\mathbf{N}: \mathcal{C}^{\mathrm{J}, \mathrm{el}}: \mathbf{N}=3 \mu .
\end{gathered}
$$

Finally, the continuum material tangent for J2-flow plasticity is obtained as:

$$
\mathcal{C}^{\mathrm{J}}=\mathcal{C}^{\mathrm{J}, \mathrm{el}}-\frac{4 \mu^{2}}{\frac{\mathrm{d} \sigma^{\mathrm{y}}}{\mathrm{d} \varepsilon^{p}}+3 \mu} \mathbf{N} \otimes \mathbf{N}
$$

\subsubsection{Stress update algorithm}

The equations in the constitutive model are in rate form. However for the numerical calculations, an incremental form is required. For a given incremental deformation, the stress is calculated by the stress-update algorithm. The primary objective of the algorithm is to make sure that the calculated stress satisfies the consistency condition. The Backward-Euler algorithm is generally employed in solving the equations, which manifests that the consistency condition is satisfied by the updated variables at the end of the step. Since the variables at the end of the step are yet to be determined, this method is implicit and generally requires solution of non-linear equations. The algorithm generally consists of an elastic predictor step where, the deformation is 
considered as purely elastic and the yield condition is checked. If the calculated trial stress is outside the yield surface then a plastic corrector step is utilized where the equations for the normal to the yield surface and the plastic flow rate are solved simultaneously. Based on the geometrical interpretation of the corrector stage, this step is usually referred to as return-mapping.

For the case of J2-flow plasticity, the algorithm can be simplified considerably. Since it is assumed that the plastic flow direction is always normal to the yield surface, in the corrector stage the normal to the yield surface does not change. Geometrically this implies that the stress is returned to the yield surface radially and the algorithm is called radial-return. Error analysis of different return mapping algorithms can be found in [54].

The only unknown to be solved for during the return is the plastic flow rate, which usually reduces to solving a non-linear scalar equation numerically. The algorithm is best visualized on the deviatoric plane in the principal stress space, as shown in Figure 6.1.

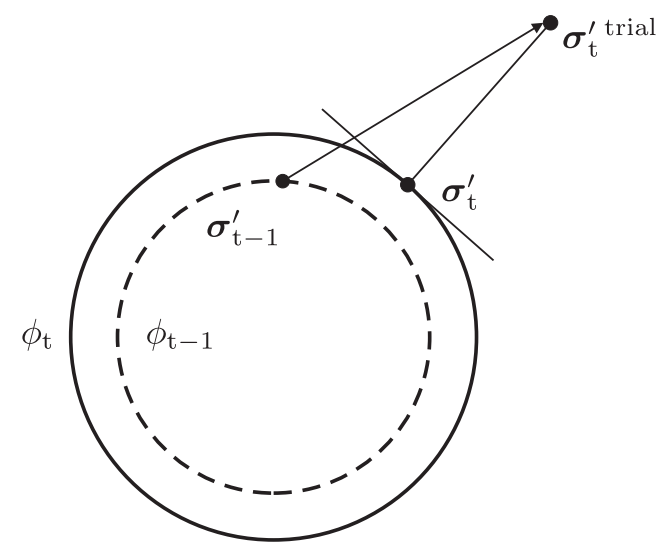

Figure 6.1: Illustration of the radial return mapping algorithm.

In the constitutive model, the Jaumann rate of the Cauchy stress tensor is used. During a finite time step, the stress must be updated objectively by taking into account the incremental rotation. The updated stress is given by [2]:

$$
\boldsymbol{\sigma}_{t}=\mathbf{Q}_{t} \cdot \boldsymbol{\sigma}_{t-1} \cdot \mathbf{Q}_{t}^{\mathrm{T}}+\Delta t \stackrel{\nabla}{\boldsymbol{\sigma}}
$$

where $\mathbf{Q}$ is the incremental rotation tensor, that is approximated using the spin tensor (see Equation (6.9)) as:

$$
\mathbf{Q} \approx\left(\mathbf{I}-\frac{1}{2} \mathbf{W} \Delta t\right)^{-1} \cdot\left(\mathbf{I}+\frac{1}{2} \mathbf{W} \Delta t\right)
$$

The normal to the yield surface is calculated in the predictor stage as:

$$
\mathbf{N}=\mathbf{N}^{\text {trial }}=\frac{3}{2} \frac{\boldsymbol{\sigma}^{\text {trial }}}{\sigma^{\text {eq,trial }}} .
$$


In the corrector stage the stress is simply taken back to the yield surface determined using the plastic flow rate:

$$
\boldsymbol{\sigma}_{t}=\boldsymbol{\sigma}_{t}^{\text {trial }}-2 \mu \Delta \lambda \mathbf{N}
$$

The plastic flow increment, $\Delta \lambda$, depends only on the hardening behavior of the material and in case of isotropic hardening, it can be found by the solution of a scalar equation.

The material tangent that is consistent with the algorithm described above is given by $[2,16,78]$ :

$$
\mathcal{C}^{\mathrm{J}, \mathrm{alg}}=\mathcal{C}^{\mathrm{J}}-\frac{4 \mu^{2} \Delta \lambda}{\sigma^{\text {eq,trial }}}\left(\frac{3}{2}\left(\mathcal{I}-\frac{1}{3} \mathbf{I} \otimes \mathbf{I}\right)+\mathbf{N} \otimes \mathbf{N}\right)
$$

\subsection{Transformation}

In Chapter 4, a transformation function was introduced that dictates how much martensite is expected to form under a given stress. First, this equation must be cast into a rate form to be used in the constitutive model. Furthermore, transformation causes two important physical phenomena that need to be considered: the appearance of new martensite particles within a parent austenite lattice and the transformation strain.

\section{Rate of transformation}

The amount of martensite as a function of the stress tensor was established in Chapter 4 as:

$$
f^{\alpha^{\prime}}=1-\left[1+(r-1)\left(\frac{U^{\max }}{\Delta G^{\mathrm{cr}}+\Delta G^{\text {off }}}\right)^{m}\right]^{\frac{1}{1-r}}
$$

To obtain the rate form of Equation (4.25), first, the chain rule is applied which gives:

$$
\dot{f}^{\alpha^{\prime}}=\frac{\mathrm{d} f^{\alpha^{\prime}}}{\mathrm{d} \boldsymbol{\sigma}}: \dot{\boldsymbol{\sigma}}=\frac{\mathrm{d} f^{\alpha^{\prime}}}{\mathrm{d} U^{\max }} \frac{\mathrm{d} U^{\max }}{\mathrm{d} \boldsymbol{\sigma}}: \dot{\boldsymbol{\sigma}} .
$$

The first term on the RHS can be obtained trivially by scalar differentiation:

$$
\frac{\mathrm{d} f^{\alpha^{\prime}}}{\mathrm{d} U^{\max }}=\frac{m}{U^{\max }}\left(\frac{U^{\max }}{\Delta G^{\mathrm{cr}}+\Delta G^{\text {off }}}\right)^{m}\left(1-f^{\alpha^{\prime}}\right)^{r} .
$$

Computation of the second term on the RHS is more elaborate. Consider, first, the equation for the maximum mechanical driving force, $U^{\max }$ :

$$
U^{\max }=\sum_{j} \sigma_{j}^{*} \lambda_{j}
$$


where $\sigma_{j}^{*}$ is the $j^{\text {th }}$ eigenvalue of the stress tensor (i.e. principal stress). Differentiation of this term with respect to stress gives,

$$
\frac{\mathrm{d} U^{\max }}{\mathrm{d} \sigma}=\sum_{j} \frac{\mathrm{d} \sigma_{j}^{*}}{\mathrm{~d} \boldsymbol{\sigma}} \lambda_{j}
$$

To continue with the computation, the variation of the principal stresses with respect to the components of the stress tensor is required. This can be achieved by considering the characteristic polynomial of the stress tensor:

$$
\left(\boldsymbol{\sigma}-\sigma_{j}^{*} \mathbf{I}\right) \cdot \mathbf{e}_{j}=0
$$

where $\mathbf{e}_{j}$ is the $j^{\text {th }}$ eigenvector of the tensor. Variation of this equation gives:

$$
\left(\mathrm{d} \boldsymbol{\sigma}-\mathrm{d} \sigma_{j}^{*} \mathbf{I}\right) \cdot \mathbf{e}_{j}+\left(\boldsymbol{\sigma}-\sigma_{j}^{*} \mathbf{I}\right) \cdot \mathrm{d} \mathbf{e}_{j}=0
$$

which can be pre-multiplied with $\mathbf{e}_{j}$ to yield:

$$
\mathbf{e}_{j} \cdot\left(\mathrm{d} \boldsymbol{\sigma}-\mathrm{d} \sigma_{j}^{*} \mathbf{I}\right) \cdot \mathbf{e}_{j}=0
$$

since the second term vanishes due to Equation (6.43).

Equation (6.45) can be rearranged to give

$$
\frac{\mathrm{d} \sigma_{j}^{*}}{\mathrm{~d} \boldsymbol{\sigma}}=\mathbf{e}_{j} \otimes \mathbf{e}_{j}
$$

where the relation $\mathbf{e}_{j} \cdot \mathbf{e}_{j}=1$ is utilized. Employing this result in Equation (6.42) gives,

$$
\frac{\mathrm{d} U^{\max }}{\mathrm{d} \boldsymbol{\sigma}}=\sum_{j} \lambda_{j} \mathbf{e}_{j} \otimes \mathbf{e}_{j}
$$

Combining the expressions obtained for the first and the second terms on the RHS of Equation (6.41) and considering that the stress that drives the transformation is the one that is resolved in austenite gives:

$$
\frac{\mathrm{d} f^{\alpha^{\prime}}}{\mathrm{d} \boldsymbol{\sigma}_{0}}=\frac{m}{U^{\max }}\left(\frac{U^{\max }}{\Delta G^{\mathrm{cr}}+\Delta G^{\mathrm{off}}}\right)^{m}\left(1-f^{\alpha^{\prime}}\right)^{r}\left(\sum_{j} \lambda_{j} \mathbf{e}_{j} \otimes \mathbf{e}_{j}\right) .
$$

Finally, the rate of transformation is calculated as:

$$
\dot{f}^{\alpha^{\prime}}=\frac{m}{U^{\max }}\left(\frac{U^{\max }}{\Delta G^{\mathrm{cr}}+\Delta G^{\text {off }}}\right)^{m}\left(1-f^{\alpha^{\prime}}\right)^{r}\left(\sum_{j} \lambda_{j} \mathbf{e}_{j} \otimes \mathbf{e}_{j}\right): \dot{\boldsymbol{\sigma}}_{0} .
$$




\section{Transformation strain}

An explicit expression for the transformation strain was established in Chapter 4:

$$
\mathbf{D}^{\operatorname{tr}}=\dot{f}^{\alpha^{\prime}}\left(T \mathbf{N}_{0}+\frac{\delta V}{3} \mathbf{I}\right) \equiv \dot{f}^{\alpha^{\prime}} \mathbf{T}
$$

where, for simplicity in notation, the tensor $\mathbf{T}$ is introduced.

Substituting Equation (6.49) for the transformation rate gives:

$$
\mathbf{D}^{\operatorname{tr}}=\frac{\mathrm{d} \dot{f}^{\alpha^{\prime}}}{\mathrm{d} \boldsymbol{\sigma}_{0}}: \dot{\boldsymbol{\sigma}_{0}} \mathbf{T} .
$$

Since martensite fraction is only a function of the invariants of the stress tensor, only a non-rotational change in the stress tensor will cause transformation. Therefore, the rate of austenite stress can be replaced by the Jaumann rate which is given by:

$$
\stackrel{\nabla}{\sigma}_{0}=\mathcal{C}_{0}^{\mathrm{J}, \mathrm{alg}}: \mathbf{D}_{0}=\mathcal{C}_{0}^{\mathrm{J}, \mathrm{alg}}: \mathcal{A}_{0}:\left(\mathbf{D}-\mathbf{D}^{\mathrm{tr}}\right)
$$

where $\mathcal{A}_{0}$ is the austenite strain concentration tensor (see Chapter 5).

Using this result in Equation (6.49) and after some algebra:

$$
\dot{f}^{\alpha^{\prime}}=\frac{\frac{\mathrm{d} f^{\alpha^{\prime}}}{\mathrm{d} \boldsymbol{\sigma}_{0}}: \mathcal{C}_{0}^{\mathrm{J}, \text { alg }}: \mathcal{A}_{0}}{1+\frac{\mathrm{d} f^{\alpha^{\prime}}}{\mathrm{d} \boldsymbol{\sigma}_{0}}: \mathcal{C}_{0}^{\mathrm{J}, \mathrm{alg}}: \mathcal{A}_{0}: \mathbf{T}}: \mathbf{D}
$$

is obtained which relates the rate of transformation to the total deformation rate. This result allows the following expression for the transformation strain:

$$
\mathbf{D}^{\mathrm{tr}}=\frac{\mathbf{T} \otimes \frac{\mathrm{d} f^{\alpha^{\prime}}}{\mathrm{d} \boldsymbol{\sigma}_{0}}: \mathcal{C}_{0}^{\mathrm{J}, \text { alg }}: \mathcal{A}_{0}}{1+\frac{\mathrm{d} f^{\alpha^{\prime}}}{\mathrm{d} \boldsymbol{\sigma}_{0}}: \mathcal{C}_{0}^{\mathrm{J}, \mathrm{alg}}: \mathcal{A}_{0}: \mathbf{T}}: \mathbf{D}
$$

\section{Dilution}

The transformation of austenite to martensite causes the mechanical properties of the material to change locally. When austenite has an average strain and a martensite plate forms inside it, at the first instant, equilibrium is disturbed since the superimposed strain causes a different stress in martensite. When the mismatch of stresses on the boundaries is relaxed, equilibrium is satisfied again resulting in a localized stress field. It is very difficult to find the stress distribution in the final geometry, however it is intuitively clear that the newly formed particle will not have the average stress of the other martensite particles that already exist in the material. To solve this problem a simple approach is followed where the average stress in the new martensite particle is assumed to be equal to the average stress in the austenite. This causes the overall average stress in martensite to be diluted due to the presence 
of new low-stressed particles. In mathematical terms, the rate of stress in martensite becomes:

$$
\stackrel{\nabla}{\sigma}_{1}=\mathcal{C}_{1}^{\mathrm{J}, \mathrm{el}}: \mathbf{D}_{1}^{\mathrm{e}}+\frac{\dot{f}^{\alpha^{\prime}}}{f^{\alpha^{\prime}}}\left(\boldsymbol{\sigma}_{0}-\boldsymbol{\sigma}_{1}\right), \quad f^{\alpha^{\prime}}>0
$$

The additional dilution term in the rate of stress in martensite also ensures that the martensite particles do not get loaded before they appear. In other words, the average stress in martensite, before there is any martensite (i.e. $f^{\alpha^{\prime}}=0$ ), is kept equal to the average stress in austenite.

\subsection{Stress integration and material tangent}

The rate equations introduced in the previous sections are solved using a Backward Euler (fully implicit) algorithm. This implies that all the time dependent variables are calculated at the end of the step. Therefore the set of equations:

$$
\begin{aligned}
\mathbf{D} & =\mathbf{D}^{\mathrm{ep}}+\mathbf{D}^{\mathrm{tr}} \\
\mathbf{D}^{\mathrm{ep}} & =f_{t}^{\alpha^{\prime}} \mathbf{D}_{1}+\left(1-f_{t}^{\alpha^{\prime}}\right) \mathbf{D}_{0} \\
\boldsymbol{\sigma}_{0 t} & =\mathbf{Q}_{t} \cdot \boldsymbol{\sigma}_{0 t-1} \cdot \mathbf{Q}_{t}^{\mathrm{T}}+\mathcal{C}_{0}^{\mathrm{J}, \mathrm{el}}:\left(\Delta t \mathbf{D}_{0}-\Delta \lambda_{0 t} \mathbf{N}_{0 t}\right) \\
\boldsymbol{\sigma}_{1 t} & =\mathbf{Q}_{t} \cdot \boldsymbol{\sigma}_{1 t-1} \cdot \mathbf{Q}_{t}^{\mathrm{T}}+\mathcal{C}_{1}^{\mathrm{J}, \mathrm{el}}:\left(\Delta t \mathbf{D}_{1}-\Delta \lambda_{1 t} \mathbf{N}_{1 t}\right)+\frac{\Delta f^{\alpha^{\prime}}}{f_{t}^{\alpha^{\prime}}}\left(\boldsymbol{\sigma}_{0 t}-\boldsymbol{\sigma}_{1 t}\right) \\
\boldsymbol{\sigma}_{t} & =f_{t}^{\alpha^{\prime}} \boldsymbol{\sigma}_{1 t}+\left(1-f_{t}^{\alpha^{\prime}}\right) \boldsymbol{\sigma}_{0 t} \\
\Delta f^{\alpha^{\prime}} & =f_{t}^{\alpha^{\prime}}\left(\boldsymbol{\sigma}_{0 t}\right)-f_{t-1}^{\alpha^{\prime}} \\
\mathbf{D}^{\mathrm{tr}} & =\frac{\Delta f^{\alpha^{\prime}}}{\Delta t}\left(T_{t} \mathbf{N}_{0 t}+\frac{\delta V}{3} \mathbf{I}\right)
\end{aligned}
$$

are solved at each step, $t$. These equations require a non-linear solution algorithm and in the current study, a combination of the Fixed-Point iteration method, the NewtonRaphson method and a Broyden-type Secant method is utilized (see Appendix B). First, the Fixed Point iterations are used to obtain an initial estimate of the solution. Then, the Newton-Raphson method is utilized for a number of iterations to be followed by the Broyden method until the desired accuracy is reached. After solving the system, all variables are updated for the next time step and the material tangent is computed.

To obtain the explicit expression for the material tangent, the total stress rate must be written as a function of the total deformation rate. Therefore, first, the expression for the total stress state is gathered. The stress rate is only a function of the stress rates of austenite and martensite therefore the following holds:

$$
\stackrel{\nabla}{\boldsymbol{\sigma}}=f^{\alpha^{\prime}} \stackrel{\nabla}{\boldsymbol{\sigma}}_{1}+\left(1-f^{\alpha^{\prime}}\right) \stackrel{\nabla}{\boldsymbol{\sigma}}_{0}+\dot{f}^{\alpha^{\prime}}\left(\boldsymbol{\sigma}_{1}-\boldsymbol{\sigma}_{0}\right)
$$

Substituting the dilution term (Equation (6.54)) and writing Equation (6.56) in terms 
of deformation rates gives:

$$
\begin{aligned}
\stackrel{\nabla}{\boldsymbol{\sigma}} & =f^{\alpha^{\prime}}\left(\mathcal{C}_{1}^{\mathrm{J}, \mathrm{alg}}: \mathbf{D}_{1}+\frac{\dot{f^{\alpha^{\prime}}}}{f^{\alpha^{\prime}}}\left(\boldsymbol{\sigma}_{0}-\boldsymbol{\sigma}_{1}\right)\right)+\left(1-f^{\alpha^{\prime}}\right) \mathcal{C}_{0}^{\mathrm{J}, \mathrm{alg}}: \mathbf{D}_{0}+\dot{f}^{\alpha^{\prime}}\left(\boldsymbol{\sigma}_{1}-\boldsymbol{\sigma}_{0}\right) \\
& =f^{\alpha^{\prime}} \mathcal{C}_{1}^{\mathrm{J}, \mathrm{alg}}: \mathbf{D}_{1}+\left(1-f^{\alpha^{\prime}}\right) \mathcal{C}_{0}^{\mathrm{J}, \mathrm{alg}}: \mathbf{D}_{0}
\end{aligned}
$$

Introducing the strain concentration tensors for each phase (see Chapter 5) gives

$$
\stackrel{\nabla}{\boldsymbol{\sigma}}=f^{\alpha^{\prime}} \mathcal{C}_{1}^{\mathrm{J}, \text { alg }}: \mathcal{A}_{1}: \mathbf{D}^{\mathrm{ep}}+\left(1-f^{\alpha^{\prime}}\right) \mathcal{C}_{0}^{\mathrm{J}, \mathrm{alg}}: \mathcal{A}_{0}: \mathbf{D}^{\mathrm{ep}}
$$

which can be written as:

$$
\stackrel{\nabla}{\boldsymbol{\sigma}}=\left(f^{\alpha^{\prime}} \mathcal{C}_{1}^{\mathrm{J}, \text { alg }}: \mathcal{A}_{1}+\left(1-f^{\alpha^{\prime}}\right) \mathcal{C}_{0}^{\mathrm{J}, \text { alg }}: \mathcal{A}_{0}\right):\left(\mathbf{D}-\mathbf{D}^{\mathrm{tr}}\right) .
$$

Substituting the transformation strain obtained by Equation (6.53) gives:

$$
\mathcal{C}^{\mathrm{J}}=\left(f^{\alpha^{\prime}} \mathcal{C}_{1}^{\mathrm{J}, \mathrm{alg}}: \mathcal{A}_{1}+\left(1-f^{\alpha^{\prime}}\right) \mathcal{C}_{0}^{\mathrm{J}, \mathrm{alg}}: \mathcal{A}_{0}\right):\left(\mathcal{I}-\frac{\mathbf{T} \otimes \frac{\mathrm{d} f^{\alpha^{\prime}}}{\mathrm{d} \boldsymbol{\sigma}_{0}}: \mathcal{C}_{0}^{\mathrm{J}, \mathrm{alg}}: \mathcal{A}_{0}}{1+\frac{\mathrm{d} f^{\alpha^{\prime}}}{\mathrm{d} \boldsymbol{\sigma}_{0}}: \mathcal{C}_{0}^{\mathrm{J}, \mathrm{alg}}: \mathcal{A}_{0}: \mathbf{T}}\right)
$$

The material tangent computed by Equation (6.60) is a linearization of the stress rate with respect to the deformation rate. However, it is not the exact linearization for the presented algorithm. The strain concentration terms that appear in the homogenization algorithm, $\mathcal{A}$, and the transformation deformation, $\mathbf{T}$, are linearized only to a first order and the variation of these terms with respect to the incremental deformation are neglected. The material tangent is used for the prediction of the next iterative solution in the Finite Element solution procedure and does not have an effect on the accuracy of the solution. A consistent linearization results in a quadratic convergence of the Newton-Raphson scheme. The derivation above, although not exact, is sufficient to achieve stable convergence behavior. It is important to note that the derived material tangent has minor symmetries but not the major one.

\subsection{Results}

In this section, the results of the constitutive model on the integration point level are presented. To be able to compare with the experiments presented in Chapter 3, an equivalent deformation has been generated in 3-D as a collection of deformation gradients. These were supplied as input to the stress update algorithm and the resulting martensite fraction and stress were computed. To simulate the plane-stress condition, the deformation in the thickness direction was solved at each step in an outer loop. In all tests, except the ones at different temperatures, all parameters related to transformation were kept constant. For the tests at different temperatures, only the critical energy barrier and the hardening behavior of the austenite were modified. The homogenization algorithm used in the simulations was the Lielens model with the proposed interpolation function $(\mathrm{PG})$. 
The parameters used in the transformation function (Equation (4.25)) were:

$$
\begin{aligned}
\Delta G^{\mathrm{cr}} & =58 \mathrm{MPa}, \\
m & =27, \\
r & =4, \\
\Delta G^{\mathrm{off}} & =6 .
\end{aligned}
$$

\section{Stress state}

In Figure 6.2, model results in a simple shear simulation are compared to the experimental results in terms of martensite fraction and shear stress. As a starting point, the results show that the model is able to capture the characteristic sigmoidshape transformation behavior of the material. Quantitatively a good agreement is observed with the experiments in terms of predicted martensite fraction. The shear stress-equivalent strain curve reveals that the relaxation effect of transformation plasticity is well-captured by the model, which shows itself as a plateau after the initial yield point. Following the relaxation, as the martensite fraction increases, the material behavior gradually changes towards that of martensite. This is observed as a steep hardening in the stress response. Finally, this hardening is followed by a relatively soft behavior which is a consequence of the yielding of martensite particles.
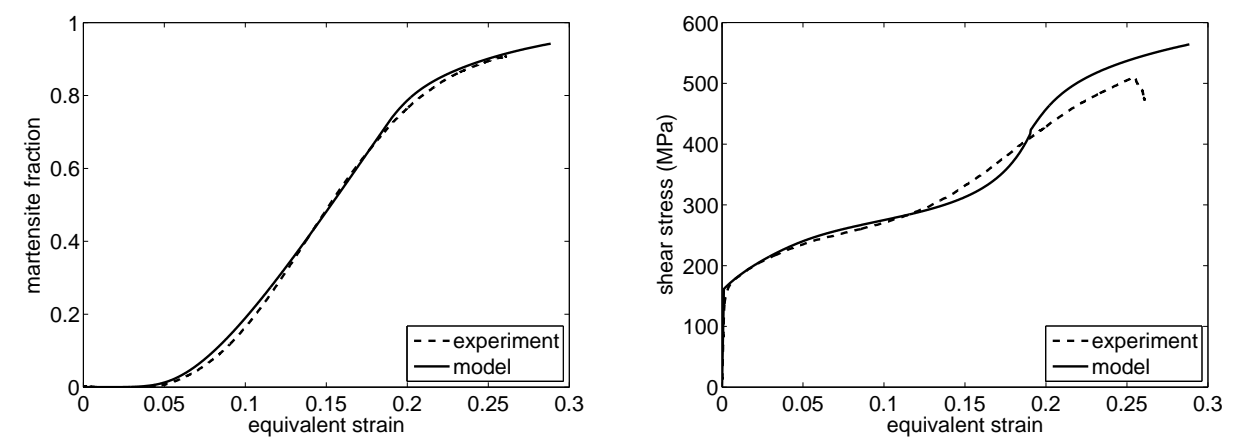

Figure 6.2: Calculated martensite fraction (left) and stress (right) under a simple shear deformation and comparison with experimental results.

Figure 6.3 shows the martensite fraction and the tensile stress developed in the material under plane-strain tension deformation. It is clear that the model has reproduced, successfully, the stress state dependency of the transformation. The calculated martensite fraction is observed to agree very well with the experimental results. The experimental results show that there is some anisotropy in the plastic behavior of austenite. This can be observed in the difference between the calculated yield stress, which is based on an isotropic yield surface, and the experimentally observed yield stress. The transformation plasticity and the hardening behavior is captured by the model successfully. 

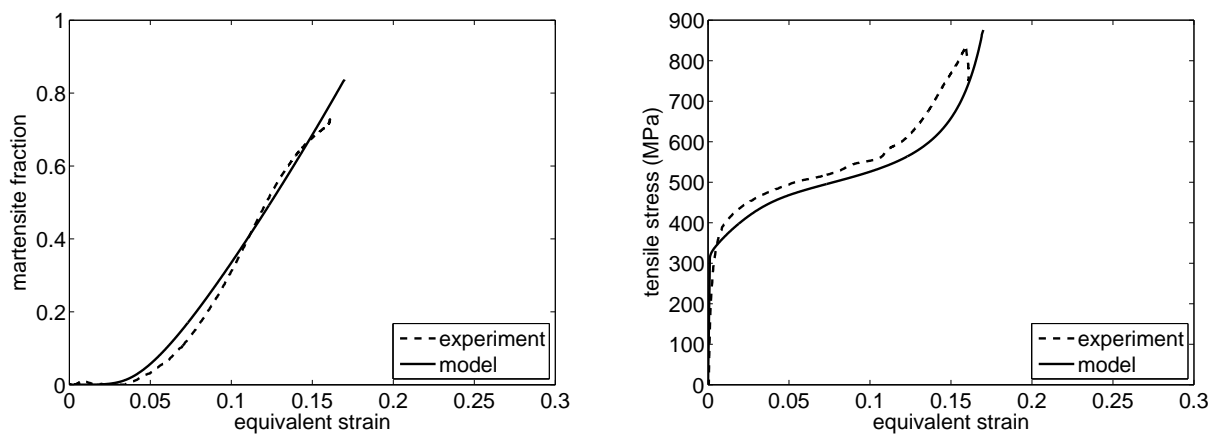

Figure 6.3: Calculated martensite fraction (left) and stress (right) under a plane strain tension deformation and comparison with experimental results.

Finally, Figure 6.4 shows the results of the model under a combined plane-strain, simple shear test with a 45 degrees deformation angle. The results are observed to be in good agreement with the experiments in terms of martensite fraction as well as the shear and tensile stresses.
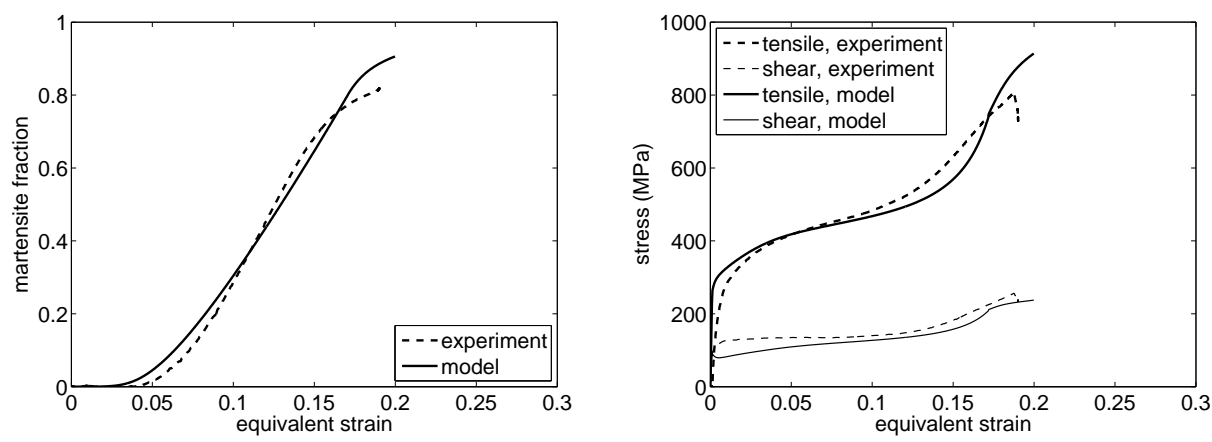

Figure 6.4: Calculated martensite fraction (left) and stress (right) under a combination of simple shear and plane strain tension deformations $(\alpha=45)$ and comparison with experimental results.

In these set of results, one common observation is the stiff response calculated by the model compared to the experiments. One of the reasons for this behavior can be due to the uncertainty in the stress-strain relation defined for the martensite phase. As mentioned before, it is very challenging to determine the exact mechanical behavior of martensite in this material. One other place to look for the source of the error is the homogenization algorithm. There are a number of assumptions made which can influence the behavior of the composite. For instance, as mentioned in Chapter 5, the definition of an inclusion and a matrix for moderate volume fractions is ambiguous and the interconnectivity of the phases might influence the results.

Another observation is that the predicted saturation value for the martensite fraction 
is higher than that observed experimentally. In the model a total isotropy in the transformation behavior is assumed, in other words the effect of a crystallographic texture is not incorporated. If the material has texture, it can play a role on the saturation behavior of the martensite fraction. On the experimental side, it has been discussed in Chapter 3 that due to the setup, errors are introduced in the measured martensite fraction at high volume fractions. Therefore, the discrepancy can also be due to the accuracy of the measurements.

Considering the factors mentioned above, it can be concluded that the model is successful in predicting the overall mechanical behavior of the material as well as the stress-state dependency of the mechanically induced martensitic transformation.

\section{Temperature}

It is known that the transformation behavior is strongly temperature dependent. In the model, this dependency is not handled explicitly but incorporated through the transformation parameters. It has been discussed in Chapter 4 that the critical energy barrier is temperature dependent since:

$$
\Delta G^{\mathrm{cr}}=\left.\Delta G^{\gamma \rightarrow \alpha^{\prime}}\right|_{T}-\left.\Delta G^{\gamma \rightarrow \alpha^{\prime}}\right|_{M_{s}}
$$

and the free energy difference of austenite and martensite is a function of temperature. The free energy difference can be assumed to be linear in temperature and can be described as:

$$
\left.\Delta G^{\gamma \rightarrow \alpha^{\prime}}\right|_{T}=B\left(T-T_{0}\right)
$$

where $B$ is the proportionality coefficient and $T_{0}$ is the equilibrium temperature. Since $\left.\Delta G^{\gamma \rightarrow \alpha^{\prime}}\right|_{M_{s}}$ is constant, the change in the energy barrier can be represented by a linear relation with respect to temperature:

$$
\Delta G^{\mathrm{cr}}=\left.\Delta G^{\mathrm{cr}}\right|_{\mathrm{RT}}+B \Delta T
$$

where, $\Delta T=T-T_{\mathrm{RT}}$.

The proportionality constant can be approximated using the results of the prestrain experiments. In the tests, the critical stress at which the transformation starts can be observed at two different temperatures: RT and $120^{\circ} \mathrm{C}$. Using these results $B$ is found to be approximately $0.2 \mathrm{MPa} / \mathrm{K}$.

Additionally, the flow stress of austenite is known to decrease with increasing temperature. Following a similar linear approximation, the flow stress at a given temperature can be found by:

$$
\left.\sigma_{0}^{f}\right|_{T}=\left.\sigma_{0}^{f}\right|_{\mathrm{RT}}(1-D \Delta T)
$$

where $D$ is estimated using the experimental results as $0.0018 \mathrm{~K}^{-1}$.

Using these results, the temperature dependency of the transformation can be incorporated in the model. The simulated transformation behavior during a simple shear test at different temperatures is presented in Figure 6.5. 

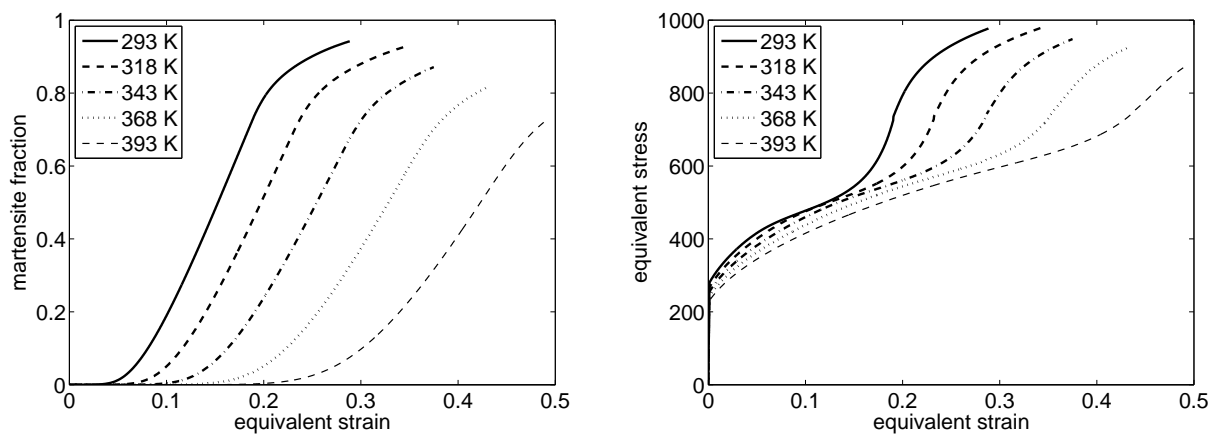

Figure 6.5: Predicted martensite fraction (left) and equivalent stress (right) during a simple shear deformation at different temperatures.

The results follow the expected trend where transformation gets slower as the temperature increases. At $120{ }^{\circ} \mathrm{C}(393 \mathrm{~K})$, for a plastic deformation of $25 \%$, only a few percent of martensite forms, which agrees well with experimental observation in the prestrain tests.

\subsection{Summary and conclusions}

The FE equations in an Updated Lagrangian setting were presented to demonstrate the requirements from the constitutive model. It was shown that as a start, the model has to provide the new stress state of an integration point for a given deformation to check if equilibrium is satisfied at the structure level. Additionally, the model has to supply the material tangent using the state variables at the end of the time step, to be used in building the stiffness matrix for the next iteration.

Constructed constitutive model consists of two interwoven algorithms: homogenization and transformation. The total deformation is split into transformation and elasticplastic parts, additively. The elastic-plastic part is passed to the homogenization algorithm, where it is further partitioned into the deformation in austenite and martensite phases. The stress-update in each phase is carried out using conventional procedures. For simplicity, both phases were assumed to follow the J2 flow plasticity with isotropic hardening which enables utilization of the radial-return mapping algorithm.

The resulting rate equations were solved using a Backward-Euler approach and the algorithm was linearized to get the tangent operator. The linearization was carried out analytically, but not exactly. Although the tangent operators gathered for the individual phase responses are exact, the homogenization and the transformation equations were derived in a continuum fashion.

The results of the stress-update were presented on test problems which demonstrate the effects of stress-state and temperature on the transformation behavior. The 
model was found to capture the characteristics of the transformation using only a few parameters which could be determined by a single tensile test. The parameters to be determined were: the critical energy barrier, which was calculated using the observed critical stress, and the fitting parameters in the transformation function, $m$, $r$ and $\Delta G^{\text {off }}$.

The effect of temperature was incorporated in the model indirectly via the dependence of the critical energy barrier and the flow stress of austenite on temperature. To observe the temperature dependency of the model, two tensile tests were performed at different temperatures at which the critical stress and the yield stress of austenite were determined. Assuming a linear relation for these parameters, a set of simulations were performed at different temperatures. The model results were found to agree qualitatively with experimental observations. 


\section{Application}

The material model has been implemented in the implicit Finite Element software DiekA for conducting simulations at the structure level. For validation of the model, simulation of bending of a plate was considered. This example demonstrates the stress-state dependency of the transformation which results in an asymmetric transformation profile through the thickness of the plate.

\subsection{Bending simulation}

For simulation of plate bending, an idealized setup as shown in Figure 7.1 was utilized. The problem has a plane-strain nature on the $x-z$ plane due to the relative dimensions of the plate: $\mathrm{l}=10 \mathrm{~mm}, \mathrm{t}=2.1 \mathrm{~mm}, \mathrm{w}=30 \mathrm{~mm}$. These dimensions were chosen to be able to compare the results with the experiments reported in [49].

In the simulation, a mesh with $20 \times 6 \times 1,8$-node hexahedral (brick) elements was created along the $x, z$ and $y$ axis, respectively. The elements have been improved using the ' $\overline{\mathrm{B}}$ ' method [62] to avoid volumetric locking.

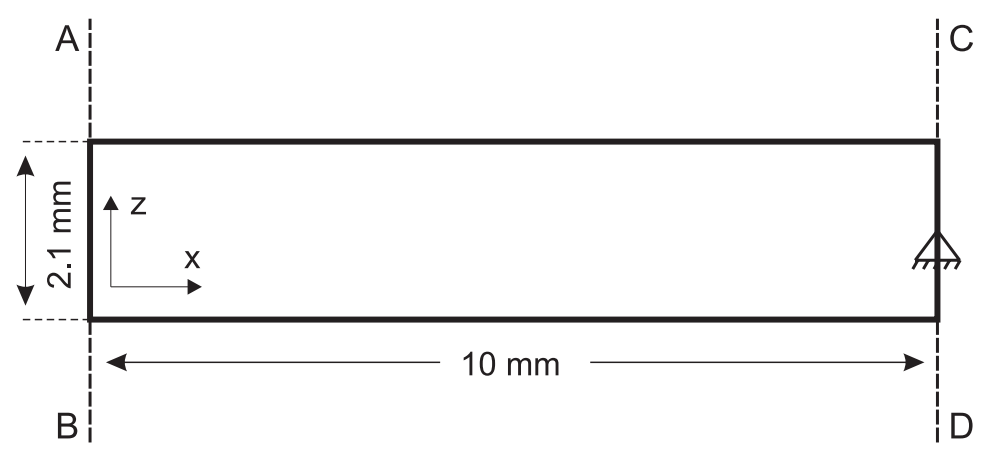

Figure 7.1: Bending simulation setup. 
To establish the plane-strain condition, displacement of all nodes in the $y$ direction was suppressed. Bending was applied by incrementally rotating the tip of the plate in the $x-z$ plane where the displacement of the mid-point of the tip was suppressed. The $x$ and $z$ displacements of all the nodes on the line C-D were coupled to keep the edge on a straight line. The $x$ displacement of the nodes on the line A-B were connected to keep the edge always vertical during the simulation. As this edge was free to move horizontally and vertically, the deformation satisfied the pure bending condition.

During the simulation, in the loading stage, a total clockwise rotation of 120 degrees was prescribed in 120 increments. During unloading, increments of a rotation of 0.1 degrees in the counter-clockwise direction were prescribed.

Prescribing the displacements at the edge of the plate and the application of a line constraint causes a local disturbance of the solution in the vicinity of the boundary. On the other hand, the affected zone is small and does not influence the results observed far from that boundary.

The austenite and martensite material properties are selected as:

Table 7.1: Material parameters used for the austenite and martensite phases.

\begin{tabular}{rrccccc}
\hline Phase & $E(\mathrm{MPa})$ & $\nu$ & $\sigma^{\mathrm{y}}(\mathrm{MPa})$ & $\varepsilon^{0}$ & $K$ & $m$ \\
\hline Austenite & 210000 & 0.3 & 280 & 0.01 & 1000 & 0.51 \\
Martensite & 210000 & 0.3 & 800 & 0.001 & 1000 & 0.07 \\
\hline
\end{tabular}

The parameters used for transformation were (see Equation (4.25)):

$$
\begin{aligned}
\Delta G^{\mathrm{cr}} & =58 \mathrm{MPa}, \\
m & =27, \\
r & =4, \\
\Delta G^{\mathrm{off}} & =6 .
\end{aligned}
$$

\section{$7.2 \quad$ Results}

Figures 7.2 and 7.3 show the distribution of the martensite phase and equivalent (Von Mises) stress during bending. It is clearly observed that the transformation behavior is asymmetric. Transformation initiates first at the tension side of the plate and proceeds faster on this side. This is an expected result since the transformation model is stress-state dependent. The transformation surface (Figure 4.2) that has been established in Chapter 4 reflects this dependency and shows that transformation starts earlier under tension than compression.

The asymmetry is more clearly visualized in Figure 7.4 where the volume fraction of martensite through the thickness of the plate for different bending angles is plotted. 
$\alpha=0$
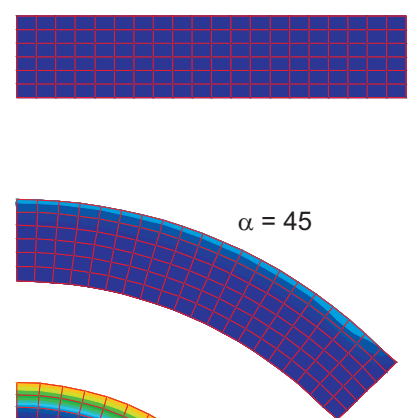

$\alpha=90$

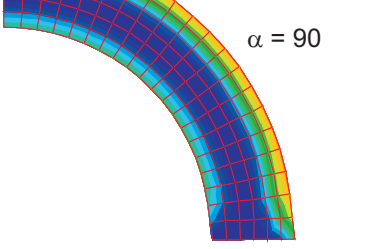

$\alpha=15$

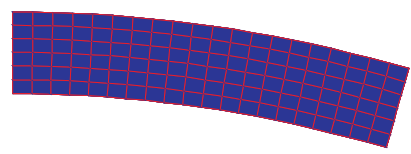

$\alpha=60$

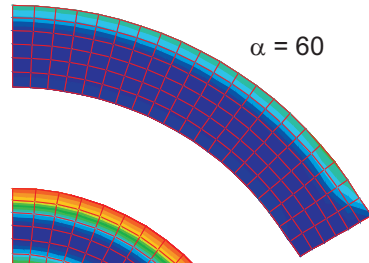

$\alpha=105$
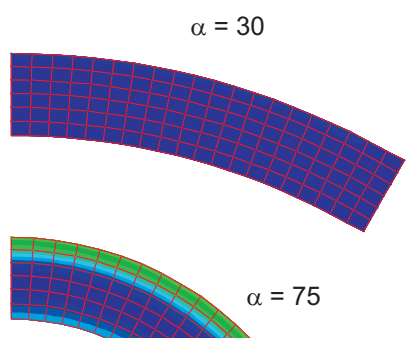

$\alpha=75$

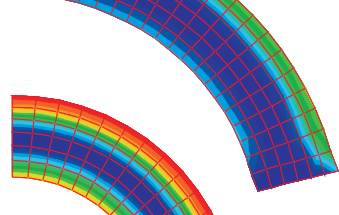

$\alpha=120$
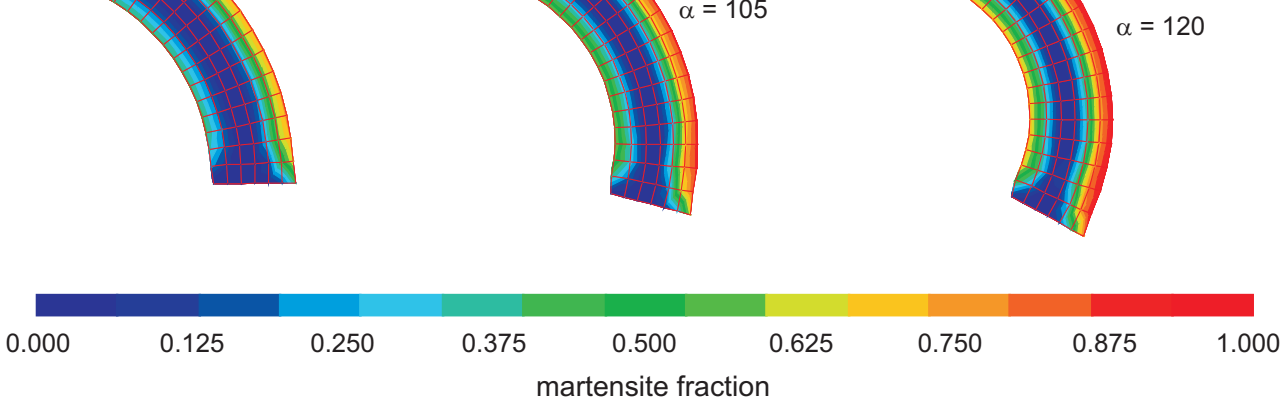

Figure 7.2: Martensite fraction over the part during pure bending ( $\alpha$ denotes tip rotation).

The corresponding bending stress profile through the thickness of the material is shown in Figure 7.5.

In [49], the distribution of martensite fraction through the thickness of a plate of same dimensions have been reported. Figure 7.6 shows comparison of the model results with the experimental results. It is seen that the model has captured the asymmetry as well as the shift of the neutral line satisfactorily. However in the experimental results, transformation is observed to start earlier compared to the model and proceeds faster close to the top and bottom surfaces. This discrepancy can be attributed to the differences in the material. It is known that the stability of austenite in this material is very sensitive to the chemical composition and prior processing conditions. The material data used in the model has been gathered by experiments done on a thinner sample $(\mathrm{t}=0.5 \mathrm{~mm})$.

The bending moment vs. curvature diagram gathered from the simulation is shown in Figure 7.7. The experimentally obtained moment-curvature diagram of this material reveals an interesting phenomenon which shows itself as a non-linear unloading behavior (schematically shown in Figure 7.7). In the report, one of the possible reasons of this behavior is proposed to be the continuation of transformation during unloading. In the simulations, upon loading any change in the martensite fraction is not observed. 

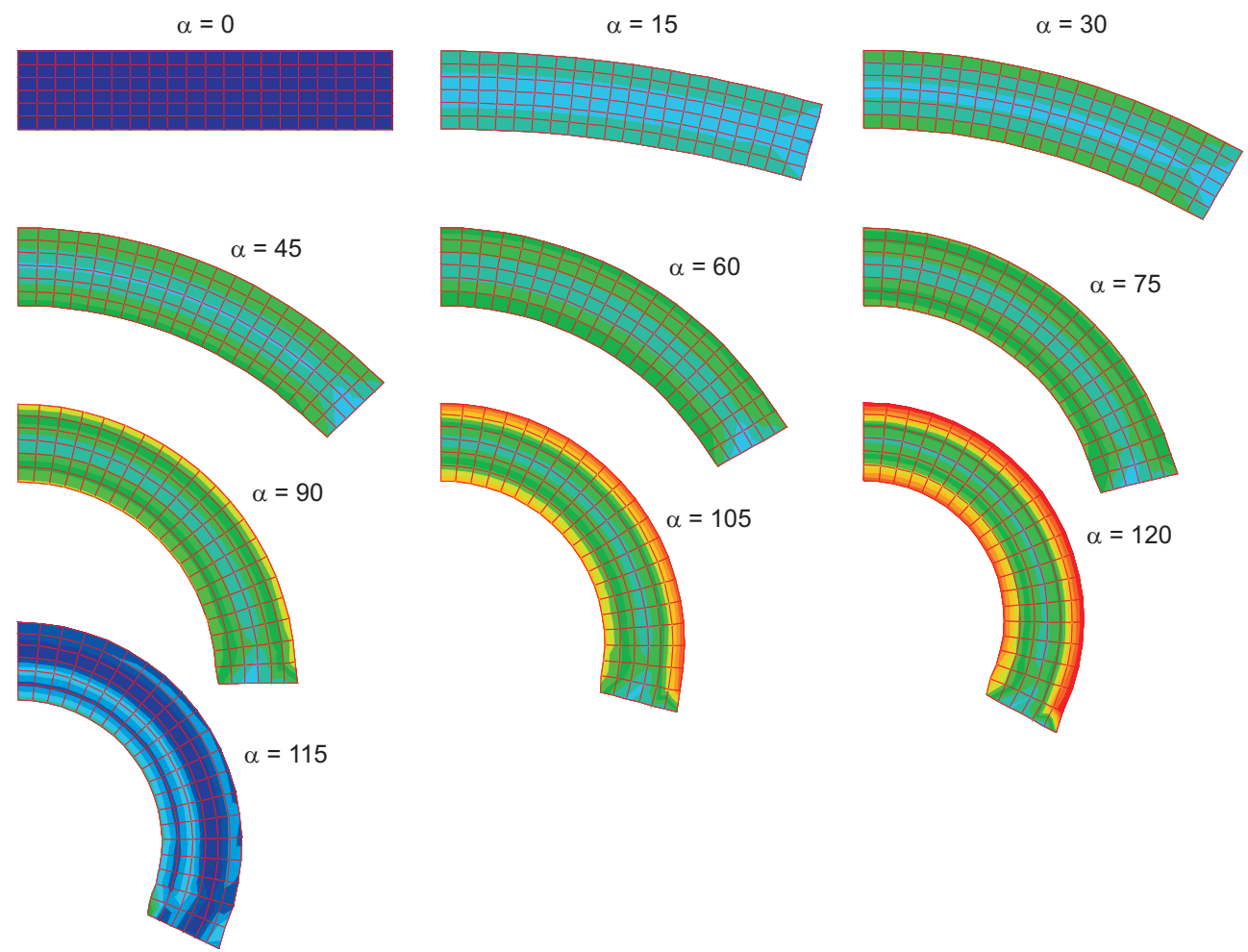

$\begin{array}{ccccccccc}0.000 & 0.125 & 0.250 & 0.375 & 0.500 & 0.625 & 0.750 & 0.875 & 1.000 \\ & & \text { equivalent stress }(\mathrm{GPa}) & \end{array}$

Figure 7.3: Equivalent stress over the part during pure bending and after unloading ( $\alpha$ denotes tip rotation).

In the constitutive model, transformation is described by a single scalar: martensite fraction. The current martensite fraction in an integration point in turn dictates the current energy barrier at that point since more transformation will only take place when driving force exceeds the value that could transform the current amount of martensite. Hence, in the model there is no explicit treatment of the texture in the material. This is expected to result in a discrepancy of the results with the experiments since in [31] it is reported that the material actually has a pronounced texture.

Additionally, although the evolution of the martensite fraction is stress-state dependent, there is no history dependent parameters in the model to account for the anisotropy formation due to transformation. When the material is being loaded proportionally, the grains that are favorable in the stress direction transform gradually according to their relative orientation. If a strain-path change occurs, then it is 


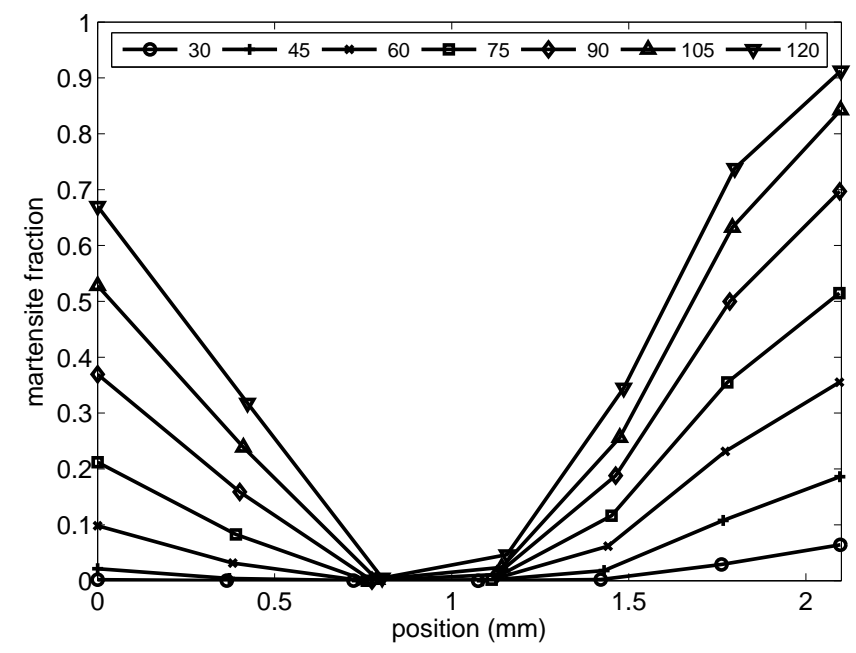

Figure 7.4: Martensite distribution over the thickness at various bending levels. Legend denotes the tip rotation (in degrees).

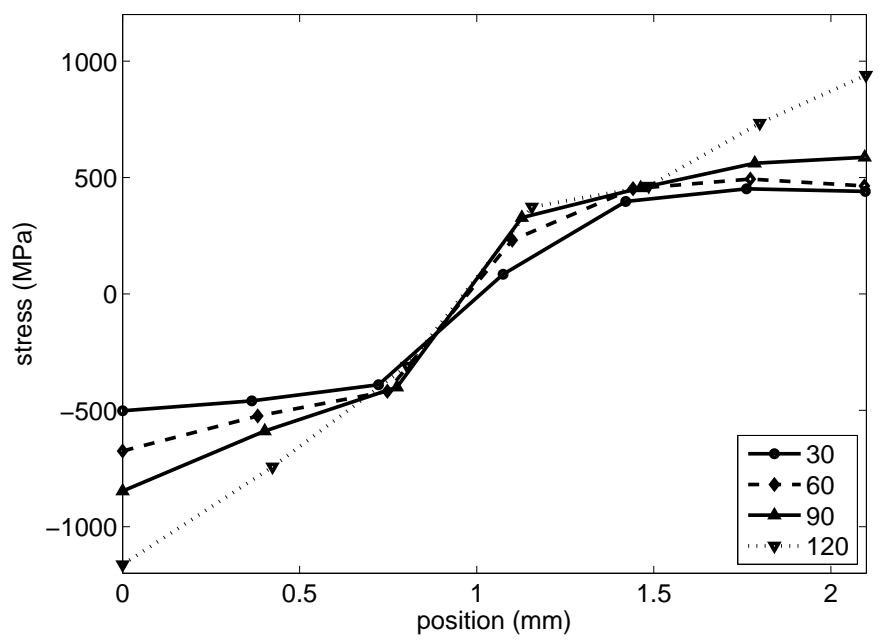

Figure 7.5: Stress distribution over the thickness at various bending levels. Legend denotes the tip rotation (in degrees).

expected that the grains that are the most favorable in the new stress direction will transform. This implies that the energy barrier in the new direction will be the initial critical energy barrier assigned as a parameter. This can cause a difference, that would show itself as an underestimation of the transformation, with the experiments 


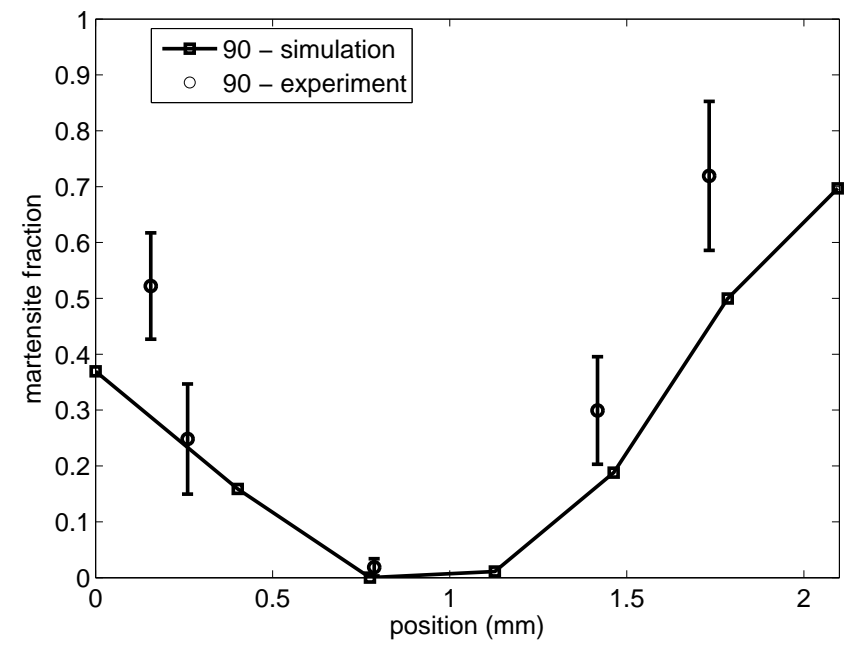

Figure 7.6: Martensite distribution over the thickness at 90 degrees of tip rotation and comparison with experimental data [49].

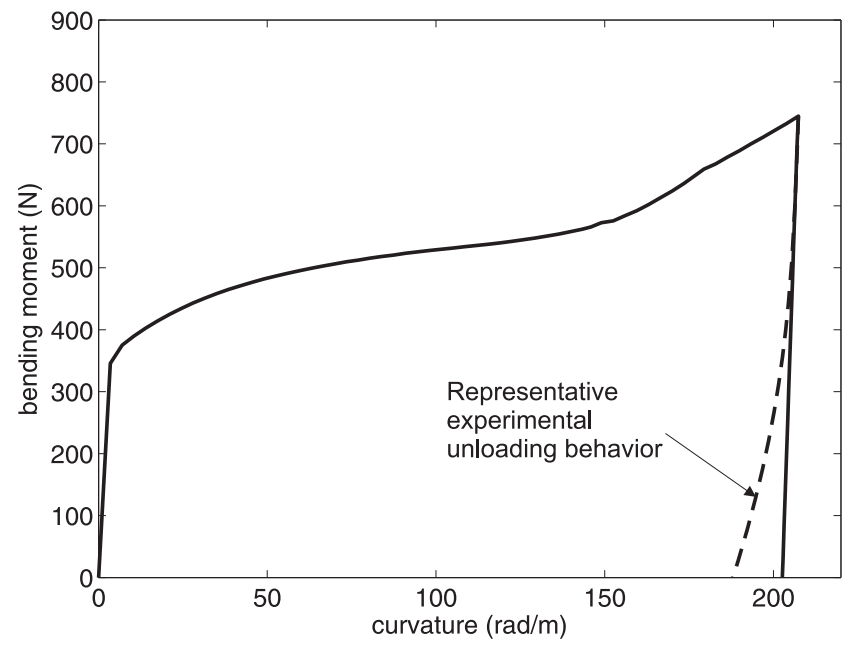

Figure 7.7: Calculated bending moment vs. curvature diagram.

for instance during unloading. 


\subsection{Summary and conclusions}

The developed constitutive model was implemented in the implicit FE code DiekA to be tested at the structure level. Simulation of a plate under pure pending was chosen as a demonstrative example for validation. The results were found to be in good qualitative agreement with experiments. The asymmetric transformation phenomenon was captured satisfactorily with the model.

Experimentally observed non-linear unloading behavior was not reproduced by the model. The non-linearity might be attributed to transformation during unloading which can be caused by the change of stress state during the process. The developed transformation model is based on an isotropic transformation criterion meaning that the influence of non-proportional loading cannot be captured. 



\section{Conclusions and Recommendations}

Accurate simulations of forming processes using the finite element method require reliable constitutive models to describe the mechanical behavior of materials. For the case of metastable austenitic stainless steels, the relation between stress and strain is a very strong function of the phase transformation that takes place during the deformation. In the present work a new, physically based constitutive model is presented that is shown to capture the characteristics of the mechanical behavior of these materials. In the thesis, the experimental and theoretical studies that were performed to obtain the model were discussed. In this chapter, the conclusions drawn from these studies and recommendations for further research are presented.

\section{Experiments}

In the literature, two competing approaches were found to describe the mechanically induced martensitic transformation phenomenon: micromechanical models which are mostly focused on the action of applied stress and continuum models which are built on strain-induced transformation theory.

It was demonstrated that the stress-state has a considerable influence on the transformation kinetics. This observation can be put into the perspective of straininduced transformation theory only indirectly, via the relation between the probability of nucleation and the stress-state. On the other hand, the effect of the stress-state is inherent in the stress-driven approach. Additionally, it was found that the results of prestrain tests cannot be explained consistently by the strain-induced transformation theory.

These results suggest that a stress-driven approach can be used to describe the physics behind the transformation just as well as the strain-induced transformation theory. However, it is recommended that more experimental studies be carried out in order to arrive at a full understanding of the effect of plastic strain on the transformation. 
Although there are two different theories to explain the transformation, they need not be excluding each other.

\section{Transformation model}

The stress-driven approach is generally used in micromechanical studies assuming that transformation is related to the action of the resolved driving force on each potential martensitic variant. To be able to test this theory, a simple model in mesoscale was developed. A good qualitative correspondence with the experiments was achieved using a single physical parameter: the critical energy barrier. According to this model the macroscopically observed transformation behavior is a consequence of the distribution of the driving force over the material.

A transformation criterion was developed and represented as a transformation surface in the principal stress space. Having a very good agreement with the experiments, the results prove the validity of the inherent stress-state dependency of the stress-driven approach.

In all the mechanical tests a similar transformation behavior has been observed with respect to the applied maximum mechanical driving force. Based on this observation, a transformation model was proposed that described the evolution of the martensite fraction with respect to the applied stress. The parameters of this model can be determined by a single tensile test.

In the developed model, it is the increase in the resolved stress that causes an increase in the martensite volume fraction. In the plastic regime, the increase in stress can only be achieved by strain hardening which makes the transformation, indirectly, a function of equivalent plastic strain.

The developed transformation model has not yet been tested on other grades of austenitic stainless steels. It is recommended that similar mechanical tests be performed on these materials.

\section{Homogenization}

To model the stress-strain behavior of the material, a Mean-Field homogenization model was developed. Different homogenization schemes were investigated and the most reliable one theoretically was found to be the Self-Consistent model. This model is not limited to low volume fraction of inclusions as opposed to other models found in the literature. An alternative model was proposed which is computationally less expensive and in most cases comparable to the Self-Consistent model in terms of accuracy.

It is known that the Mean-Field method has shortcomings, mainly because the local fields around the inhomogeneities are not taken into account and the validity of Eshelby's results are questionable at intermediate volume fractions. Therefore, it is recommended that further research on homogenization be conducted in order to achieve higher accuracy. This can be accomplished, for instance, by constructing Direct Finite Element simulations for comparison on representative problems. 


\section{Constitutive model}

The transformation and the homogenization algorithms were merged to form the constitutive model. In addition to these, the effects of transformation on the partitioning of stress between the phases were considered and implemented. To observe clearly the behavior of the stress-driven transformation model and the associated change in mechanical behavior, the material models for the individual phases were kept simple.

A good agreement with the biaxial test results was obtained which supports the theory that the transformation can be explained as a stress-driven process. The effect of temperature was analyzed by assuming simple linear relations for the critical energy barrier and the flow stress of austenite with temperature and qualitatively good results were obtained.

It is recommended that more tests be carried out at different temperatures to check the results also quantitatively. Additionally, the proposed stress partitioning theories can be verified by performing Direct Finite Element simulations on representative problems.

For further improvement of the model proposed in this work, the material models used for individual phases can be refined to reflect more accurately their mechanical behavior. This would require performing a number of carefully designed mechanical tests. It has been observed that the material used in this study has an anisotropic yield behavior. This anisotropy in the material can be determined experimentally and the constitutive formulations can be adapted according to the findings.

It is also known that the material has a crystallographic texture. The effects of the texture on the transformation can be formulated by making use of the developed mesoscale (multi-grain) model. Furthermore, this model can be used to investigate the consequences of a strain-path change during deformation. Although the continuum model is applicable in this stage under total isotropy and monotonic loading conditions, it can be further improved by incorporating these two effects. 



\section{Expressions for the Eshelby tensor}

Eshelby tensor is one of the results of Eshelby's solution of the inclusion problem [20]. In this problem, an inclusion (i.e. an arbitrary section in a continuum) undergoes a stress-free and prescribed eigenstrain. The problem is then to find the equilibrated strain in the inclusion. Eshelby showed that if the matrix is isotropic and inclusion is ellipsoid in shape, then the resulting strain in the inclusion is uniform. For this case, Eshelby has analytically derived a $4^{\text {th }}$ order tensor which operates on the eigenstrain tensor and returns the strain in the inclusion.

Eshelby tensor has minor symmetries but not the major one. Furthermore, in case of an isotropic matrix and an ellipsoidal inclusion, it is only a function of the shape of the inclusion and the Poisson's ratio of the material. In the book by Mura [61], simple expressions for the tensor for basic shapes of inclusions can be found.

In this study, due to the random alignment of inhomogeneities, Eshelby tensor is only computed for the case of spherical inclusions. In this case, the Eshelby tensor is isotropic and is given by:

$$
\mathcal{E}=\frac{1+\nu}{9(1-\nu)} \mathbf{I} \otimes \mathbf{I}+\frac{2(4-5 \nu)}{15(1-\nu)}\left(\mathcal{I}-\frac{1}{3} \mathbf{I} \otimes \mathbf{I}\right)
$$

where, $\nu$ is the Poisson's ratio. In the plastic regime:

$$
\mathcal{E}=\frac{3 \alpha}{3 \alpha+4 \beta} \mathbf{I} \otimes \mathbf{I}+\frac{6}{5} \frac{\alpha+2 \beta}{3 \alpha+4 \beta}\left(\mathcal{I}-\frac{1}{3} \mathbf{I} \otimes \mathbf{I}\right)
$$

where, $\alpha$ and $\beta$ are the volumetric and deviatoric coefficients of a $4^{\text {th }}$ order isotropic material tensor.

In the general spheroidal case, the tensor is not isotropic due to the apparent anisotropy introduced by the geometry of the inclusion. The spheroid can be expressed by an aspect ratio $a$, and the alignment direction. The latter will be taken as the 
direction 1 since any other direction can be obtained by consistently rotating the tensor. In this case the non-zero components of the tensor are given as:

$$
\begin{aligned}
E_{1111} & =\frac{1}{2(1-\nu)}\left[2(1-\nu)(1-I)+I-a^{2} \frac{3 I-2}{a^{2}-1}\right], \\
E_{2222}=E_{3333} & =\frac{1}{4(1-\nu)}\left[2(2-\nu) I-\frac{1}{2}-\left(a^{2}-\frac{1}{4}\right) \frac{3 I-2}{a^{2}-1}\right], \\
E_{1122}=E_{1133} & =\frac{1}{4(1-\nu)}\left[4 \nu(1-I)-I+a^{2} \frac{3 I-2}{a^{2}-1}\right], \\
E_{2233}=E_{3322} & =\frac{1}{4(1-\nu)}\left[-(1-2 \nu) I+\frac{1}{2}-\frac{1}{4} \frac{3 I-2}{a^{2}-1}\right], \\
E_{2211}=E_{3311} & =\frac{1}{4(1-\nu)}\left[-(1-2 \nu) I+a^{2} \frac{3 I-2}{a^{2}-1}\right], \\
E_{1212}=E_{1313} & =\frac{1}{4(1-\nu)}\left[(1-\nu)(2-I)-I+a^{2} \frac{3 I-2}{a^{2}-1}\right], \\
E_{2323} & =\frac{1}{8(1-\nu)}\left[(5+2 \nu) I-4 \nu-\frac{1}{2}+\left(\frac{1}{4}-2 a^{2}\right) \frac{3 I-2}{a^{2}-1}\right], \\
E_{i j k l} & =E_{j i k l}=E_{i j l k},
\end{aligned}
$$

where,

$$
\begin{aligned}
& I=\frac{a}{\left(a^{2}-1\right)^{3 / 2}}\left[a\left(a^{2}-1\right)^{1 / 2}-\cos ^{-1} a\right] \quad \text { when } a<1, \\
& I=\frac{a}{\left(1-a^{2}\right)^{3 / 2}}\left[\cosh ^{-1} a-a\left(1-a^{2}\right)^{1 / 2}\right] \quad \text { when } a>1 .
\end{aligned}
$$




\section{Fixed Point Iteration and Broyden methods}

For solving a non-linear system of equations one of the mostly used methods is the Newton's method due to the fact that it is robust and converges quadratically [30] . However, this method requires information on the Jacobian of the system, which is not always analytically obtainable. To find the Jacobian approximately, a numerical differentiation can be performed by the Finite Difference method however it is clear that in a system with a large number of equations this would be very time consuming. Fortunately, there are alternative methods that do not require the calculation of the Jacobian by differentiation.

One of these methods is the Fixed Point Iteration method which does not use the Jacobian in searching for a solution. This method has a simple algorithm which only requires the equations to be rearranged to give:

$$
\mathbf{x}_{k+1}=\mathbf{g}\left(\mathbf{x}_{k}\right)
$$

where $k$ is the iteration counter and $\mathbf{g}$ is the system of equations.

This scheme converges to the solution if the condition:

$$
|\mathbf{J}(\mathbf{x})|<1
$$

is satisfied and diverges if not; where $|\mathbf{J}(\mathbf{x})|$ is the norm of the Jacobian calculated at the solution. If the LHS equals 0 then a quadratic convergence rate is obtained. It is not always necessary to check the convergence behavior with this criterion since the implementation is trivial.

In cases where the Fixed Point Iteration method does not converge or is very slow, a Secant Update method can be used. In a one-dimensional setting, the secant method is a good alternative for the Newton's method since it does not require an explicit derivative calculation and estimates the derivative by the change of the function in successive iterates. In solving a system of equations, a similar approach can be 
followed by approximating the Jacobian matrix using the function values at successive iterates.

One of the simplest and most effective Secant Update methods is the Broyden's method [30] in which, the estimate to the Jacobian is given by:

$$
\mathbf{B}_{k+1}=\mathbf{B}_{k}+\frac{\left[\left(\mathbf{y}_{k}-\mathbf{B}_{k} \Delta \mathbf{x}_{k}\right) \Delta \mathbf{x}_{k}^{\mathrm{T}}\right]}{\left(\Delta \mathbf{x}_{k}^{\mathrm{T}} \Delta \mathbf{x}_{k}\right)}
$$

where

$$
\mathbf{y}_{k}=\mathbf{g}\left(\mathbf{x}_{k+1}\right)-\mathbf{g}\left(\mathbf{x}_{k}\right)
$$

Having estimated the Jacobian, the next incremental solution is found as in the Newton's method by solving:

$$
\mathbf{B}_{k} \Delta \mathbf{x}_{k}=-\mathbf{g}\left(\mathbf{x}_{k}\right)
$$




\section{Calibration of the magnetic sensor}

In the biaxial experiments a magnetic sensor is utilized for measuring the volume fraction of martensite in the material. The sensor consists of a coil and operates on the principle that martensite is ferromagnetic whereas austenite is paramagnetic. The output signal is a measure of the impedance of the coil which is sensitive to the magnetic properties of the material that lies in the magnetic field created by the coil. When a ferromagnetic material is in contact with the sensor, the impedance changes, resulting in a change in the output voltage. To convert the measured voltage data into martensite fraction a calibration procedure is required. In the experiments reported in Chapter 3 the following procedures are implemented.

In the experimental setup the sensor is fixed on the lower clamp and stays in contact with the sample throughout the test. The clamps are made of tool steel which is a ferromagnetic material hence, the sensor reading is influenced by the presence of the clamps. During a shear test, the distance of the clamps to the sensor remains constant thus, removing the disturbance is trivial. However, during a test that involves plane strain tension deformation, the distance of the upper clamp to the sensor changes in time. To account for this effect, the upper clamp position is recorded during the test and the disturbance is removed accordingly.

An important phenomenon that has to be taken into account during calibration is magnetostriction, which can be briefly summarized as the change of the magnetic permeability of a material under stress. This effect comes into picture due to the increase in the stress resolved in martensite during the tests. To analyze the magnitude of the disturbance, a series of tests have been performed. The sample is first deformed to have a martensitic volume fraction of around 0.5 and then systematically loaded in shear and tension while the changes in the sensor output are recorded. Figure C.1 shows the results of this procedure. These data are then used to eliminate the effect of stress on the recorded voltage.

Having obtained a clean signal, the actual correlation of the voltage to martensite 


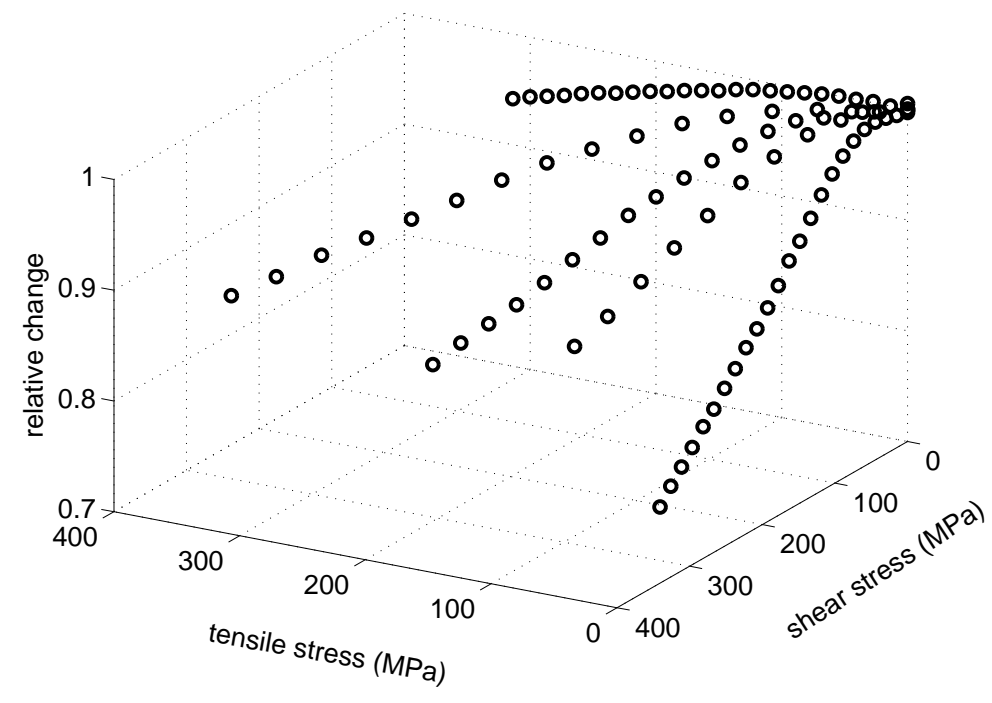

Figure C.1: The influence of stress on the sensor output voltage.

fraction can be performed. To have the data for correlation, a set of samples have been deformed to give different voltage readings and then by metallographic inspection the actual martensite amounts have been determined. The data gathered from these tests and the function used for correlation are plotted in Figure C.2.

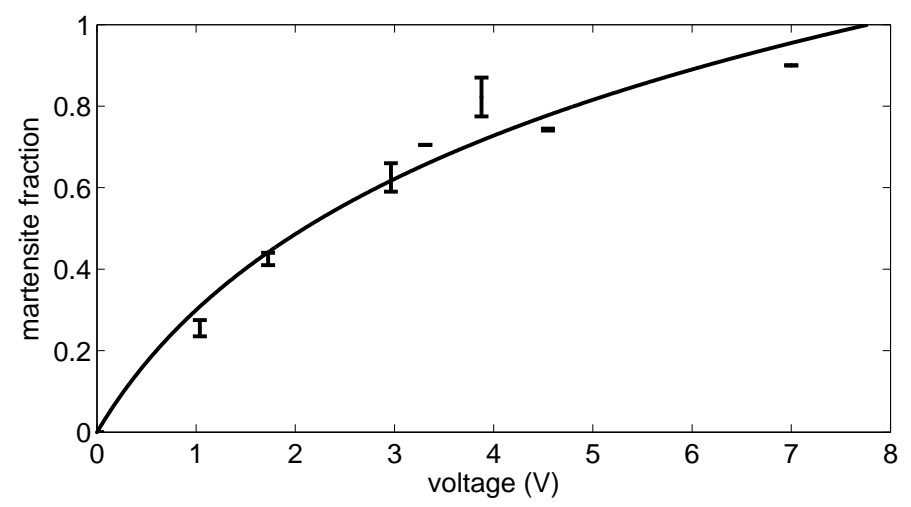

Figure C.2: Correlation of martensite fraction to sensor output. 


\section{Nomenclature}

\section{Greek symbols}

\begin{tabular}{ll}
$\alpha^{\prime}$ & Martensite \\
$\Delta$ & difference; increment \\
$\varepsilon$ & small strain tensor \\
$\varepsilon^{\mathrm{p}}$ & equivalent plastic strain \\
$\varepsilon^{*}$ & eigenstrain (transformation or stress-free strain) \\
$\varepsilon^{\infty}$ & far-field strain \\
$\tilde{\varepsilon}$ & disturbance strain \\
$\varepsilon^{\mathrm{tp}}$ & transformation strain tensor \\
$\varepsilon^{\mathrm{eq}}$ & equivalent strain \\
$\boldsymbol{\Phi}$ & transformation tensor \\
$\gamma$ & Austenite \\
$\kappa$ & bulk modulus \\
$\lambda_{j}$ & $j^{\text {th }}$ eigenvalue of the transformation deformation tensor \\
$\dot{\lambda}$ & plastic flow rate \\
$\mu$ & shear modulus \\
$\nu$ & Poisson's ratio \\
$\phi$ & yield function (plasticity); interpolation function (homogenization) \\
$\boldsymbol{\sigma}$ & Cauchy stress tensor \\
$\nabla$ & Jaumann rate of the Cauchy stress tensor \\
$\odot^{\sigma}$ & Truesdell rate of the Cauchy stress tensor \\
$\boldsymbol{\sigma}^{\prime}$ & deviatoric part of the Cauchy stress tensor \\
$\sigma^{\mathrm{y}}$ & yield stress \\
$\sigma^{\text {eq }}$ & equivalent stress \\
$\sigma^{\mathrm{f}}$ & flow stress \\
$\sigma_{y y}$ & normal component of the Cauchy stress tensor in the $y$ direction \\
$\sigma_{j}^{*}$ & $j^{\text {th }}$ \\
$\tau_{x y}$ & shear component of the Cauchy stress tensor in the $x y$ direction \\
$\xi^{i}$ & local volume fraction of a Martensitic variant \\
& \\
\hline &
\end{tabular}




\section{Roman symbols}

\begin{tabular}{|c|c|}
\hline$a$ & lattice parameter; aspect ratio \\
\hline $\mathcal{A}$ & strain concentration tensor with respect to composite strain \\
\hline$\hat{\mathcal{A}}$ & strain concentration tensor with respect to matrix strain \\
\hline $\mathcal{B}$ & stress concentration tensor with respect to composite stress \\
\hline$\hat{\mathcal{B}}$ & stress concentration tensor with respect to matrix stress \\
\hline $\mathcal{C}^{\mathrm{el}}$ & elasticity tensor \\
\hline $\mathcal{C}^{\mathrm{J}}$ & Continuum tangent operator, in Jaumann terms \\
\hline $\mathcal{C}^{\mathcal{T}}$ & Continuum tangent operator, in Truesdell terms \\
\hline $\mathcal{C}^{\mathrm{J}, \mathrm{el}}$ & elasticity tensor, in Jaumann terms \\
\hline $\mathcal{C}^{\mathrm{J}, \text { alg }}$ & algorithmic tangent operator, in Jaumann terms \\
\hline $\mathcal{C}^{\mathrm{sec}}$ & secant modulus \\
\hline D & deformation rate tensor \\
\hline$D_{y y}$ & normal component of deformation rate tensor in the $y$ direction \\
\hline$D_{x y}$ & shear component of deformation rate tensor in the $x y$ direction \\
\hline $\mathbf{D}^{\operatorname{tr}}$ & transformation deformation rate tensor \\
\hline $\mathbf{D}^{\mathrm{ep}}$ & elastic-plastic deformation rate tensor \\
\hline $\mathbf{E}$ & Green-Lagrange strain tensor \\
\hline $\mathcal{E}$ & Eshelby's tensor \\
\hline$E$ & elastic modulus \\
\hline $\mathbf{e}_{j}$ & $j^{\text {th }}$ eigenvector of the Cauchy stress tensor \\
\hline $\mathbf{F}$ & deformation gradient tensor \\
\hline$f^{\alpha^{\prime}}$ & Martensite volume fraction \\
\hline$f^{i}$ & volume fraction of a Martensitic variant \\
\hline$\Delta G^{\gamma \rightarrow \alpha^{\prime}}$ & Gibbs Free Energy difference of Austenite and Martensite \\
\hline$\Delta G^{\mathrm{cr}}$ & critical Gibbs Free Energy difference \\
\hline $\mathcal{H}$ & strain concentration tensor in the Eshelby theory \\
\hline $\mathbf{I}$ & second-order unit tensor \\
\hline $\mathcal{I}$ & fourth-order symmetric unit tensor \\
\hline $\mathcal{I}^{\mathrm{vol}}$ & volumetric (spherical) part of the fourth-order symmetric unit tensor \\
\hline $\mathcal{I}^{\text {dev }}$ & deviatoric part of the fourth-order symmetric unit tensor \\
\hline$J$ & determinant of the deformation gradient tensor (volume change) \\
\hline $\mathbf{L}$ & velocity gradient tensor \\
\hline$M_{\mathrm{S}}$ & Martensite start temperature \\
\hline$M_{\mathrm{s}}^{\sigma}$ & maximum attainable stress-assisted Martensite start temperature \\
\hline$\stackrel{\dot{\mathrm{d}}}{M_{\mathrm{S}}}$ & maximum attainable strain-induced Martensite start temperature \\
\hline $\mathbf{N}$ & deviatoric stress direction \\
\hline$N_{j}^{*}$ & $j^{\text {th }}$ eigenvalue of the deviatoric principal stress direction \\
\hline n & normal vector \\
\hline $\mathbf{P}$ & nominal (transpose of the $1^{\text {st }}$ Piola-Kirchoff) stress tensor \\
\hline$P^{\text {int }}$ & internal power \\
\hline $\mathbf{R}, \mathbf{Q}$ & rotation tensor \\
\hline$r, m, \Delta G^{\text {off }}$ & parameters of the transformation function \\
\hline $\mathbf{r}$ & plastic flow direction \\
\hline $\mathbf{s}$ & shear direction vector \\
\hline
\end{tabular}


T symmetric transformation deformation tensor

$T$ magnitude of deviatoric transformation deformation; temperature

$T^{\max } \quad$ maximum attainable $T$ for a given stress direction

t traction on a surface

U stretch tensor

$\mathbf{U}_{\mathrm{d}} \quad$ stretch tensor in principle coordinates

$U \quad$ mechanical driving force

$U^{\max } \quad$ maximum resolved mechanical driving force

$V \quad$ volume

W spin tensor

w vector of weight functions

$\mathrm{x} \quad$ current cartesian coordinates

X reference cartesian coordinates

\section{Operators}

$\langle.\rangle_{\omega} \quad$ average over the volume $\omega$

|.| norm

$\mathbf{x}^{\mathrm{T}} \quad$ transpose of $\mathbf{x}$

$\operatorname{det} \mathbf{x}$ determinant of $\mathbf{x}$

$\dot{x} \quad$ time derivative of $\mathrm{x}$

$\nabla x \quad$ gradient of $\mathrm{x}$

\section{General superscripts and subscripts}
$(.)^{i}$
$i^{\text {th }}$ martensitic variant
$(.)^{\mathrm{eq}}$
equivalent
$(.)^{\mathrm{e}}$
elastic part
$(.)^{\mathrm{p}}$
plastic part
$(.)_{0}$
matrix phase (Austenite)
$(.)_{1}$
$(.)^{\mathrm{tr}}$
inclusion phase (Martensite)
$(.)^{\mathrm{tp}}$
transformation
transformation plasticity 



\section{Acknowledgments}

A little more than 4 years ago, I was a PhD student in Turkey and I had decided to make some changes in my life. I would have never imagined that the changes would be this extraordinary. I owe a lot to some people without whom these changes would still be dreams. Now, having almost finished the PhD study in the Netherlands, it is an opportunity for me to remember the people who helped me get here and finish the work that I have started.

I have to admit that as a materials scientist, I had almost no knowledge on mechanics until I started the course 'introduction to continuum mechanics', by Prof. dr. ing. Erman Tekkaya in the Middle East Technical University, in Turkey. Without his lectures, it would be impossible for me to do the work that has been presented in this thesis. Having completed the course, I had the enthusiasm to combine the mechanics knowledge and materials science. Just at that time, a dear friend of mine, Müge Erinç, told me of a $\mathrm{PhD}$ position in the Netherlands which was the first step to make the biggest change in my life. I am very thankful to Müge for letting me know of this position. However, I believe that without the nice comments of Prof. Erman Tekkaya it would still be hard for me to be accepted in the University of Twente. I am more than grateful to him for trusting me.

I want to thank Prof. Han Huétink for giving me the chance to work on this project by accepting me in the group. Now that I was accepted to the group, a very challenging work was ahead of me. I had loads of mechanics and plasticity to learn just to be able to understand at least the presentations of the other $\mathrm{PhD}$ students in the group meetings. For that I would like to thank all the members of the DiekA group, for sharing all their knowledge without any boundaries. Especially, Bert Geijselaers, Ton van den Boogaard and Timo Meinders for keeping their doors open at all times. I am deeply grateful to my supervisor Bert Geijselaers, for his enthusiasm on the project and the very long brain-storming sessions we had in his office. It was always very comforting to know that every problem can be solved, especially when he was around. I want to thank Prof. Han Huétink again for helping us when we were lost in some complex tensorial formulations.

I would like to thank Prof. Han Huétink, Bert Geijselaers, Ton Bor, Wouter Quak and Maarten van Riel for carefully reading my thesis and providing very valuable feedback that shaped the thesis. I greatly acknowledge Akke Suiker and Marc Geers 
for reading the thesis thoroughly and sharing their comments that put the thesis in its final shape. Additionally, the efforts of Katrina Emmett to correct the English language are appreciated.

Apart from the scientific work, I have to acknowledge the financial support of 'Stichting voor Fundamenteel Onderzoek der Materie (FOM)' since it was, on paper, my employer. It was unfortunate that I could not have more contact with FOM, but I am thankful to Maria Teuwissen for dealing with the personnel issues. Back in Twente however, life would be very difficult if Tanja Gerrits and Debbie Zimmermann were not there anytime we needed them. I want to thank them for solving all the difficult administrative deadlocks.

I realize now that actually I was very lucky to be a part of the Applied Mechanics group because of the wonderful social environment. Beginning from the first day I started in the group it felt like home due to the warm friendship of Igor Burchitz, Pawel Owczarek, Maarten van Riel and Marieke Hannink. In time, as newer members such as Ashraf Hadoush, Wouter Quak, Wissam Assaad, Emre Dikmen and of course Didem Akçay Perdahcıoğlu joined the group, it became more and more fun.

Finally, I want to thank my best friend, my wife, Didem Akçay Perdahcıoğlu for her never-ending support and trust in me. Although it rains still as much, the sun seems to shine brighter, now that she is here.

E.S. Perdahcioğlu

Enschede, November 2008 


\section{References}

[1] T. Angel. Formation of martensite in austenitic stainless steels. Journal of the Iron and Steel Institute, 1977:165-174, 1954.

[2] T. Belytschko, W.K. Liu, and B. Moran. Nonlinear Finite Elements for Continua and Structures. John Wiley \& Sons Ltd., 2000.

[3] Y. Benveniste. A new approach to the application of Mori-Tanaka's theory in composite materials. Mechanics of Materials, 6:147-157, 1987.

[4] M. Berveiller and A. Zaoui. An extension of the self-consistent scheme to plastically-flowing polycrystals. Journal of Mechanics and Physics of Solids, 26:325-344, 1979.

[5] H.K.D.H. Bhadeshia. Driving force for martensitic transformation in steels. Metal Science, 15:175-177, 1981.

[6] H.K.D.H. Bhadeshia. Geometry of Crystals. The Institute of Metals, 1987.

[7] A. Bhattacharyya and G. J. Weng. An energy criterion for the stress-induced martensitic transformation in a ductile system. Journal of the Mechanics and Physics of Solids, 42(11):1699-1724, 1994.

[8] G.F. Bolling and R.H. Richman. The plastic deformation of Ferromagnetic FaceCentered Cubic Fe-Ni-C alloys. Philosophical Magazine, 19(158):247-264, 1969.

[9] J. Bonet and R. D. Wood. Nonlinear continuum mechanics for finite element analysis. Cambridge University Press, 1997.

[10] J.S. Bowles and J.K. Mackenzie. The crystallography of martensite transformations i. Acta Metallurgica, 2:129-137, 1954.

[11] J.S. Bowles and J.K. Mackenzie. The crystallography of martensite transformations ii. Acta Metallurgica, 2:138-147, 1954.

[12] J.S. Bowles and J.K. Mackenzie. The crystallography of martensite transformations iii. face-centered cubic to body-centered tetragonal transformations. Acta Metallurgica, 2:224-234, 1954. 
[13] S. Chatterjee and H.K.D.H. Bhadeshia. Transformation induced plasticity assisted steels: stress or strain affected martensitic transformation? Materials Science and Technology, 23(9):1101-1104, 2007.

[14] R.D. Cook. Finite Element Modeling for Stress Analysis. John Wiley \& Sons Inc, 1995.

[15] M. Coret, S. Calloch, and A. Combescure. Experimental study of the phase transformation plasticity of 16MND5 low carbon steel induced by proportional and nonproportional biaxial loading paths. European Journal of Mechanics A, 23:823-842, 2004.

[16] I. Doghri. Mechanics of Deformable Solids. Springer, 2000.

[17] I. Doghri and C. Friebel. Effective elasto-plastic properties of inclusion-reinforced composites. study of shape, orientation and cyclic response. Mechanics of Materials, 37:45-68, 2005.

[18] I. Doghri and A. Ouaar. Homogenization of two-phase elasto-plastic composite materials and structures study of tangent operators, cyclic plasticity and numerical algorithms. International Journal of Solids and Structures, 40:1681$1712,2003$.

[19] I. Doghri and L. Tinel. Micromechanical modeling and computation of elastoplastic materials reinforced with distributed-orientation fibers. International Journal of Plasticity, 21:1919-1940, 2005.

[20] J.D. Eshelby. The determination of the elastic field of an ellipsoidal inclusion, and related problems. Proceedings of the Royal Society of London. Series A, 241(1226):376-396, 1957.

[21] J.D. Eshelby. The elastic fields outside an ellipsoidal inclusion. Proceedings of the Royal Society of London, 252(1271):561-569, 1959.

[22] J.F. Ganghoffer and K. Simonsson. A micromechanical model of the martensitic transformation. Mechanics of Materials, 27:125-144, 1998.

[23] A.C. Gavazzi and D.C. Lagoudas. On the numerical evaluation of eshelby's tensor and its application to elastoplastic fibrous composites. Computational Mechanics, 7:13-19, 1990.

[24] H.J.M. Geijselaers and E.S. Perdahcıŏglu. Mechanically induced martensitic transformation as a stress-driven process. Scripta Materialia, 60(1):29-31, 2009.

[25] C. Gonzáles and J. Llorca. A self-consistent approach to the elasto-plastic behaviour of two-phase materials including damage. Journal of the Mechanics and Physics of Solids, 48:675-692, 2000.

[26] G.W. Greenwood and R.H. Johnson. The deformation of metals under small stresses during phase transformations. Proceedings of the Royal Society, 283A:403-422, 1965. 
[27] H. Hallberg, P. Håkansson, and M. Ristinmaa. A constitutive model for the formation of martensite in austenitic steels under large strain plasticity. International Journal of Plasticity, 23:1213-1239, 2007.

[28] H.N. Han, C.G. Lee, C.-S. Oh, T.-H. Lee, and S.-J. Kim. A model for deformation behavior and mechanically induced martensitic transformation of metastable austenitic steel. Acta Materialia, 52:5203-5214, 2004.

[29] Z. Hashin and S. Shtrikman. A variational approach to the theory of the elastic behavior of multiphase materials. Journal of Mechanics and Physics of Solids, 11:127-140, 1963.

[30] M.T. Heath. Scientific Computing, An Introductory Survey. McGraw-Hill, second edition, 2002.

[31] P. Hilkhuijsen. Determination of dislocation density by x-ray diffraction. Master's thesis, University of Twente, 2007.

[32] R. Hill. Elastic properties of reinforced solids: some theoretical principles. Journal of mechanics and physics of solids, 11:357-372, 1963.

[33] R. Hill. Continuum micro-mechanics of elastoplastic polycrystals. Journal of Mechanics and Physics of Solids, 13:89-101, 1965.

[34] R. Hill. A self-consistent mechanics of composite materials. Journal of mechanics and physics of solids, 13:213-222, 1965.

[35] Homer. The Odyssey, volume IX. The Internet Classics Archive, 800 B.C. English translation by Samuel Butler.

[36] W. Huang. "yield" surfaces of shape memory alloys and their applications. Acta Materialia, 47(9):2769-2776, 1999.

[37] J. Huétink. On the simulation of thermo-mechanical forming processes. $\mathrm{PhD}$ thesis, University of Twente, the Netherlands, 1986.

[38] T.J.R. Hughes. The Finite Element Method. Dover Publications Inc, 1987.

[39] T. Iwakuma and S. Nemat-Nasser. Finite elastic-plastic deformation of polycrystalline metals. Proceedings of the Royal Society in London, A 394:87-119, 1984.

[40] T. Iwamato, T. Tsuta, and Y. Tomita. Investigation on deformation mode dependence of strain-induced martensitic transformation in TRIP steels and modelling of transformation kinetics. International Journal of Mechanical Sciences, 40:173-182, 1998.

[41] P.J. Jacques, Q. Furnémont, F. Lani, T. Pardoen, and F. Delannay. Multiscale mechanics of TRIP-assisted multiphase steels: I. characterization and mechanical testing. Acta Materialia, 55:3681-3693, 2007. 
[42] L. Kaufman and M. Cohen. Thermodynamics and kinetics of martensitic transformations. Progress in Metal Physics, 7:165-245, 1958.

[43] A.S. Khan and S. Huang. Continuum Theory of Plasticity. John Wiley \& Sons Inc, 1995.

[44] V.G. Kouznetsova, W.A.M. Brekelmans, and F.P.T. Baaijens. An approach to micro-macro modeling of heterogeneous materials. Computational Mechanics, $27: 37-48,2001$.

[45] V.G. Kouznetsova, M.G.D. Geers, and W.A.M. Brekelmans. Multi-scale secondorder homogenization of multi-phase materials: a nested finite element solution strategy. Computer Methods in Applied Mechanics and Engineering, 193:55255550, 2004.

[46] E. Kröner. Berechnung der elastischen Konstanten des Vielkristalls aus den Konstanten des Einkristalls. Zeitschrift für Physik, 151:504-518, 1958.

[47] S. Kundu and H.K.D.H. Bhadeshia. Transformation texture in deformed stainless steel. Scripta Materialia, 55:779-781, 2006.

[48] S. Kundu and H.K.D.H. Bhadeshia. Crystallographic texture and intervening transformations. Scripta Materialia, 57:869-872, 2007.

[49] R. Laarhoven. Martensitic transformation and springback of austenitic nanoflex in pure bending. Internal Report MT 08.03, Faculty of Mechanical Engineering, Eindhoven University of Technology, 2007.

[50] A.A. Lebedev and V.V. Kosarchuk. Influence of phase transformations on the mechanical properties of austenitic stainless steels. International Journal of Plasticity, 16:749-767, 2000.

[51] V.I. Levitas, A.V. Idesman, and G.B. Olson. Continuum modeling of straininduced martensitic transformation at shear-band intersections. Acta Materialia, 47:219-233, 1999.

[52] C. Lexcellent and A. Schlömerkemper. Comparison of several models for the determination of the phase transformation yield surface in shape-memory alloys with experimental data. Acta Materialia, 55:2995-3006, 2007.

[53] M. Lin, G.B. Olson, and M. Cohen. Distributed-activation kinetics of heterogeneous martensitic nucleation. Metallurgical Transactions A, 23A:29872998, 1992.

[54] J. Lof and A.H. van den Boogaard. Adaptive return mapping algorithms for J2 elasto-viscoplastic flow. International Journal For Numerical Methods In Engineering, 51:1283-1298, 2001.

[55] V.A. Lubarda. Elastoplasticity theory. CRC Press, 2002. 
[56] M. Maalekian, E. Kozeschnik, S. Chatterjee, and H.K.D.H. Bhadeshia. Mechanical stabilisation of eutectoid steel. Materials Science and Technology, 23(5):610-612, 2007.

[57] C.L. Magee. Transformation kinetics, microplasticity and aging of Martensite in Fe-31 Ni. PhD thesis, Carnegie Institute of Technology, 1966.

[58] C.L. Magee and H.W. Paxton. The microplastic response of partially transformed Fe-31Ni. Transaction of the metallurgical society of AIME, 242:1741-1749, 1968.

[59] F. Marketz and F.D. Fischer. A micromechanical study on the coupling effect between microplastic deformation and martensitic transformation. Computational Materials Science, 3:307-325, 1994.

[60] D. San Martin, T. Nulens, L.C.N. Louws, V.G. Kouznetsova, M.P.H.F.L. van Maris, M.G.D. Geers, and S. van der Zwaag. In-situ study of martensite formation in metastable austenitic steel. Materials Characterization, 2008. In preparation.

[61] T. Mura. Micromechanics of defects in solids. Martinus Nijhoff Publishers, 1982.

[62] J.C. Nagtegaal, D.M. Parks, and J.C. Rice. On numerical accurate finite element solutions in the fully plastic range. Computer Methods in Applied Mechanics and Engineering, 4:153-177, 1974.

[63] S. Nemat-Nasser. Averaging theorems in finite deformation plasticity. Mechanics of Materials, 31:493-523, 1999.

[64] S. Nemat-Nasser and T. Iwakuma. Elastic-plastic composites at finite strains. International Journal of Solids and Structures, 21(1):55-65, 1985.

[65] H. Nolles, J. Post, and J. Beyer. Inductive measurements of the stress assisted and strain induced martensite transformations of Sandvik maraging steel 1RK91 before, during and after metal forming,. Journal de Physique IV, 112:425-428, 2003.

[66] G.B. Olson. Martensite, chapter 1 - Introduction: Martensite in Perspective. ASM, 1992. Edited by G.B. Olson and W.S. Owen.

[67] G.B. Olson and M. Cohen. A mechanism for the strain-induced nucleation of martensitic transformations. Journal of the Less-Common metals, 28:107-118, 1972 .

[68] G.B. Olson and M. Cohen. Kinetics of strain-induced martensitic nucleation. Metallurgical Transactions A, 6A:791-795, 1975.

[69] G.B. Olson and M. Cohen. Martensitic transformation as a deformation process. In S.D. Antovich, R.O Ritchie, and W.W. Gerberich, editors, Proceedings of Mechanical Properties and Phase Transformations in Engineering Materials, pages 367-389, 1986. 
[70] J.R. Patel and M. Cohen. Criterion for the action of applied stress in the martensitic transformation. Acta Metallurgica, 1:531-538, 1953.

[71] E.S. Perdahcioğlu. Biaxial tests on Sandvik Nanoflex. Technical Report P06.1.076, Netherlands Institute for Metals Research, 2006.

[72] B. Petit, N. Gey, M. Cherkaoui, B. Bolle, and M. Humbert. Deformation behavior and microstructure/texture evolution of an annealed 304 AISI stainless steel sheet. experimental and micromechanical modeling. International Journal of Plasticity, 23(2):323-341, 2007.

[73] O. Pierard. Micromechanics of inclusion-reinforced composites in elasto-plasticity and elasto-viscoplasticity: modeling and computation. $\mathrm{PhD}$ thesis, Université Catholique de Louvain, 2006.

[74] J. Post. On the constitutive behaviour of Sandvik Nanoflex. PhD thesis, University of Twente, 2004.

[75] V. Raghavan. Martensite, chapter 11 - Kinetics of Martensitic Transformations. ASM, 1992. Edited by G.B. Olson and W.S. Owen.

[76] A.C.E. Reid and G.B. Olson. A continuum description of dislocations in elastically nonlinear media: martensitic embryo formation. Materials Science and Engineering A, 309-310:370-376, 2001.

[77] J. Serri, M. Martiny, and G. Ferron. Finite element analysis of the effects of martensitic phase transformation in TRIP steel sheet forming. International Journal of Mechanical Sciences, 47:884-901, 2005.

[78] J.C. Simo and T.J.R. Hughes. Computational Inelasticity. Springer, 1998.

[79] C.S. Smith. Martensite, chapter 3 - A history of Martensite: Early ideas on the structure of steel. ASM, 1992. Edited by G.B. Olson and W.S. Owen.

[80] R.G. Stringfellow, D.M. Parks, and G.B. Olson. A constitutive model for transformation plasticity accompanying strain-induced martensitic transformation in metastable austenitic stainless steels. Acta Metallurgica and Materialia, 40(7):1703-1716, 1992.

[81] H. Takuda, K. Mori, T. Masachika, E. Yamazaki, and Y. Watanabe. Finite element analysis of the formability of an austenitic stainless steel sheet in warm deep drawing. Journal of Materials Processing Technology, 143-144:242-248, 2003.

[82] I. Tamura. Deformation-induced martensitic transformation and transformationinduced plasticity in steels. Metal Science, 16(5):245-253, 1982.

[83] K. Tanaka and T. Mori. The hardening of crystals by non-deforming particles and fibres. Acta Metallurgica, 18:931-941, 1970. 
[84] G.P. Tandon and G.J. Weng. Average stress in the matrix and effective moduli of randomly oriented composites. Composites Science and Technology, 27:11-132, 1986.

[85] D.D. Tjahjanto, A.S.J. Suiker, S. Turteltaub, P.E.J. Rivera Diaz del Castillo, and S. van der Zwaag. Micromechanical predictions of TRIP steel behavior as a function of microstructural parameters. Computational Materials Science, 41(3):107-116, 2007.

[86] Y. Tomita and T. Iwamato. Constitutive modeling of TRIP steel and its application to the improvement of mechanical properties. International Journal of Mechanical Sciences, 37:1295-1305, 1995.

[87] Z. Tourki, H. Bargui, and H. Sidhom. The kinetic of induced martensitic transformation its effect on forming limit curves in the AISI 304 stainless steel. Journal of Materials Processing Technology, 166:330-336, 2005.

[88] J.C. Videau, G. Cailletaud, and A. Pinau. Experimental study of the transformation-induced plasticity in a Cr-Ni-Mo-Al-Ti steel. Journal de Physique IV, 6:465-474, 1996.

[89] C.M. Wayman. Introduction to the crystallography of martensitic transformations. The Macmillan Company, 1964.

[90] M.S. Wechsler, D.S. Lieberman, and T.A. Read. On the theory of the formation of martensite. Transactions AIME, 197:1503-1515, 1953.

[91] Y.H. Yan, G.Y. Kai, and M.D. Jian. Transformation behavior of retained austenite under different deformation modes for low alloyed TRIP-assisted steels. Materials Science and Engineering A, 441:331-335, 2006.

[92] O.C. Zienkiewicz and R.L. Taylor. The Finite Element Method, volume 1,2,3. Butterworth-Heinemann, fifth edition, 2000. 